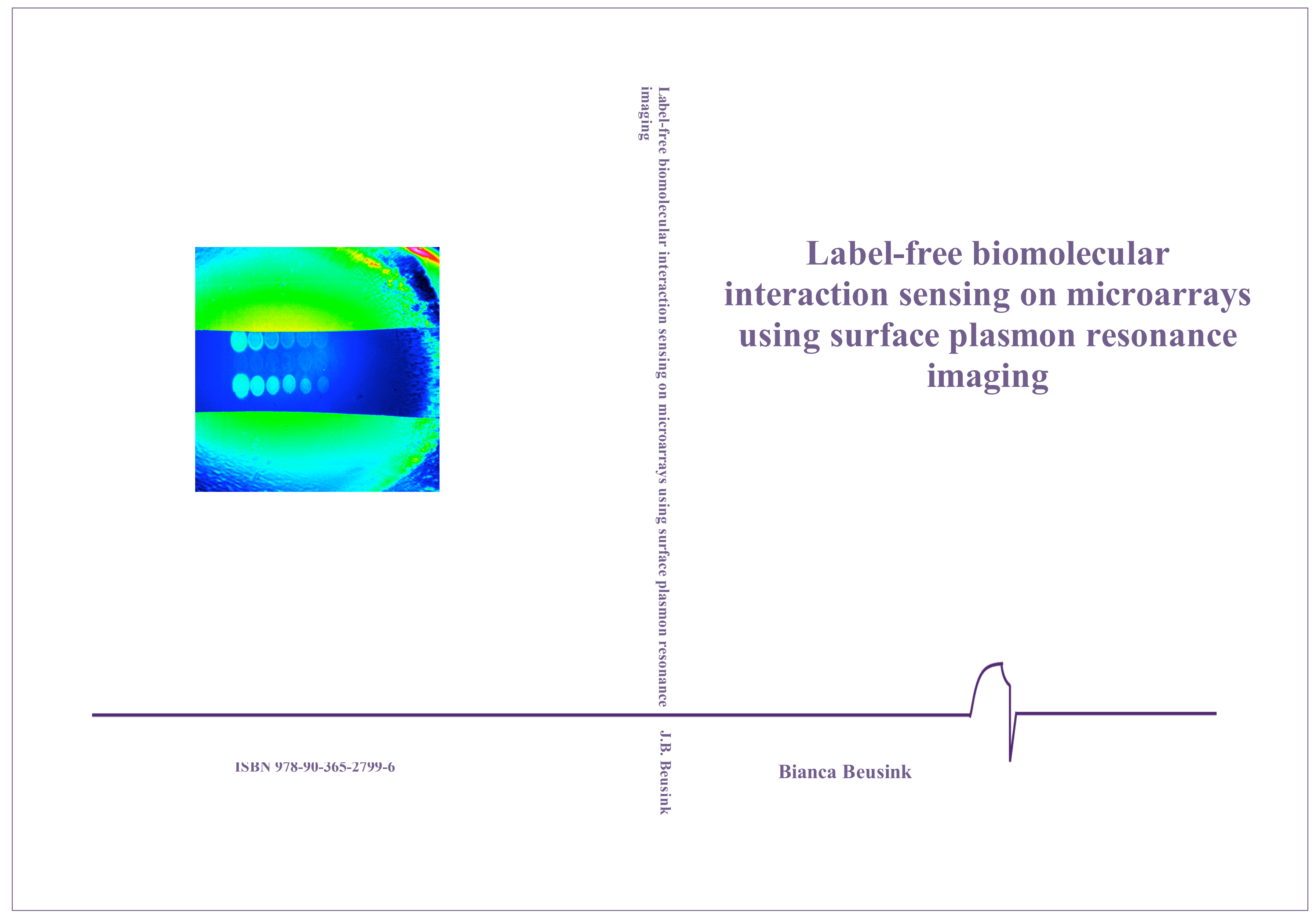


I have the pleasure to invite you to atten

the public defense

of my doctoral thesis

entitled

\section{LABEL-FREE BIOMOLECULAR \\ INTERACTION SENSING ON \\ MICROARRAYS USING \\ SURFACE PLASMON \\ RESOSNANCE IMAGING}

on Thursday, 26 February 2009

$$
\text { at } 13.15 \mathrm{hrs}
$$

in room 2 of the Spiegel

at the University of Twente

After the defense

There will be a reception

in the cafeteria

Paranymphs:

Mink Hoexum

Juliane Teapal

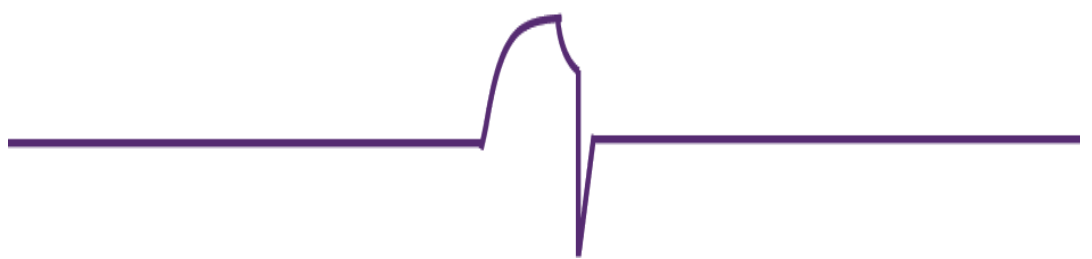

Bianca Beusink

Ziegelhäuser Landstraße 45

69120 Heidelberg, BW

$$
\text { Germany }
$$

biancabeusink@gmail.com

+31 6-5493 8283 (mobile)

+49 176-6194 4080 (mobile)

+49 6221-326 9004 (home) 


\section{LABEL-FREE BIOMOLECULAR INTERACTION SENSING ON MICROARRAYS USING SURFACE PLASMON RESONANCE IMAGING}

Bianca Beusink 
The research described in this thesis was carried out at the BIOS Lab-on-a-Chip group of the $\mathrm{MESA}^{+}$Institute for Nanotechnology of the University of Twente, Enschede, The Netherlands. This research was financially supported by the Dutch Technology foundation STW project TMM 06209.

Members of the committee:

Chairman

Prof. Dr. G. van der Steenhoven

University of Twente

Promotor

Prof. Dr. Ir. A. van den Berg

University of Twente

Assistant Promotor

Dr. Ir. R.B.M. Schasfoort

University of Twente

Dr. E.T. Carlen

University of Twente

Members

Prof. Dr. V. Subramaniam

University of Twente

Prof. Dr. L.W.M.M. Terstappen

University of Twente

Prof. Dr. G.J.M. Pruijn

Radboud University Nijmegen

Dr. M.T. McDermott

University of Alberta

Beusink, Judith Bianca

Label-free biomolecular interaction sensing on microarrays using surface plasmon resonance imaging

PhD thesis University of Twente, Enschede, The Netherlands

ISBN: 978-90-365-2799-6

Publisher: Wöhrman Print Service, Zutphen, The Netherlands

Cover design: "Bianca" (white) cover with the "heartbeat" of SPR on the front and SPRi result on the back.

Copyright (C 2009 by Judith Bianca Beusink, Enschede, The Netherlands 


\title{
LABEL-FREE BIOMOLECULAR INTERACTION SENSING ON MICROARRAYS USING SURFACE PLASMON RESONANCE IMAGING
}

\author{
Dissertation \\ to obtain \\ the degree of doctor at the University of Twente \\ on the authority of the rector magnificus, \\ Prof. Dr. H. Brinksma, \\ on account of the decision of the graduation committee, \\ to be publicly defended \\ on Thursday, the $26^{\text {th }}$ of February 2009 at 13.15 hrs
}

By

Judith Bianca Beusink

born on $10^{\text {th }}$ December 1975

in Holten, The Netherlands. 
This dissertation has been approved by

Promotor: Prof. Dr. Ir. Albert van den Berg

Assistant promotor: Dr. Ir. Richard B.M. Schasfoort

Assistant promotor: Dr. Edwin T. Carlen 
To the beauty of all the little things around us.... 



\section{Table of Contents}

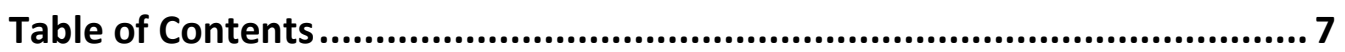

1 Aim \& thesis outline .......................................................... 1

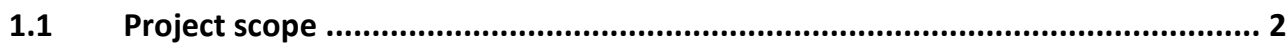

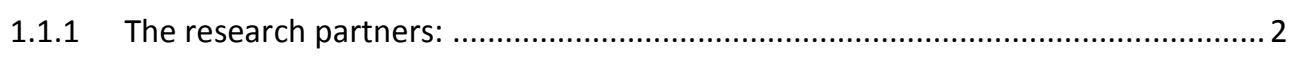

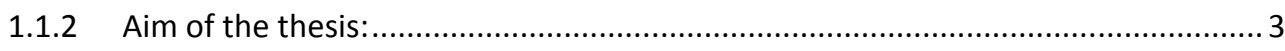

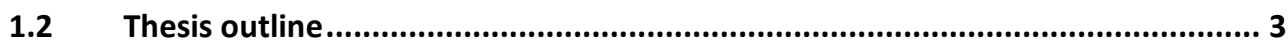

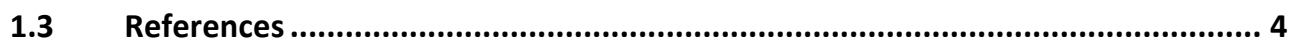

2 Introduction ...................................................................... 7

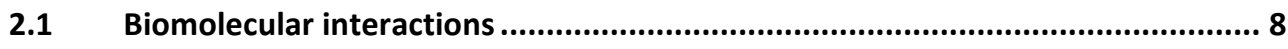

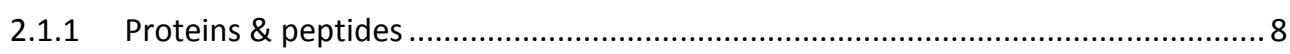

2.1.2 Antibodies \& autoimmune diseases ........................................................... 10

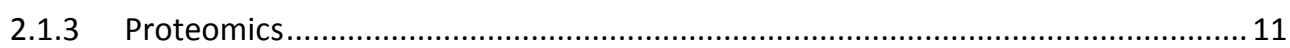

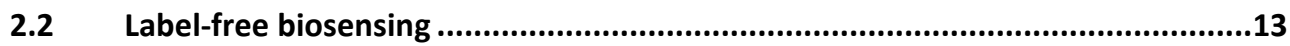

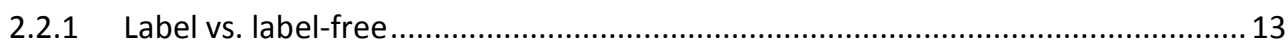

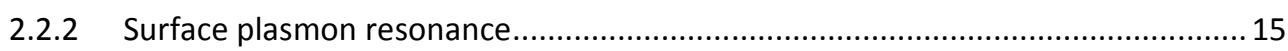

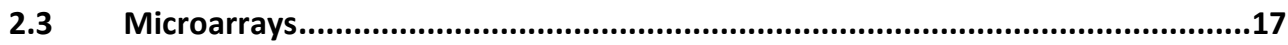

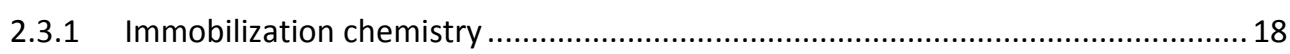

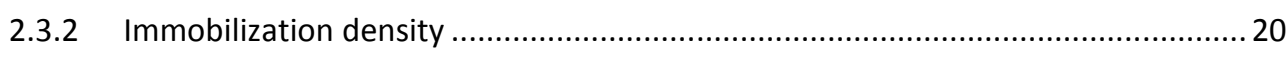

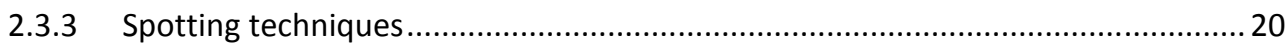




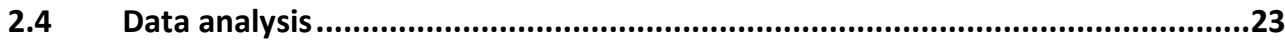

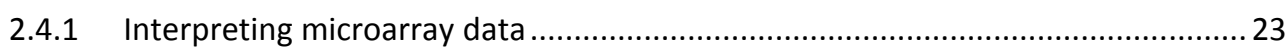

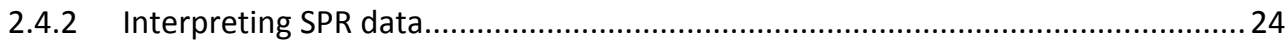

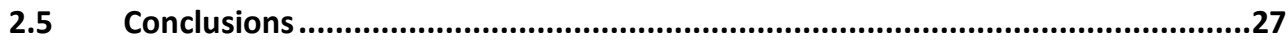

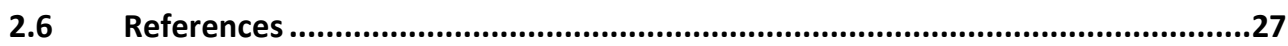

3 A soft-lithography based ligand immobilization technique ................. 35

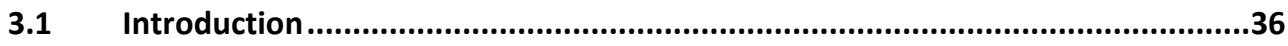

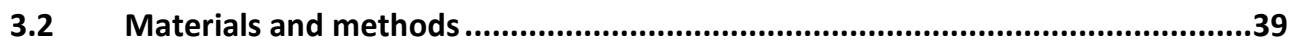

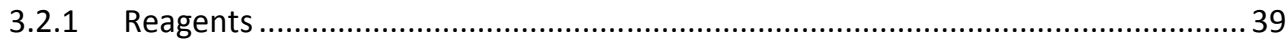

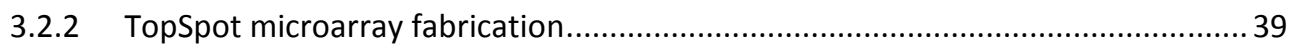

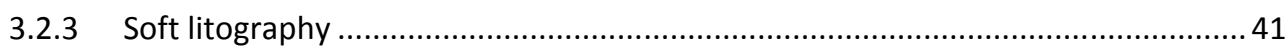

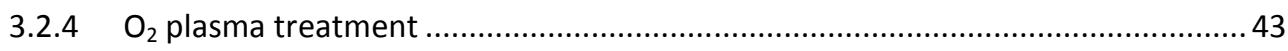

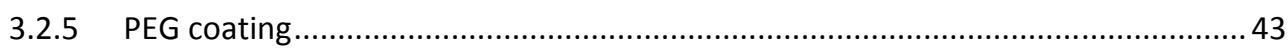

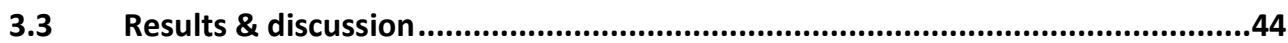

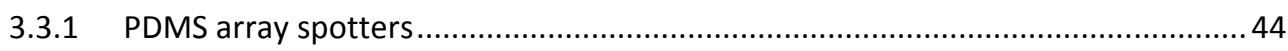

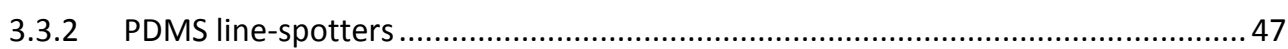

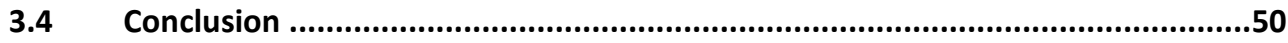

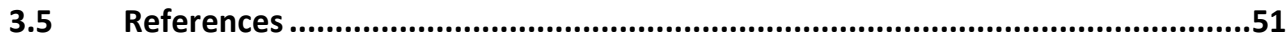

4 Angle-scanning SPR imaging for detection of biomolecular interactions on microarrays ........................................................... 55

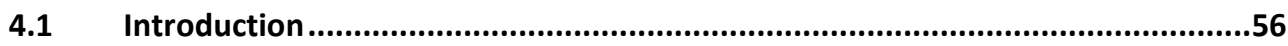

4.2 Materials and methods .................................................................................57

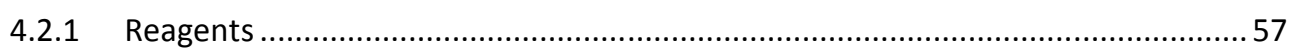

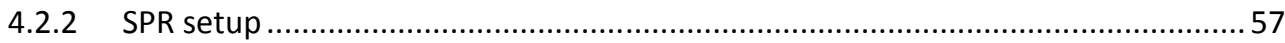




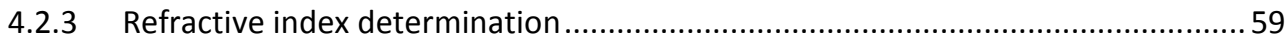

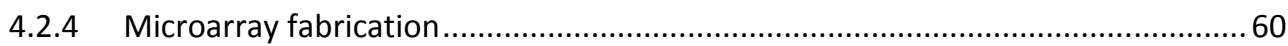

4.2.5 Detection of biomolecular interactions on the peptide/antibody microarray.......60

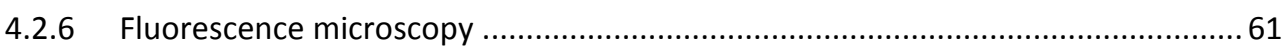

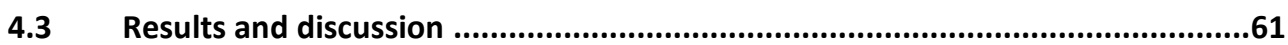

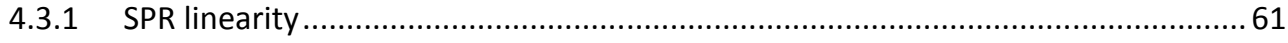

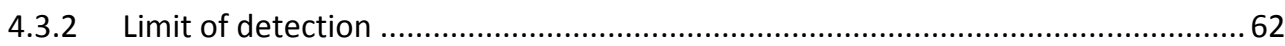

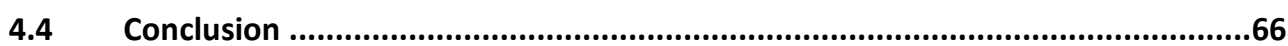

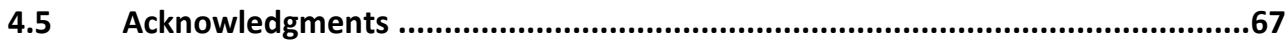

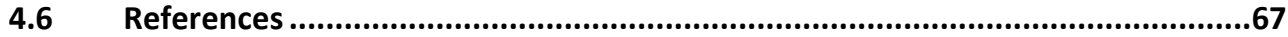

\section{Biomolecular interaction monitoring of autoantibodies by scanning} SPR microarray imaging .......................................................... 71

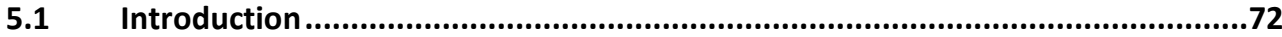

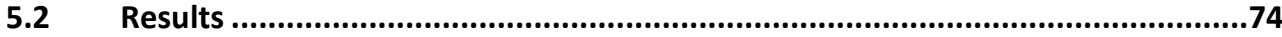

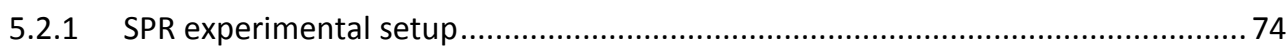

5.2.2 Monitoring the interaction of a peptide array with serum antibodies ................. 76

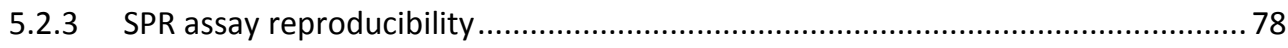

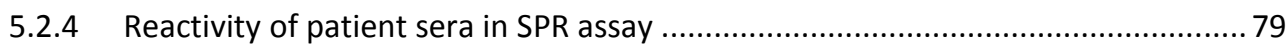

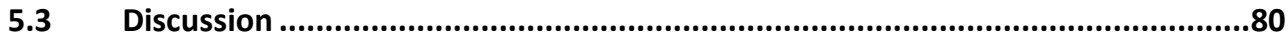

$5.4 \quad$ Experimental procedures .............................................................................82

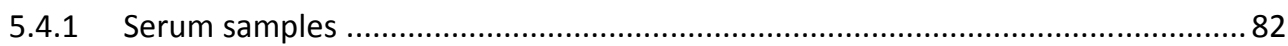

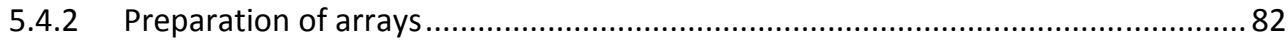

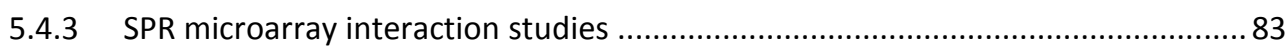

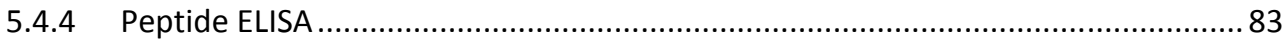

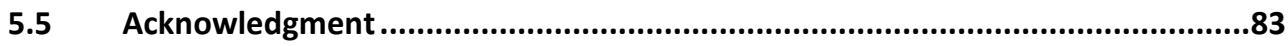


6 Ratio-diluted ligand density assays for single analyte kinetic analysis.. 87

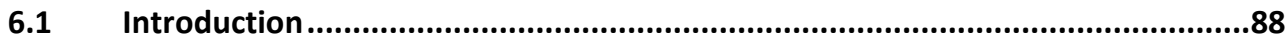

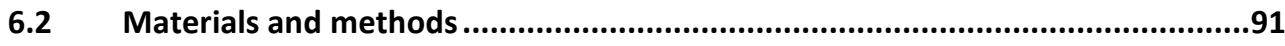

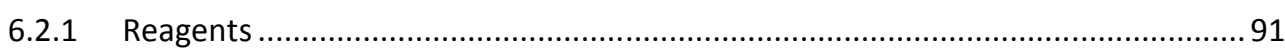

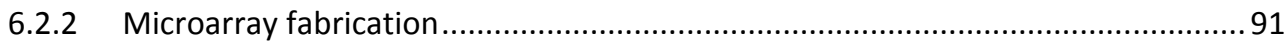

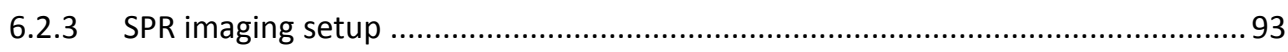

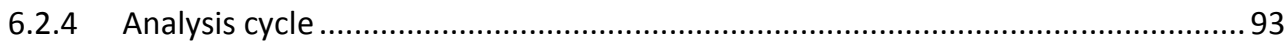

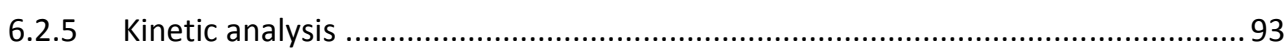

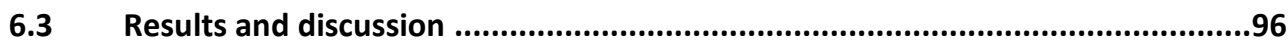

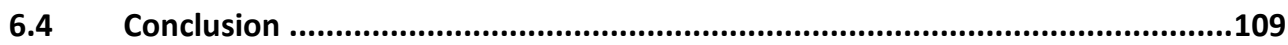

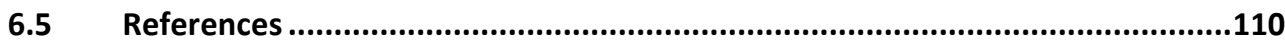

7 Conclusions \& recommendations................................................ 115

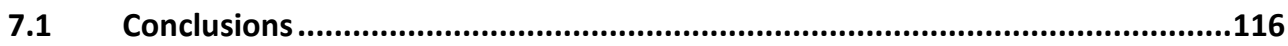

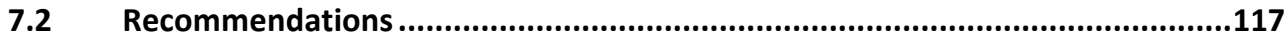

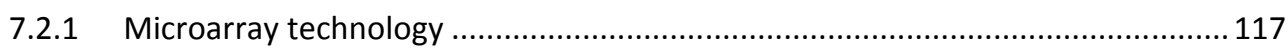

7.2.2 Increased measurement capacity and throughput ....................................... 118

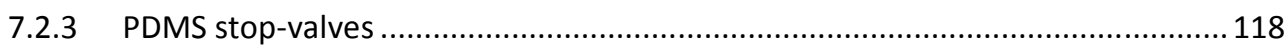

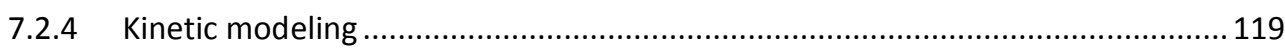

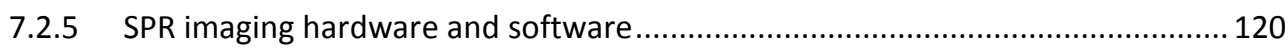

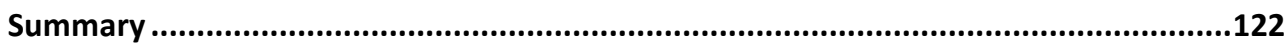

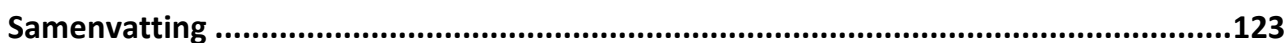

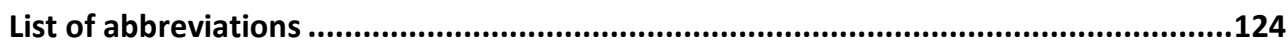


List of publications.......................................................................................................126

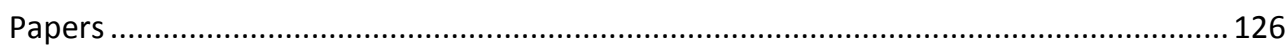

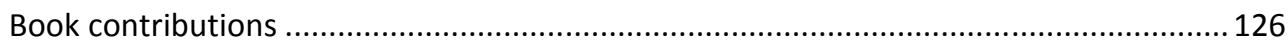

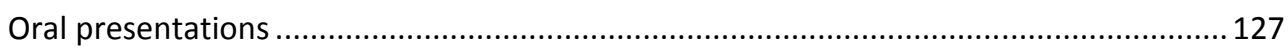

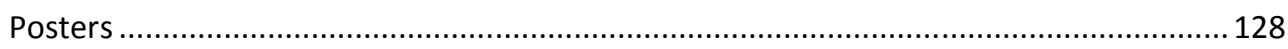

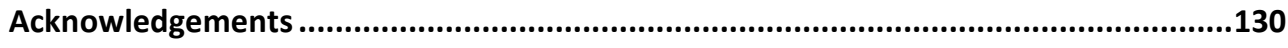





\section{$1 \quad$ Aim \& thesis outline}

This first chapter provides the reader a brief introduction to the thesis "Biomolecular interaction sensing on microarrays using surface plasmon resonance imaging". The aim of the thesis is to develop an orientation controlled peptide microarray to study the binding of autoantibodies using an SPR imaging system. An outline of the thesis is presented here. 


\subsection{Project scope}

Driven by the need for alternative technologies for separation and identification of the highly complex protein mixtures that are inherent to biomarker discovery, drug screening, disease diagnosis and treatment, three Dutch universities (University of Twente, Radboud University Nijmegen and Utrecht University) initiated a project titled "Proteomics on a chip for monitoring autoimmune diseases" which was funded by the Dutch national technology foundation STW in collaboration with the IOP genomics program, TMM 06209. The goal of the project is to develop a Lab-on-a-Chip (LOC) device for detection of autoimmune diseases and new biomarker discovery.

\subsubsection{The research partners:}

- University of Twente:

- Development of a microfluidic free-flow electrophoresis (FFE) chip for the separation of proteins in biological samples e.g. serum,

- Development of new microarray based immobilization techniques and label-free detection of autoantibodies in serum samples with surface plasmon resonance imaging (SPR imaging).

- Radboud University Nijmegen: characterization and production of relevant autoantigens and autoantibodies for the diagnosis and fingerprinting of autoimmune diseases, such as rheumatoid arthritis (RA).

- Utrecht University:

- Investigate capillary electrophoresis (CE) and isoelectric focusing (IEF) technologies for biological sample pretreatment,

- Integration of mass spectrometry (MS) and SPR for the identification of new biomarkers.

An overview of proteomics on a chip can be read in the following publication ${ }^{1}$ and through the output of the research partners 23456789101112 . 


\subsubsection{Aim of the thesis:}

Aims are: 1) the development of orientation controlled ligand immobilization of peptides in a microarray format by commercial piezoactuation deposition, commonly called spotting, and newly developed strategies utilizing microfluidic techniques, 2) the determination of the specificity, sensitivity and detection limit of immunological interactions in an SPR imaging system, 3) study the affinity binding kinetics using a simple pressure driven flow-cell using different methods for varying ligand and analyte concentration.

\subsection{Thesis outline}

A brief summary of the thesis chapters is provided below.

Chapter 2: "Introduction", this chapter contains a short introduction to the motivation and various aspects and techniques regained to understand and measure biomolecular interactions. Some important suggestions are: Why is label-free sensing of biomolecules so important? What are the advantages of using microarrays and surface plasmon resonance imaging?

Chapter 3: "A soft-lithography based ligand immobilization technique", this chapter gives an overview of the various soft-lithography based ligand immobilization techniques, with an emphasis on PDMS based microfluidic spotting devices.

Chapter 4: "Angle-scanning SPR imaging for detection of biomolecular interactions on microarrays", this chapter is an introduction to scanning angle SPR with a comparison to fixed angle SPR. Biotinylated peptides immobilized on the surface were used to study the antibody binding. Furthermore the detection limit is discussed by means of a microarray experiment. ${ }^{13}$.

Chapter 5: "Biomolecular interaction monitoring of autoantibodies by surface plasmon resonance microarray imaging", this chapter demonstrates the diagnostic relevance of monitoring biomolecular interactions by using SPR imaging, with an application to studying rheumatoid arthritis autoantibodies in diluted serum samples. ${ }^{14}$.

Chapter 6: "Ratio-diluted ligand density assays for single analyte kinetic analysis", this chapter describes an alternative method to obtain analyte-ligand kinetic information by 
using a single analyte concentration and ratio-diluted ligand concentrations. This method has the advantage of requiring less analyte sample volume, reduces the surface regeneration steps and decreases the experimental time needed.

Chapter 7: "Conclusions and recommendations", this chapter will give a concluding overview of the material discussed throughout this thesis, and finishes with recommendations on how to improve or carry on with the research.

\subsection{References}

1 Schasfoort, R.B.M., Proteomics-on-a-chip: the challenge to couple lab-on-achip unit operations. Expert Review of Proteomics 1 (1), 123-132 (2004).

2 Silvertand, L.H.H.; Machtejevas, E.; Hendriks, R.; Unger, K.K.; van Bennekom, W.P.; de Jong, G.J., Selective protein removal and desalting using microchip CE. Journal of Chromatography B 839 (1-2), 68 (2006).

3 Silvertand, L.H.H.; Toraño, J. Sastre.; de Jong, G.J.; van Bennekom, W.P., Improved repeatability and matrix-assisted desorption/ionization - time of flight mass spectrometry compatibility in capillary isoelectric focusing. Electrophoresis 29 (10), 1985-1996 (2008).

4 Silvertand, L.H.H., Toraño, J. Sastre, van Bennekom, W.P., and de Jong, G.J., Recent developments in capillary isoelectric focusing. Journal of Chromatography A In Press, Corrected Proof.

5 Visser, N.F.C.; Scholten, A.; van den Heuvel, R.H.H.; Heck, A.J.R., Surfaceplasmon-resonance-based chemical proteomics: Efficient specific extraction and semiquantitative identification of cyclic nucleotide-binding proteins from cellular lysates by using a combination of surface plasmon resonance, sequential elution and liquid chromatography-tandem mass spectrometry. ChemBioChem 8 (3), 298-305 (2007).

6 Visser, N.F.C.; Heck, A.J.R., Surface plasmon resonance mass spectrometry in proteomics. Expert Review of Proteomics 5 (3), 425-433 (2008).

$7 \quad$ Kohlheyer, D.; Besselink, G.A.J.; Lammertink, R.G.H.; Schlautmann, S.; Unnikrishnan, S.; Schasfoort, R.B.M., Electro-osmotically controllable multiflow microreactor. Microfluidics and Nanofluidics 1 (3), 242-248 (2005). 
Kohlheyer, D.; Besselink, G.A.J.; Schlautmann, S.; Schasfoort, R.B.M., Freeflow zone electrophoresis and isoelectric focusing using a microfabricated glass device with ion permeable membranes. Lab on a Chip 6 (3), 374-380 (2006).

9 Kohlheyer, D.; Eijkel, J.C.T.; Schlautmann, S.; van den Berg, A.; Schasfoort, R.B.M., Microfluidic high-resolution free-flow isoelectric focusing. Analytical Chemistry 79 (21), 8190-8198 (2007).

10 Kohlheyer, D.; Eijkel, J.C.T.; Schlautmann, S.; van den Berg, A.; Schasfoort, R.B.M., Bubble-free operation of a microfluidic free-flow electrophoresis chip with integrated Pt electrodes. Analytical Chemistry 80 (11), 4111-4118 (2008).

11 Kohlheyer, D.; Eijkel, J.C.T.; van den Berg, A.; Schasfoort, R.B.M., Miniaturizing free-flow electrophoresis - a critical review. Electrophoresis 29 (5), 977-993 (2008).

12 Kohlheyer, D.; Unnikrishnan, S.; Besselink, G.A.J.; Schlautmann, S.; Schasfoort, R.B.M., A microfluidic device for array patterning by perpendicular electrokinetic focusing. Microfluidics and Nanofluidics 4 (6), 557-564 (2008).

13 Beusink, J.B.; Lokate, A.M.C.; Besselink, G.A.J.; Pruijn, G.J.M.; Schasfoort, R.B.M., Angle-scanning SPR imaging for detection of biomolecular interactions on microarrays. Biosensors and Bioelectronics 23 (6), 839-844 (2008).

14 Lokate, A.M.C.; Beusink, J.B.; Besselink, G.A.J.; Pruijn, G.J.M.; Schasfoort, R.B.M., Biomolecular Interaction Monitoring of Autoantibodies by Scanning Surface Plasmon Resonance Microarray Imaging. Journal of the American Chemical Society 129 (45), 14013-14018 (2007). 



\section{Introduction}

This chapter gives a short introduction of the various aspects and techniques used to measure and study biomolecular interactions. First, a general introduction of biomolecular interactions, including peptides and proteins, antibodies and immune diseases, and proteomics is given. Then, the concept of label-free biosensing is introduced, with special emphasis on SPR. Finally, the principle and role of microarrays and data analysis are described and general conclusions discussed. 


\subsection{Biomolecular interactions}

Biomolecules are the building blocks of life. They primarily consist of carbon and hydrogen, along with nitrogen, oxygen, phosphorus and sulfur. Other elements can also be incorporated, however are much less common. Biomolecules, such as nucleic acids contain the blueprint of an organism, as they remain relatively stable throughout the life time of the organism. Nucleotides are the basis or building blocks of nucleic acids, DNA (deoxyribonucleic acid) and RNA (ribonucleic acid). Nucleic acids are translated into proteins, which are involved in the regulation of the metabolic processes. Knowledge of the genome (the entire genetic collection of an organism) is very important for studying the dynamics of the proteome of an organism ${ }^{1}$. In this thesis the main focus will be on proteins and measuring their interactions.

\subsubsection{Proteins \& peptides}

Proteins and peptides are widely studied and used in biological research ${ }^{2}{ }^{3}$. Current research focuses on protein and peptide structure, modeling, expression, analysis, and their interactions with each other and other molecules and the different fields of application ${ }^{4}$.

Proteins are involved in the maintenance and metabolic processes in living organisms. Proteins and peptides are polymers consisting of 20 different types of $\alpha$-amino acids as their building blocks. The amino acids are covalently linked to each other by the formation of an amide bond between $\alpha$-amino and $\alpha$-carboxyl groups, which is also called a peptide bond, shown in Fig. 2.1.

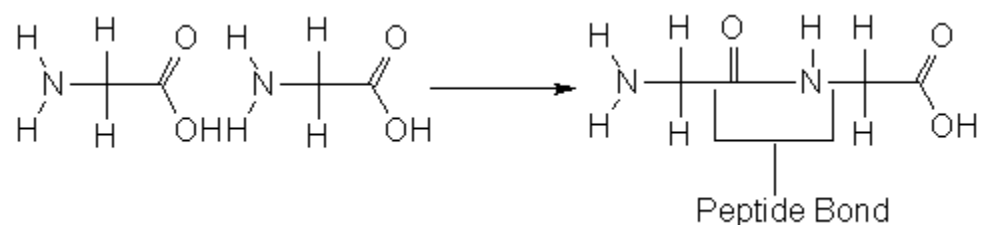

Fig. 2.1. The chemical formula of the peptide bond is shown here ${ }^{3}$. 
Chains containing only a few (2-12) amino acid residues are referred to as oligopeptides, longer chains are called polypeptides, all proteins are polypeptides. Standard polypeptides retain an un-reacted amino group at one end (called the amino terminus or $\mathrm{N}$-terminus) and an un-reacted carboxyl group at the other end (the carboxyl terminus or C-terminus) 3 . Protein molecules have four levels of structural organization. The primary structure of proteins consists of a linear sequence of amino acids and determines the secondary and tertiary structure. The secondary structure is a local folding, of which $\alpha$-helixes and $\beta$ sheets are the most common examples as shown in Fig. 2.2.

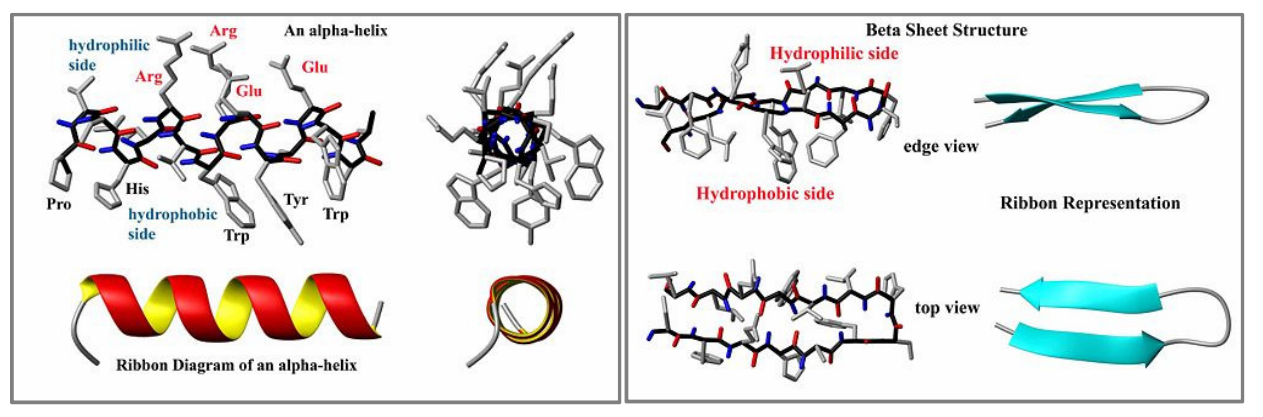

Fig. 2.2. The secondary structures of proteins, $\alpha$-helix and $\beta$-sheet (http://en.citizendium.org/wiki/protein_structure).

The tertiary structure is an overall folding of the protein into a more compact structure, mostly occurring via non-covalent forces and sometimes via disulfide bonds. The quaternary structure is a multi-chain association between several proteins. These 4 levels of structural organization result in the functionality and increased stability of the protein.

The biological function of most biomolecules depends on their ability to interact with other molecules. Therefore, it is of great interest to identify the epitopes - specific recognition sites, and binding sites, defining its core activity. Peptides, being fragments of a protein, are shorter and only have a linear structural organization. Furthermore they can be synthesized in-situ like DNA oligonucleotides. As an example, high density peptide microarrays can be fabricated by direct synthesis of peptides on a surface using photolithography or light-directed synthesis ${ }^{5}$, this is further explained in section 2.3.3. 


\subsubsection{Antibodies \& autoimmune diseases}

Antibodies and immune responses ${ }^{67}$ are widely studied and used in biological research ${ }^{8}$. Antibodies or immunoglobulins are high molecular weight proteins of approximately 150 $k D a$, consisting of four subunits including two heavy chains and two light chains (as shown in Fig. 2.3). Antibodies are intended to keep an organism free from foreign entities, and to remove abnormalities from within an organism. They are normally produced by specialized B-lymphocytes after stimulation by an antigen, a substance foreign to the human body, such as an immunogen or a hapten. Within an immune response antibodies act specifically against the antigen to remove it from the organism.

A normal immune response would lead to the production of polyclonal antibodies which are directed against multiple epitopes of the antigen as they are processed by antigen presenting cells and B-lymphocytes. The name B-lymphocyte originates from Bursa of Fabricius, an organ in birds where the B-lymphocytes mature. B-lymphocytes are produced in the bone marrow. Monoclonal antibodies on the other hand, are derived from fusing spleen cells with a myeloma cell line. These cell lines, produce identical antibodies which are used in therapeutics and research because of their uniform affinity and specificity.

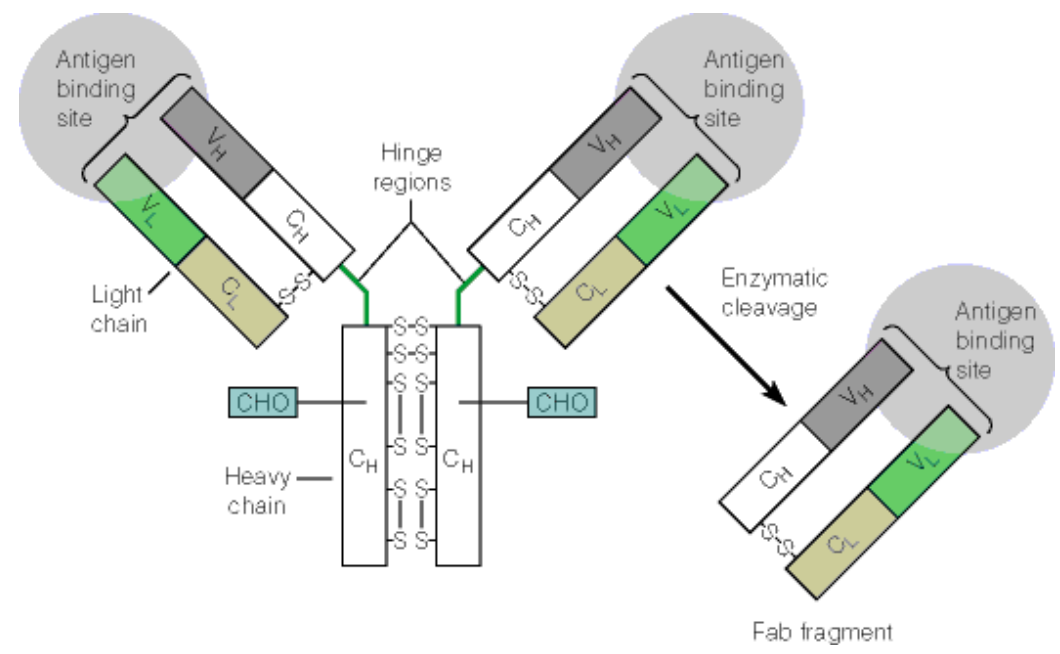

Fig. 2.3. Schematic representation of an antibody consisting of 2 heavy $\left(C_{H}+V_{H}\right)$ and 2 light chains $\left(C_{L}+V_{L}\right)$ having a constant domain (C) and a highly variable domain (V) binding the antigen. The carbohydrate binding domain $(\mathrm{CHO})$ plays a role in the protein targeting and lifetime of the antibody in the serum. The disulfide bonds, represented by -S-S- , provides the antibody extra stability ${ }^{3}$. 
If the delicate balance of antigen recognition and antibody production becomes disordered, allergies or autoimmune diseases, may result ${ }^{7}$. A hallmark of autoimmune diseases is the production of high-affinity autoantibodies, coordinated by B- and Tlymphocytes expressing a diverse repertoire of antigen receptors ${ }^{9}$. These circulating autoantibodies bind to self-proteins and thereby attack the species at an organ or systemic level. Since there is no fundamental difference between the structure of selfantigens and that of foreign antigens, lymphocytes evolved to respond only in certain microenvironments, generally in the presence of inflammatory cytokines. Many triggers have been appointed to increase the susceptibility to autoimmune diseases, e.g. genetic factors, environmental triggers, microbes, estrogens and drugs ${ }^{10}$. Furthermore, for many diseases these autoantibodies can be found in serum samples many years before disease onset. Identification of the presence of these autoantibodies might allow immunological treatment whereby the disease is prevented or life-threatening conditions can be avoided ${ }^{11}$. Therefore, reproducible and reliable serological and clinical methods assessing the risk of a specific disease and identifying active disease and remission is important. The study of rheumatoid arthritis specific autoantibody presence in serum samples, is discussed in more detail in chapter 5 .

\subsubsection{Proteomics}

The word proteomics was coined in the early 1990s by the PhD candidate Marc Wilkins as an alternative for the phrase "the protein complement of the genome" and added to the Merriam-Webster dictionary in 1997 as follows:

Pro•te•o•mics (prō-tē-'o-miks): a branch of biotechnology concerned with applying the techniques of molecular biology, biochemistry, and genetics to analyzing the structure, function, and interactions of the proteins produced by the genes of a particular cell, tissue, or organism, with organizing the information in databases, and with applications of the data $^{12}$.

The large-scale analysis of proteins will contribute greatly to our understanding of gene function in the post-genomic era as the genetic code alone will not provide any information regarding their regulation. The field of proteomics can be roughly divided into two broad areas, discovery oriented (the search for new proteins) and systems oriented (the understanding of the relation between proteins) ${ }^{13}$. The conventional proteomics 
approach is discovery oriented, which dates back to the late 1970s with the development of the 2-D gel electrophoresis ${ }^{14}$. It is the separation, followed by the quantification and identification of as many proteins as possible within a biological sample, typically performed on a 2-D gel electrophoresis combined with mass spectrometry (MS) ${ }^{15}$. Analyzing proteins in complex biological samples is not without bottlenecks as the dynamic range of protein abundance can be as high as $10^{10}$. Moreover, protein concentrations vary tremendously, the differential concentration of abundant and rare body fluid proteins can exceed $12 \operatorname{logs}^{16}$. Since the best 2D-gels cannot resolve more than $10^{4}$ proteins, it is obvious that only the most abundant ones can be visualized if a crude protein mixture is used.

In 2-D gel electrophoresis, the proteins are electrophoretically separated and quantified in two steps, to obtain an increased resolution. The first dimension separation is based on the isoelectric point (PI) of the protein, by isoelectric focusing (IEF). IEF is based on the fact that a molecule's charge changes upon the $\mathrm{pH}$ of its surrounding, being positively charged if the $\mathrm{pH}$ is below the $\mathrm{PI}$ and vice versa. The second dimension separation is based on the proteins mass, on a sodium dodecyl sulfate polyacrylamide gel electrophoresis (SDSPAGE). The SDS, denatures the disulfide bonds of the proteins, and results in reattaining its primary linear structure. In this linear structure the separation by PAGE is purely based on the difference in mass. After isolating the separated proteins from the gel, they are digested into peptides by a sequence specific enzyme and analyzed by MS ${ }^{17}$. MS is an analytical technique to identify the chemical composition of a compound based on the mass-to-charge ratio. This unfocused screening of known and unknown proteins does not allow high-throughput screening (HTS), because the relative location of the proteins varies between experiments. Even though 1,000 peptide spectra can be obtained per hour, they need to be scanned against comprehensive protein sequence databases for identification in order to find the few interesting or new proteins.

The systems-oriented proteomics approach consists of studying known proteins that are related to each other by function or sequence and their interactions with each other. As mentioned before, genome sequencing projects have contributed greatly to the functional analysis of proteins. There are many techniques available for the analysis of specific interactions. Some examples of protein-detecting assays contributing to quantify the proteins within a biological sample are, antigen precipitation, radioimmunoassay (RIA) ${ }^{18}$, enzyme-linked immunosorbant assay (ELISA) ${ }^{19}$ and microarrays. RIA is a competitive 
assay, in which a known amount of radio-labeled antigen is mixed with the sample. In an ELISA assay, an antigen is immobilized in a microtiter plate and forms a complex with a specific antibody, a fluorogenic or chromogenic secondary antibody binds the detecting antibody. The ELISA assay is a commonly used high-throughput analysis method, however the separate wells are all microenvironments, where a microarray only has one sample well making it a true parallel high-throughput analysis method. Microarrays will be further discussed in section 2.3 and chapter 3 .

\subsection{Label-free biosensing}

A biosensor is a device that monitors and transmits information about a biological process, e.g. an analyte that reacts with a target substance and a signal-generating electrochemical component that detects the resulting product.

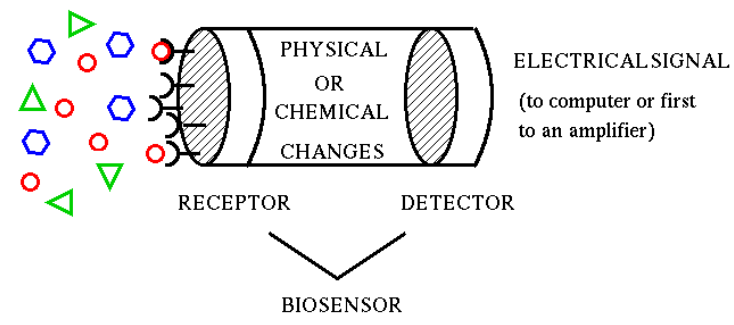

Fig. 2.4. Schematic representation of the working principle of a biosensor (by Urban Jenelten).

There are multiple types of biosensors, e.g. electrochemical, acoustic, mechanical, and optical $\begin{array}{lll}20 & 22 & 22\end{array}$. Although a large variety of research is carried out on biosensors, we consider only surface plasmon resonance, an optical biosensor.

\subsubsection{Label vs. label-free}

There are generally two types of detection methods: label-based detection and label-free detection ${ }^{23}$. Label-based detection use a variety of labels including radioactive, magnetic 24 , chromogenic, fluorescence-based and quantum dots, of which fluorescent labels are currently most commonly used. Advantages of fluorescence-based detection are its high 
sensitivity, with detection limits down to a single molecule ${ }^{25}$, availability of a wide range of commercially available labels and well-established detection techniques based on optical microscopy. By combining different labels, multicolored and high quality microscope images provide valuable information about the life processes. Photobleaching can be a problem in fluorescence-based detection techniques, however, it can also be exploited to study the dynamics inside living cells e.g. FRAP (fluorescence recovery after photobleaching) and FLIP (fluorescence loss in photobleaching) ${ }^{26}$. New and more stable dyes are being developed, as well as quantum dot nanoparticles, which can eliminate photobleaching and open doors for new methods, such as optical bar coding ${ }^{27}$. However, labels may interfere with the biological function of the biomolecule and quantitative analysis can be difficult as the number of fluorophores on each molecule is difficult to control $^{23}$.

In the case of label-free biosensing the analyte does not require any specific characteristics or labels, and there is no need for multistep detection protocols like sandwich assays ${ }^{28}$, even though sandwich assays can provide additional specificity. Moreover, label-free biosensing allows real-time monitoring of the binding occurrence, providing additional information about the interaction, such as affinity, specificity, quantitative and kinetic or thermodynamic data ${ }^{29}$. Miniaturization is also possible, which may lead to point-of-care instrumentation ${ }^{30}$. The selectivity and sensitivity are currently actively debated among the biology community, and they are only slowly accepting these newly developed methods.

Label-free optical detection techniques can be divided into a number of categories, for example refractive index changes, optical absorption and Raman detection. Refractive index based label-free detection suitable for biosensing is based on e.g. surface plasmon resonance, interferometer, waveguide, ring resonator, optical fiber or photonic crystals ${ }^{23}$. Surface plasmon resonance is currently the most mature label-free technique with the invention of SPR imaging ${ }^{31}$ simultaneous biomolecular interactions can be measured, which is important for proteomic applications as mentioned previously. Therefore we will only discuss the SPR imaging technique used for label-free biosensing in further detail. 


\subsubsection{Surface plasmon resonance}

Surface plasmon resonance (SPR) biosensors enable today's research to explore the kinetics of biomolecular interactions ${ }^{32}$. The physical phenomenon of surface plasmon resonance was first observed in $1902^{33}$, however a complete understanding only arose in $19688^{34}$. The use of SPR for biosensing, first mentioned in the literature in the $1980 \mathrm{~s}^{36} 37$, allows label-free and real-time detection of various biomolecular interactions ${ }^{38} 3940$.

Many different SPR platforms and technology combinations have been described ${ }^{41}$ : e.g., SPR imaging ${ }^{31424344}$ SPR microscopy ${ }^{45} 46$, surface plasmon field-enhanced fluorescence spectroscopy (SPFS) ${ }^{47} 48$, scanning electrochemical microscopy-SPR (SECMSPR) ${ }^{49}$, gratingcoupled SPR (GC-SPR) ${ }^{505152}$ and waveguide-coupled SPR (WCSPR) ${ }^{53}$.

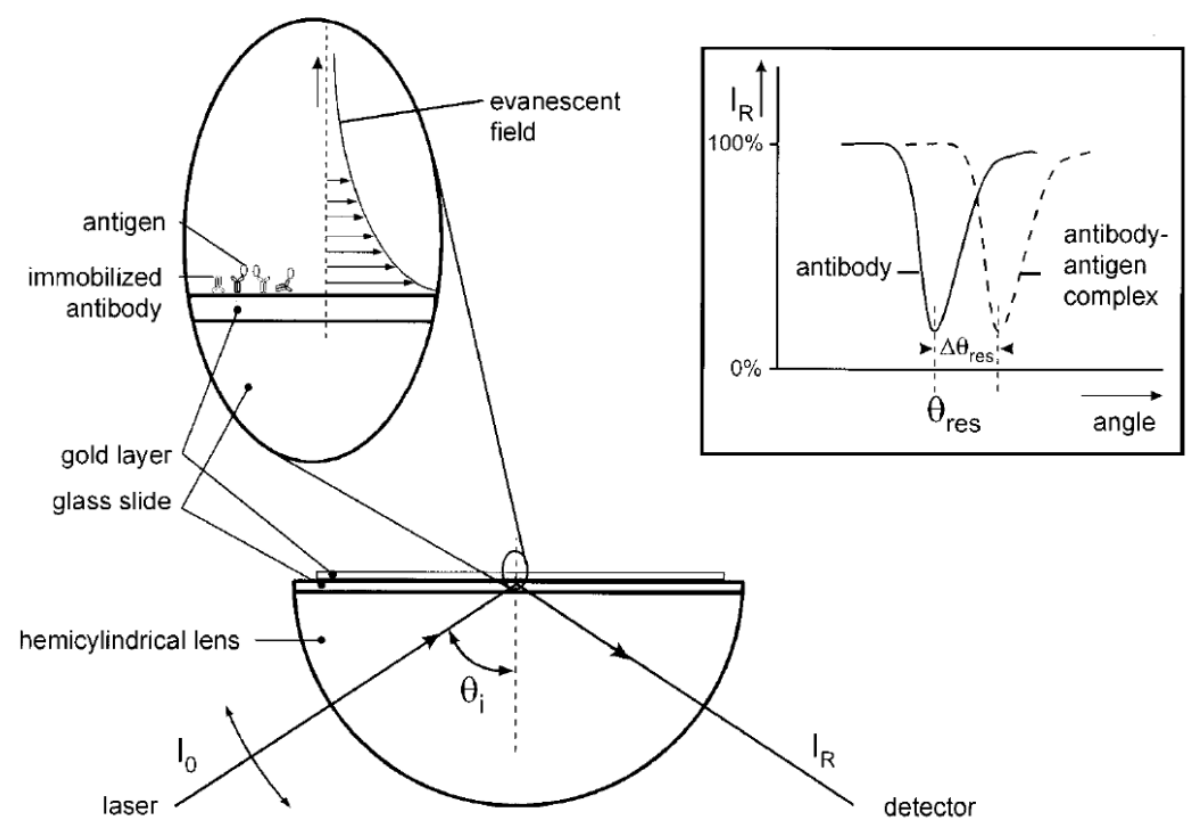

Fig. 2.5. Schematic representation of the SPR principle ${ }^{54}$ in the Kretchmann configuration. $I_{0}$ : laser excited signal, $I_{R}$ : reflected signal, $\theta_{i}$ : angle at incidence, $\theta_{\text {res }}$ : resonance angle used, $\Delta \theta_{\text {res }}$ : change in resonance angle.

The SPR principle is based on the excitation of surface plasmons at the surface of a thin layer $(\approx 50 \mathrm{~nm}$ ) of a metal such as gold, using ( $p-)$ polarized light with a coupling prism. At a certain angle of incidence, the free electron oscillation at gold and sample interface 
reaches a maximum and an evanescent field can be enhanced by a factor of around $30^{55}$ ${ }^{37}$. The actual angle of the so-called SPR-dip depends on the wavelength of the excitation source and the refractive indexes of all the layers e.g. prism, gold and sample layer. The penetration depth of the evanescent field into the sample is a function of the wavelength of the incident light, typically in the order of half a wavelength. The evanescent field does also penetrate into the thin gold layer but to a much smaller extent.

Analyte binding onto the sensor surface will induce a change of the refractive index at the gold-liquid interface, resulting in an angle shift of the SPR-dip. SPR measurements carried out in a fixed angle mode often translate the angle shift of the SPR-dip into a reflectivity signal calculated from the linear part of the SPR-curve ${ }^{56}$. These reflectivity signals show linearity up to $5 \%$ change in reflectivity ${ }^{57}$, which is equal to a $50 \mathrm{~m}^{\circ}$ angle shift of the SPRdip. The actual shift in SPR-dip or the derived parameter (change in reflectivity) followed as a function of time is plotted in a so called sensorgram. The sensorgram can be either a translation of the reflectivity change or a change in angle, as shown in Fig. 2.6. Due to the real-time recording of the change at the sensor surface, the sensorgram contains kinetic information, providing additional information about the concentration, affinity, and thermodynamics of the interaction and will be further described in chapter 6 .

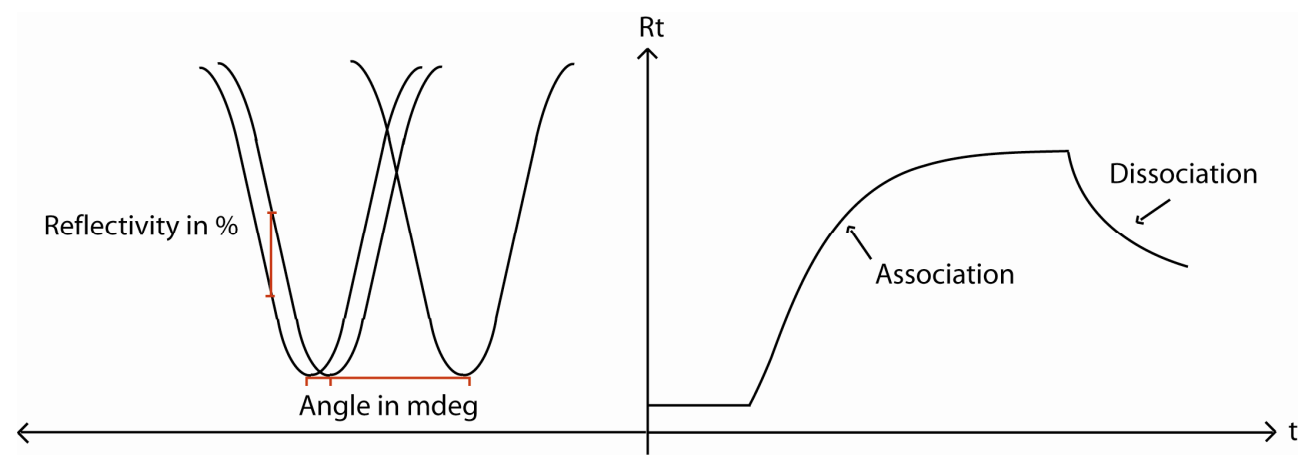

Fig. 2.6. Shift of the SPR dip in reflectivity or angle, and the translation into a sensorgram. Small changes in the SPR dip can be measured more accurately by the change in reflectivity, however, large changes cannot be measured due to the loss of linearity. The change in angle is less sensitive for very small changes, however, remains linear and can therefore measure very large changes in the SPR dip. This is explained in more detail in section 4.3.1 and Fig 4.3. 
The most-common SPR light coupling configuration is the so-called Kretschmann configuration ${ }^{35}$, in which a high refractive index prism directs incident light to thin gold layer and surface plasmons are generated at the opposite gold surface at the sample interface and reflects at the gold surface without traveling through the liquid. Another SPR coupling configuration is grating-coupled SPR configuration where the incident light passes through the sample before reflecting at the grating 505152 , and therefore sample solution and flow-cell need to be optically transparent ${ }^{58}$.

In the case of SPR imaging, a collimated excitation beam with large uniform spot areas is used to image the sensor surface by a charge-coupled device (CCD) camera. Multiple regions of interest (ROIs) can be positioned to measure and calculate the change in reflectivity or angle at hundreds to thousands of spots simultaneously. Scanning-angle surface plasmon resonance imaging and its applications when combined with microarray technology, is in further detail described in chapters 4,5 and 6 of this thesis.

\subsection{Microarrays}

As mentioned in section 2.1.3, a focused or systems-oriented proteomics approach, studying known proteins that are related to each other by function or sequence, can be performed using microarrays ${ }^{13}$. Other important application areas of protein microarrays are diagnostics ${ }^{9}$, proteomics and therapeutics ${ }^{59}$.

A microarray consists of numerous biomolecules immobilized to a solid support in a regular pattern. Fluorescently labeled targets introduced to the surface immobilized molecules where binding or hybridization occurs between matching ligand-analyte pairs only. The unbound molecules are washed away and an optical scanner is used to measure the optical signal from the fluorescent labels. This is a common high-throughput technique used in molecular biology. In Fig. 2.7 an example of a typical microarray assay where labeled complementary DNA (cDNA) probes are hybridized to a microarray containing single-stranded DNA (ssDNA) is shown. Microarrays originated in the 1970s, in the form of the Southern blot to study DNA-DNA interactions ${ }^{60}$. Separated DNA was transferred to a nitrocellulose membrane acting as a solid support followed by an interaction with radio-labeled complementary DNA. Soon after, the technique was applied for the study of other binding interactions e.g. northern blot for DNA-RNA, western blot 
for protein-protein and northwestern blot for DNA-protein. The attempt to make arrays of DNA, RNA, peptides or proteins and to gain as much information as possible from a single test or experiment has become a huge challenge within the scientific world. In the past years, protein microarray technology has shown great potential in basic research, diagnostics and drug discovery as the genome cannot provide enough information to fully understand the complex cellular network. Not only the spotting technique, also the immobilization chemistry used is very important for the manufacturing of high quality microarrays.

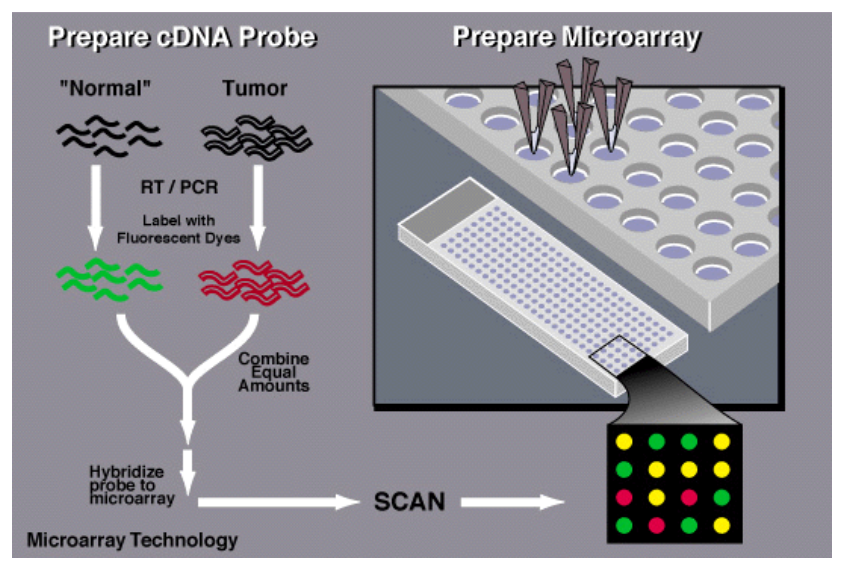

Fig. 2.7. Microarray technology: an example of how to prepare a cDNA microarray (http://www.genome.gov/Pages/Hyperion/DIR/VIP/Glossary/Illustration/microarray_technology.cfm).

\subsubsection{Immobilization chemistry}

Immobilization chemistry or surface coupling methods greatly influence how the ligands link to the surface in terms of density, orientation and bond strength. One way to couple ligands to the surface is by van der Waals forces, resulting in weak, short-range electrostatic attractive forces between uncharged molecules, which arise from the interaction of permanent or transient electric dipole moments. They are not often used to couple molecules to surfaces as a change in $\mathrm{pH}$ or salt concentration commonly results in their removal. Covalent or chemical coupling provides a more stable and often a orientation controlled coupling of the ligand to the surface. Covalent coupling taking 
advantage of an un-reacted amino group (N-terminus) or carboxyl group (C-terminus) of a protein is called amino coupling, shown in Fig. 2.8. 1-ethyl-3- (3-dimethylaminopropyl) carbodiimide hydrochloride (EDC) and N-hydroxysuccinimide (NHS) is often used to couple the $\mathrm{N}$-terminus of a peptide or protein to a carboxylated surface.

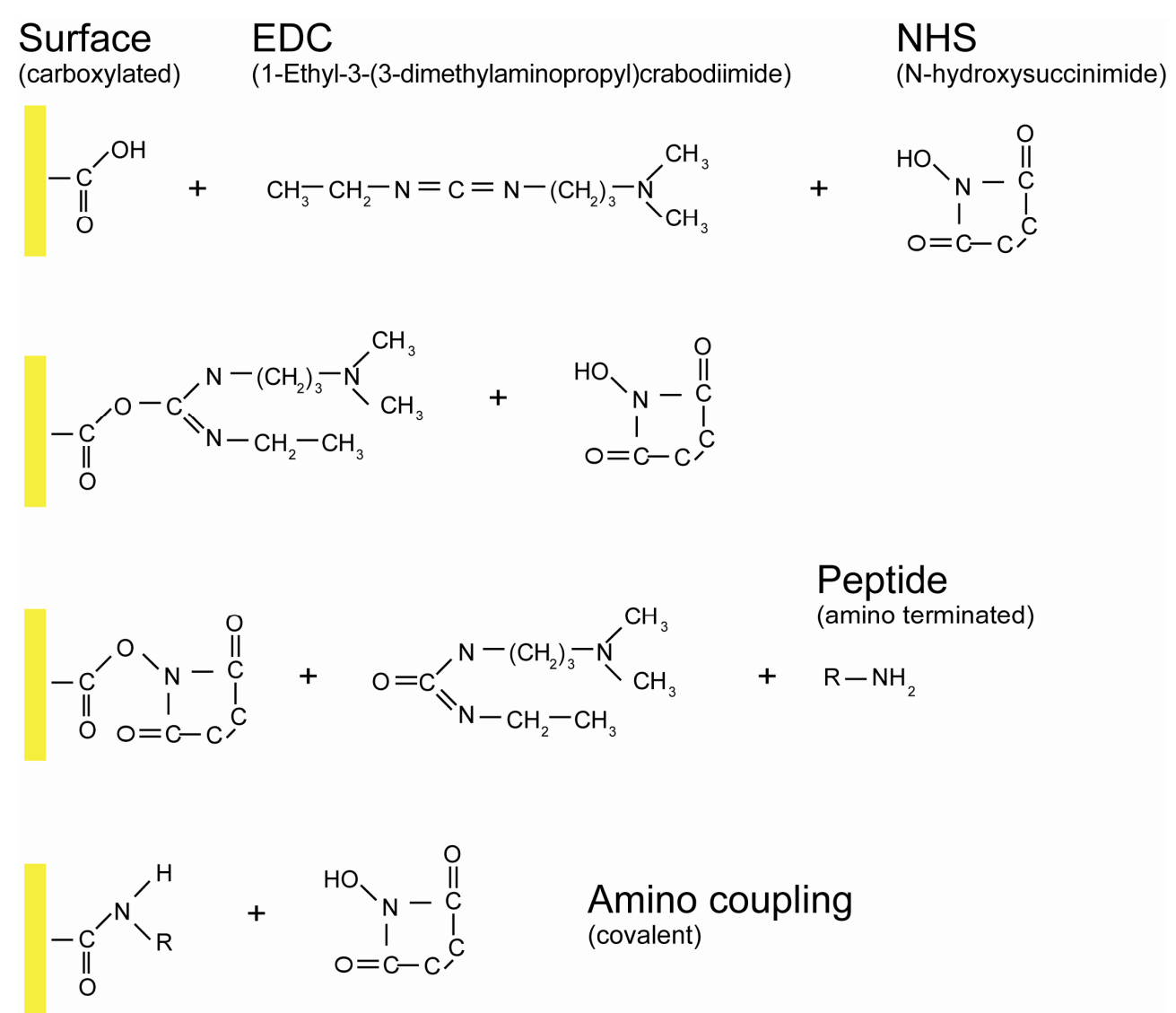

Fig. 2.8. reaction scheme of the amino coupling with EDC / NHS.

Other common coupling chemistries are e.g. thiol coupling and aldehyde coupling. High affinity capture coupling is mostly not resistant to regeneration conditions, and the ligand has to be immobilized prior to every analysis cycle. Affinity is defined by the strength of binding between a ligand and its analyte, the higher the affinity, the lower the value found for the dissociation constant $\left(K_{D}\right)$ as this indicates the decreased likelihood of separation 
of the ligand-analyte complex. In the case of biotin-streptavidin, of which the affinity is $K_{D}$ $\approx 10^{-15} \mathrm{M}$, the bond is considered to be irreversible ${ }^{61}$. Therefore biotin-streptavidin is often used to immobilize DNA molecules to the surface. The properties of the ligand have to be taken into account when deciding which approach to take, especially in the case of fragile proteins.

\subsubsection{Immobilization density}

A well controlled ligand density on a biosensor surface is of high importance for obtaining reproducible results. The ligands are mostly diluted with a buffer in which they are immobilized in an attempt to create different densities on the surface. However, by doing so the immobilization rate, total amount of available molecules in solution combined with the available positions on the surface and the time given are all responsible for the obtained result. A better control of the ligand density can be obtained when a background molecule is used for dilution with a similar binding rate to the surface. In chapter 6 we will discuss this in more detail combined with a possible application, kinetic analysis by means of ligand densities. We also compare the standard analyte overlay plots used to calculate the kinetic constants, with the new approach using various ligand concentrations. This new approach could provide a higher-throughput kinetic analysis at microarrays without the need for regeneration and thereby reducing the analysis time.

\subsubsection{Spotting techniques}

As mentioned before, a microarray consists of numerous molecules attached to a solid support in a regular pattern. This pattern consists of many, so-called spots, consisting of only one type of molecule. The delivery of the droplet containing these molecules to the surface of the solid support, is called spotting or printing. The various spotting techniques can be roughly divided into two groups, the contact and the non-contact spotters.

Contact spotters or pin tools take advantage of capillary forces when releasing droplets by touching the surface, therefore the fluid properties are a very important parameter contributing to the actual spot size besides the surface itself. There are various types of pins available, of which the solid pins and split pins are the most important. The split pin is mostly used as they are hollow and can take up to $1.25 \mu \mathrm{l}^{62}$ of sample to continuously produce spots on the surface, whereas the solid pins have to be reloaded with new 
material after every deposition. If controlled very precisely, the split pins can be considered to be non-contact spotters.

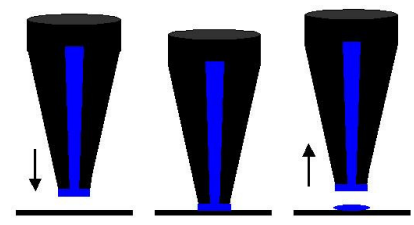

Fig. 2.9. The working procedure of a new type of split pin, the Stealth pin from TeleChem ${ }^{62}$

Microarrays can also be fabricated using atomic force microscope tips (AFM tips), by dip pen nanolithography (DPN) ${ }^{63}$. Commercial printing examples are the DPN (Nanolnc, Inc., USA), and the Nano eNabler (Bioforce Nanosciences, Inc., USA), both produce spot sizes still in the micometer range, $1-60 \mu \mathrm{m}$. There are also cases of nanografting ${ }^{64}$ reaching a resolution within the nano-range $\leq 100 \mathrm{~nm}$.

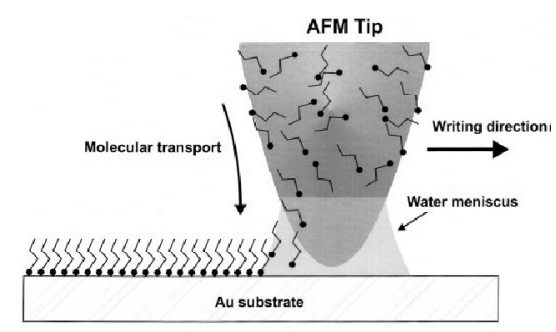

Fig. 2.10. The principle of the dip-pen is deposition of molecules on a plain surface. In the case of nanografting, molecules of the surface are being replaced by the AFM tip containing a different type of molecules ${ }^{63}$.

Another contact spotting technique is based on the transfer of molecules from one surface to another, called $\mu$ contact printing ${ }^{65}$. Conventional examples of microcontact printing with elastomeric stamps, are not really applicable for producing microarrays that contain a high variety of ligands, and are primarily interesting for surface modification purposes ${ }^{66}$. There are also various microfluidic spotting techniques available based on polydimethylsiloxane (PDMS, Sylgard ${ }^{\circledR} 184$ Silicone Elastomer) as they are designed to be disposable. The use of microfluidic PDMS channels to immobilize ligands on a surface has a lot of potential ${ }^{6768}$ and is explained in further detail in chapter 3. 
The non-contact spotters deposit molecules on the surface without touching the surface. In the case of inkjet printing, a droplet is ejected after compression of the air in the actuation chamber using a piezoactuator. Here the velocity is very important for the actual spot size, in addition to the fluid and surface properties. The commercially available Topspot ${ }^{69}$ is described in more detail in chapter 3. Inkjet printing can also be used for insitu synthesis of oligonucleotides by depositing molecules one base at a time at precise locations ${ }^{70}$. A more general photolithographic adsorption technique uses a photo mask to synthesize the molecules in-situ ${ }^{5}$, of which a schematic representation can be seen in the Fig. 2.11.

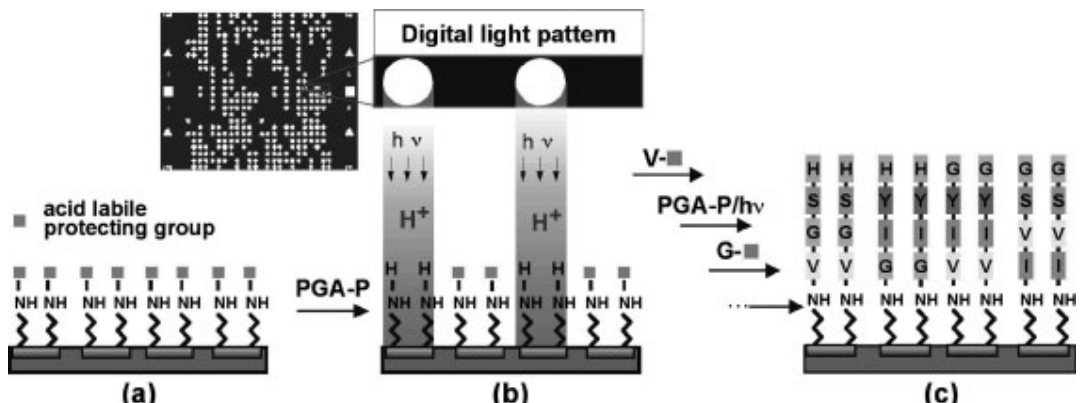

Fig. 2.11. A schematic representation of microarray fabrication by in-situ synthesis by photolithography ${ }^{5}$ (can be combined with inkjet spotting)

The shape and homogeneous dispersion of the molecules within the spots is highly dependent on the spotting procedure, used. Undesired "donuts" are easily formed during the drying process, with the immobilized molecules located in a ring around the circumference of the spot. The drying process is, therefore, an important and critical step in manufacturing microarrays, especially in the case of protein immobilization it is important to preserve their active state.

The detection techniques used for analyzing microarrays are mainly based on labeled detection techniques and fluorescence imaging with high-throughput optical scanners, or label-free techniques, SPR imaging or cantilevers ${ }^{71}$. The combination of SPR imaging and microarrays will be discussed in more detail in chapter 4, 5 and 6 . Various ligand immobilization techniques are discussed in chapter 3. 


\subsection{Data analysis}

Although often overlooked, data analysis is one of the most important aspects of the (bio)assay. Extracting and interpreting the measured data is rarely straight forward. Especially when large data sets have to be analyzed, data mining, becomes very important to sort out the relevant information. High-throughput assays generate large amounts of data that need to be analyzed in short periods of time often relying on computing power and sophisticated algorithms. The importance of bioinformatics is becoming clear, with the choice of available software increasing.

\subsubsection{Interpreting microarray data}

The initial information obtained from a microarray experiment is a "yes" or "no" answer to molecular binding. Dependent on the experimental design and analysis technique used, more information can be hidden within the results of an assay, e.g. analyte concentration, specificity and affinity. By applying bioinformatics a new field of science specialized in handling and analyzing enormous databases generated by arraying techniques, correlations between various samples can be found, thereby increasing the information of an experiment ${ }^{72}$. Fig. 2.12 shows an example of an heat map analysis, in which the results of 2 microarray chips are compared. By using fluorescence based detection of the interaction at a microarray, a static end result of the presence and binding of the molecules can be obtained. By making an overlay of two experimental data sets, labeled with different colors e.g. Cy3 (green) and Cy5 (red), information of up- and downregulation can be obtained ${ }^{74}$. 


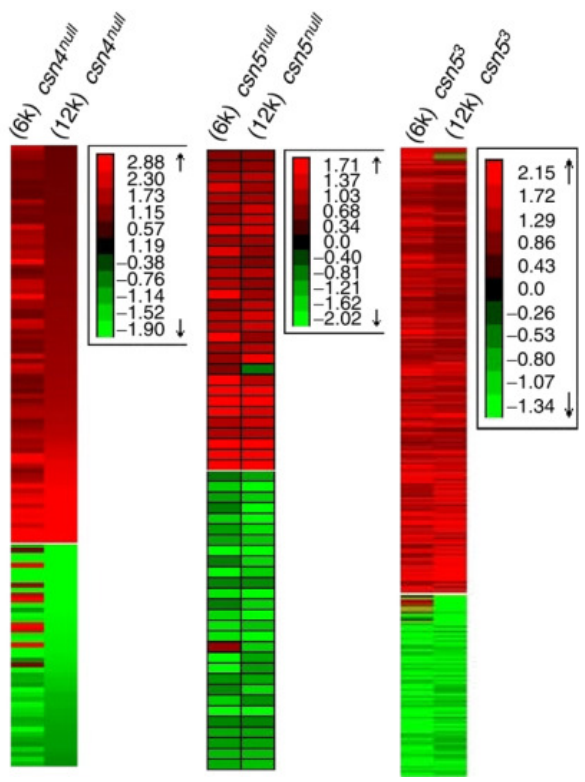

Fig. 2.12. Heat map data analysis of Drosophila melanogaster microarray ${ }^{73}$. The results of a $6 \mathrm{k}$ and a $12 \mathrm{k}$ microarray chip are being compared with each other. The difference in gene expression is depicted in green (down-regulation) and red (up-regulation).

\subsubsection{Interpreting SPR data}

As mentioned before, SPR imaging data provides real-time information of biomolecular interactions in a label-free manner. The measured sensorgram can be divided in various parts, e.g. baseline, association, dissociation and regeneration. All of them contain valuable information concerning the biomolecular interaction of the analyte and the ligand, as shown in Fig. 2.13. 


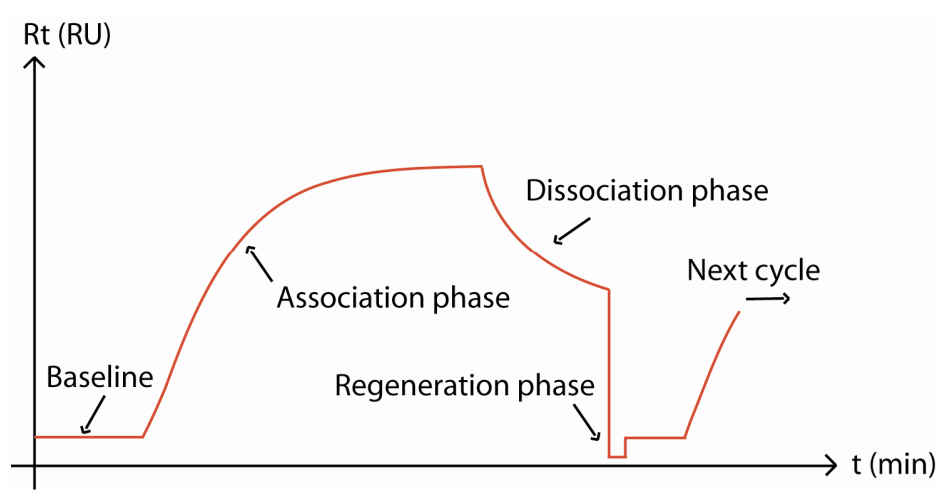

Fig. 2.13. Schematic representation of an SPR sensorgram.

The response Rt, is an indication of the amount of molecules bound to the surface compared to the baseline situation. The time $t$, required to obtain a certain response depends on the concentration of both analyte and ligand. Furthermore, it indicates how fast the interaction proceeds and provides valuable thermodynamic information if interactions at different temperatures are performed ${ }^{75} 76$. The profile of the association phase contributes to the association rate $k_{a}$ but mainly to the concentration of the analyte. The dissociation phase is merely an indicator of the dissociation rate $k_{d}$, the strength of the bond of the interacting molecules. The combined behavior of association and dissociation results in the equilibrium constants $\mathrm{K}_{\mathrm{A}}$ and $\mathrm{K}_{\mathrm{D}}$

$$
\begin{gathered}
K_{A}=\frac{k_{a}}{k_{d}} \\
K_{D}=\frac{k_{d}}{k_{a}}
\end{gathered}
$$

Currently, the most common approach to modeling measured SPR data is to begin with a 1:1 interaction model,

$$
A+B \underset{k_{d}}{\stackrel{k_{a}}{\rightarrow}} A B
$$


where $A$ is the analyte and $B$ is the immobilized ligand, and $A B$ the complex formed at the sensor surface. Of course, this depends on the ligand-analyte behavior and mass-transport behavior which can have a profound effect on the interpretation of measured data. The 1:1 interaction model is based on the Langmuir isotherm ${ }^{77}$.

$$
\theta=\frac{\alpha A}{1+\alpha A}
$$

$\theta$ is the percentage of surface coverage at equilibrium, $\alpha$ the Langmuir adsorption constant $\left(\mathrm{K}_{\mathrm{A}}\right)$ and $\mathrm{A}$ the pressure or analyte concentration.

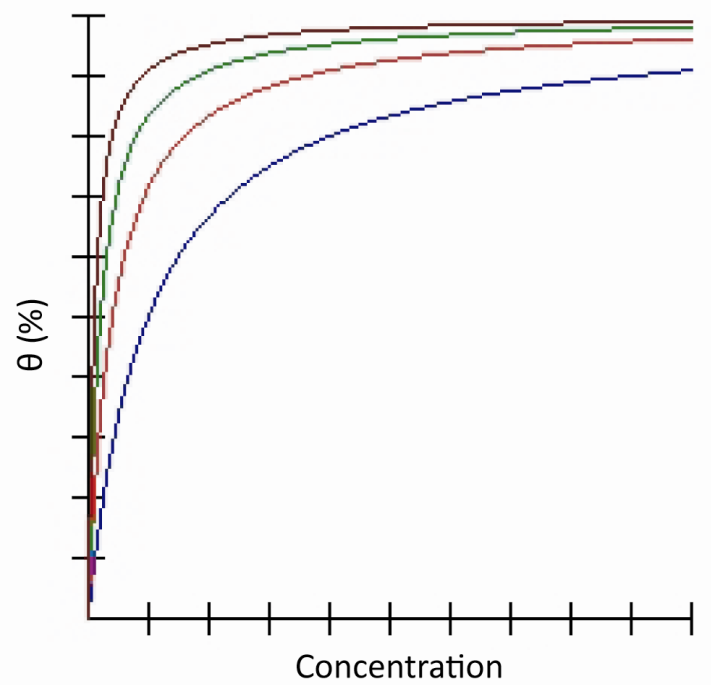

Fig. 2.14. Langmuir isotherm curve.

If the data can be fit to the $1: 1$ interaction model, then a simple bimolecular interaction is assumed to take place, requiring only one bond to form $A B$ complex. However, conformational changes, mass transport limitations due to surface geometry (steric hindrance) and surface heterogeneity are well known factors that result in a poor fitting to the model. For more detailed information about kinetic analysis in combination with SPR the reader is referred to chapter 6 , where the kinetic analysis of microarray based data obtained with SPR imaging is being discussed ${ }^{78} 79$. 


\subsection{Conclusions}

Combining (old and new) techniques can provide valuable tools to investigate protein interactions. SPR imaging is a powerful tool to study biomolecular interactions in a labelfree and real-time manner. Here we explore the usability of peptide microarrays for the detection of autoantibodies present in serum samples, to provide a high-throughput labelfree alternative to conventional assays, such as ELISA.

Both, spotting techniques as well as data analysis are important for obtaining high quality data and bioinformatics is getting a more prominent role in the analysis of highthroughput screening.

\subsection{References}

1 Hood, L.; Heath, J.R.; Phelps, M.E.; Lin, B., Systems biology and new technologies enable predictive and preventive medicine. Science 306, 640643 (2004)

2 Davies, J.S.; Chan, W.C., Amino Acids, Peptides and Proteins. (Royal Society of Chemistry, 2006).

3 Mathews, C.K.; Holde, K.E. van, Biochemistry, second ed. (Menlo Park, CA : Benjamin Cummings, 1996).

4 Meyers, R.A., Proteins: From Analytics to Structural Genomics. (Wiley-VCH, 2006).

5 Gao, X.; Zhou, X.; Gulari, E., Light directed massively parallel on-chip synthesis of peptide arrays with t-Boc chemistry. Proteomics 3, 2135-2141 (2003).

6 Roitt, I.; Brostoff, J.; Male, D., Immunologie, 4th ed. (Bohn Stafleu Van Loghum, Houten/Diegem, 1996).

7 Shoenfeld, Y.; Gershwin, M.E.; Meroni, P.L., Autoantibodies, second ed. (Elsevier Inc., 2007).

8 Goding, J.W., Monoclonal Antibodies, Principles and Practice, third ed. (Elsevier Ltd, 1996). 
9 Robinson, W.H.; DiGennaro, C.; Hueber, W.; Haab, B.B.; Kamachi, M.; Dean, E.J.; Fournel, S.; Fong, D.; Genovese, M.C.; Neuman de Vegvar, H.E.; Skriner, K.; Hirschberg, D.L; Morris, R.I.; Muller, S.; Pruijn, G.J.; Venrooij, W.J.van; Smolen, J.S.; Brown, P.O.; Steinman, L.; Utz, P.J., Autoantigen microarrays for multiplex characterization of autoantibody responses. Nature Medicine 8 (3), 295-301 (2002).

10 Davidson, A.; Diamond, B., Autoimmune diseases. The New England Journal of Medicine 345 (5), 340-350 (2001).

11 Scofield, R.H., Autoantibodies as predictors of disease. The Lancet 363, 15441546 (2004).

12 Merriam-Webster, edited by Incorporated Merriam-Webster (Springfield, MA, USA, 2005).

13 MacBeath, G., Protein microarrays and proteomics. Nature Genetics Supplement 32, 526-532 (2002).

14 O'Farrell, P.H., High resolution two-dimensional electrophoresis of proteins. Journal of Biological Chemistry 250 (10), 4007-4021 (1975).

15 Pandey, A.; Mann, M., Proteomics to study genes and genomes. Nature 405 (15 june 2000), 837-846 (2000).

16 Hochstrasser, D.F.; Sanchez, J.-C.; Appel, R.D., Proteomics and its trends facing nature's complexity. Proteomics 2, 807-812 (2002).

17 Aebersold, R.; Mann, M., Mass spectrometry-based proteomics. Nature 422, 198-207 (2003).

18 Yalow, R.S.; Berson, S.A., Immunoassay of endogenous plasma insulin in man. The Journal of Clinical Investigation 39 (7), 1157-1175 (1960).

19 Engvall, E.; Perlmann, P., Enzyme-linked immunosorbent assay (ELISA) quantitative assay of immunoglobulin G. Immunochemistry 8 (9), 871-874 (1971).

20 Knopf, G.K.; Bassi, A. S., Smart Biosensor Technology. (CRC Press, 2007).

21 Zhang, X.; Huangxian Ju, H.; Wang, J., Electrochemical Sensors, Biosensors and their Biomedical Applications. (Elsevier Inc, 2008).

22 Gauglitz, G., Direct optical sensors: principles and selected applications. Analytical and Bioanalytical Chemistry 381 (1), 141-155 (2005). 
Fan, X.; White, I.M.; Shopova, S.I.; Zhu, H.; Suter, J.D.; Sun, Y., Sensitive optical biosensors for unlabeled targets: a review. Analytical Chimica Acta 620, 8-26 (2008).

24 Aytur, T.; Foley, J.; Anwar, M.; Boser, B.; Harris, E.; Beatty, P.R., A novel magnetic bead bioassay platform using a microchip-based sensor for infectious disease diagnosis. Journal of Immunological Methods 314, 21-29 (2006).

25 Moerner, W.E., Single-molecule Chemistry and Biology Special Feature: New directions in single-molecule imaging and analysis. Proceedings of the National Academy of Sciences 104 (31), 12596-12602 (2007).

26 White, J.; Stelzer, E., Photobleaching GFP reveals protein dynamics inside live cells. Trends in Cell Biology 9 (2), 61-65 (1999).

27 Rosi, N.L.; Mirkin, C.A., Nanostructures in biodignostics. Chemical Review 105 (4), 1547-1562 (2005).

28 Boozer, C.; Kim, G.; Cong, S.; Guan, H.W.; Londergan, T., Looking towards label-free biomolecular interaction analysis in a high-throughput format: a review of new surface plasmon technologies. Current Opinion in Biotechnology 17, 400-405 (2006).

29 Rich, R.L.; Myszka, D.G., Why you should be using more SPR biosensor technology. Drug Discovery Today: Technologies 1 (3), 301-308 (2004).

30 Healy, D.A.; Hayes, C.J.; Leonard, P.; McKenna, L.; O'Kennedy, R., Biosensor developments: application to prostate-specific antigen detection. Trends in Biotechnology 25 (3), 125-131 (2007).

31 Rothenhauserler, B.; Knoll, W., Surface plasmon microscopy. Nature 332, 615-617 (1988).

32 Rich, R.L.; Myszka, D.G., Survey of the year 2005 commercial optical biosensor literature. Journal of Molecular Recognition 19, 478-534 (2006).

33 Wood, R.W. Philosophical Magazine 4, 396-402 (1902).

34 Otto, A. Zeitschift fur Physik A. 216, 398-410 (1968).

35 Kretschmann, E.; Reather, H., Radiative decay of non-radiative surface plasmons excited by light. Zeitschrift fur Naturforschung, Teil A. 23A, 21352136 (1968). 
36 Liedberg, B.; Nylander, C.; Lundstrom, I., Surface plasmon resonance for gas detecting and biosensing. Sensors and Actuators B chemical 4, 299-304 (1983).

37 Kooyman, R.P.H.; Kolkman, H.; Gent, J. van; Greve, J., Surface plasmon resonance immunosensors: sensitivity considerations. Analytica Chimica Acta 213, 35-45 (1988).

38 Lee, H.J.; Wark, A.W.; Corn, R.M., Creating advanced multifunctional biosensors with surface enzymatic transformation. Langmuir 22 (12), 52415250 (2006).

39 Kanda, V.; Kariuki, J.K.; Harrison, J.K; McDermott, M.T., Label-free reading of microarray-based immunoassays with surface plasmon resonance imaging. Analytical Chemistry 76, 7257-7262 (2004).

40 Homola, J., Present and future of surface plasmon resonance biosensors. Analytical Bioanalytical Chemistry 377, 528-539 (2003).

41 Wolfbeis, O.S.; Homola, J., Surface plasmon resonance based sensors. (Springer-Verlag, Berlin, 2006).

42 Wegner, G.J.; Wark, A.W.; Lee, H.J.; Codner, E.; Saeki, T.; Fang, S.; Corn, R.M., Real time SPR imaging measurements for the multiplexed determination of protein adsorption / desorption kinetics and surface enzymatic reactions on peptide microarrays. Analytical Chemistry 76 (5), 5677-5684 (2004).

43 Wolf, L.K.; Fullenkamp, D.E.; Georgiadis, R.M., Single nucleotidepolymorphism genotyping by nanoparticle-enhanced SPR imaging measurements of surface ligation reactions. Journal of the American Chemical Society 127, 17453-17459 (2005).

44 Huang, H.; Chen, Y., Label-free reading of microarray-based proteins with high throughput surface plasmon resonance imaging. Biosensors and Bioelectronics 22, 644-648 (2006).

45 Shumaker-Parry, J.S.; Zareie, M.H.; Aebersold, R.; Campbell, C.T., Microspotting streptavidin and double-stranded DNA arrays on gold for highthroughput studies of protein-DNA interactions by surface plasmon resonance microscopy. Analytical Chemistry 76, 918-929 (2004). 
Zang, T.; Morgan, H.; Curtis, A.S.G.; Riehle, M., Measuring particle-substrate distance with surface plasmon resosnance microscopy. Journal of Optics A: Pure and Applied Optics 3, 333-337 (2001).

47 Liebermann, T.; Knoll, W., Surface-plasmon field-enhanced fluorescence spectroscopy. Colloids and Surfaces A: Physicochemical and Engineering Aspects 171, 115-130 (2000).

Yu, F.; Yao, D.; Knoll, W., Surface plasmon field-enhanced fluorescnece spectroscopy studies of the interaction between an antibody and its surfacecoupled antigen. Analytical Chemistry 75, 2610-2617 (2003).

Xiang, J.; Guo, J.; Zhou, F., Scanning electrochemical microscopy combined with surface plasmon resonance: studies of localized film thickness variations and molecular conformation changes. Analytical Chemistry 78, 1418-1424 (2006).

50 Usui-Aoki, K.; Schimada, K.; Nagano, M.; Kawai, M.; Koga, H., A novel approach to protein expression profiling using antibody microarrays combined with surface plasmon resonance technology. Proteomics 5, 23962401 (2005).

51 Hultschig, C.; Kreutzberger, J.; Seitz, H.; Konthur, Z.; Bussow, K.; Lehrach, H., Recent advances of protein microarrays. Current Opinion in Chemical Biology 10, 4-10 (2006).

52 Singh, B.K.; Hillier, A.C., Surface plasmon resonance imaging of biomolecular interactions on a grating-based sensor array. Analytical Chemistry 78, 20092018 (2006).

53 Chien, F.-C.; Chen, S.-J., A sensitivity comparison of optical biosensors based on four different surface plasmon resonance modes. Biosensors and Bioelectronics 20, 633-642 (2004).

54 Wink, T.; Zuilen, S.J. van; Bult, A.; Bennekom, W.P., Liposome-mediated enhancement of the sensitivity in immunoassays of proteins and peptides in surface plasmon resonance spectrometry. Analytical Chemistry 70, 827-832 (1998).

55 Reather, H., Surface plasmons on smooth and rough surfaces and gratings. (Springer-Verlag, Berlin, 1988). 
56 Nelson, B.P.; Frutos, A.G.; Brochman, J.M.; Corn, R.M., Near-infrared surface plasmon resonance measurements of ultrathin films. 1 . Angle shift and SPR imaging experiments. Analytical Chemistry 71, 3928-3934 (1999).

57 Nelson, B.P.; Grimsrud, T.E.; Liles, M.R. Goodman, R.M; Corn, R.M., Surface plasmon resonance imaging measurements of DNA and RNA hybridization adsorption onto DNA microarrays. Analytical Chemistry 73, 1-7 (2001). Homola, J.; Yee, S.S.; Gauglitz, G., Surface plasmon resonance sensors: review. Sensors and Actuators B chemical 54, 3-15 (1999).

59 Cahill, D.J., Protein and antibody arrays and their medical applications. Journal of Immunological Methods 250 (1-2), 81-91 (2001).

60 Southern, E.M., Detection of specific sequences among DNA fragments separated by gel electrophoresis. Journal of Molecular Biology 98 (3), 503517 (1975).

61 Cass, T.; Ligler, F.S., Immobilized biomolecules in analysis. (Oxford University Press, Oxford, 1998).

62 TeleChem, US (2000).

63 Piner, R.D.; Zhu, J.; Xu, F.; Hong, S.; Mirking, C.A., Dip-pen nonolithography. Science 283, 661-663 (1999).

64 Liu, M.; Amro, N.A.; Liu, G., Nanografting for surface physical chemistry. the Annual Review of Physical Chemistry 59, 367-386 (2008).

65 Bernard, A.; Renault, J.P.; Michel, B.; Bosshard, H. R.; Delamarche, E, Microcontact printing of proteins. Advanced Materials 12, 1067-1070 (2000).

66 Wilkop, T.; Wang, Z.; Cheng, Q., Analysis of u-contact printed protein patterns by SPR imaging with LED light source. Langmuir 20, 11141-11148 (2004).

67 Lee, H.J.; Goodrich, T.T.; Corn, R.M., SPR imaging measurements of 1-D and 2-D DNA microarrays created from microfluidic channels on gold thin films. Analytical Chemistry 73, 5525-5531 (2001).

68 Chang-Yen, D.A.; Myszka, D.G.; Gale, B.G., A novel PDMS microfluidic spotter for fabrication of protein chips and microarrays. Journal of Microelectromechanical Systems 15 (5), 1145-1151 (2006). 
Heij, B. de; Daub, M.; Gutmann, O.; Niekrawietz, R.; Sandmaier, H.; Zengerle, R., Highly parallel dispensing of chemical and biological reagents. Analytical and Bioanalytical Chemistry 378 (1), 119-122 (2004). Maskos, U.; Southern, E.M, Oligonucleotide hybridisations on glass supports: a novel linker for oligonucleotide sysnthesis and hybridisation properties of oligonucloetides synthesised in situ. Molecular Biology 20 (7), 1679-1684 (1992).

$71 \mathrm{Yu}, \mathrm{X} . ; \mathrm{Xu}, \mathrm{D} . ;$ Cheng, Q., Label-free detection methods for protein microarrays. Proteomics 6, 5493-5503 (2006).

72 Barrett, T.; Suzek, T.O.; Troup, D.B.; Wilhite, S.E.; Ngau, W-C; Ledoux, P.; Rudnev, D.; Lash, A.E.; Fujibuchi, W.; Edgar, R., NCBI GEO: mining millions of expression profiles--database and tools. Nucleic Acids Research 33, 562-566 (2005).

73 Oron, E.; Tuller, T.; Li, L.; Rozovsky, N.; Yekutieli, D.; Rencus-Lazar, S.; Segal, D.; Chor, B.; Edgar, B.A.; Chamovitz, D.A., Genomic analysis of COP9 signalosome function in Drosophila melanogaster reveals a role in temporal regulation of gene expression. Molecular Systems Biology 3 (2007).

74 Waggoner, A., Fluorescent labels for proteomics and genomics. Current Opinion in Chemical Biology 10 (1), 62-66 (2006).

75 McDonnell, J.M., Surface plasmon resonance: towards an understanding of the mechanisms of biological molecular recognition. Current Opinion in Chemical Biology 5 (5), 572-577 (2001).

76 Day, Y.S.N.; Baird, C.L.; Rich, R.L.; Myszka, D.G., Direct comparison of binding equilibrium, thermodynamic, and rate constants determined by surface- and solution-based biophysical methods. Protein Science 11 (5), 1017-1025 (2002).

77 Langmuir, I., The adsorption of gases on plane surfaces of glass mica and platinum. Journal of the American Chemical Society. 40 (9), 1361-1403 (1918).

78 Schuck, P., Use of surface plasmon resonance to probe the equilibrium and dynamic aspects of interactions between biological macromolecules. Annual Review Biophysical Biomolecular Structure 26, 541-566 (1997). 
79 Schasfoort, R.B.M.; Tudos, A.J., Handbook of surface plasmon resonance. (The Royal Society of Chemistry, Cambridge, UK, 2008). 


\title{
3
}

\section{A soft-lithography based ligand immobilization technique}

\begin{abstract}
In this chapter a soft-lithography process to develop the various types of PDMS spotting devices for the immobilization of proteins in confined surface areas in a microarray format is explained, and various geometric examples are given. A short review of other available microfluidics spotting methods is provided, with an emphasis on PDMS based microfluidic spotting devices. PDMS based mircofluidic spotting devices are considered to be flexible and disposable, however, they are not always applicable. A spotting device merely driven on capillary forces was successfully designed, however, does not allow for high density microarrays.
\end{abstract}




\subsection{Introduction}

Microarrays consist of numerous biomolecules immobilized to a solid support in a regular pattern of microscopic spots, and have significantly evolved from the first demonstration in the 1970's, Southern blotting technique to study DNA-DNA interactions ${ }^{1}$. Labeled targets are introduced to the surface immobilized molecules, and bind or hybridize to matching ligand-analyte pairs only. The idea behind the microarray experiment is that all the ligands at the surface are exposed to the same analyte solution and can be compared without the risk of inter-experimental variations. The attempt to make arrays of DNA, RNA, peptides or proteins and to gain as much information as possible from a single test or experiment remains a huge challenge within the scientific community.

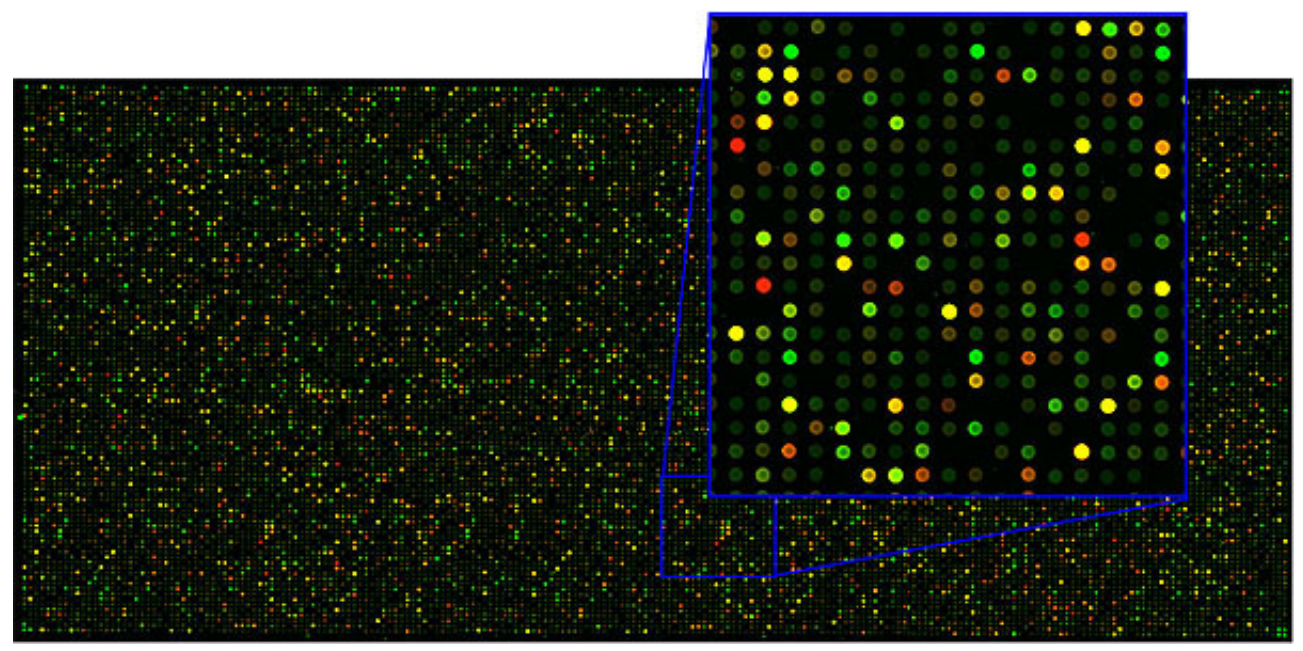

Fig. 3.1. An example of hybridization on an Agilent 60-mer oligo microarray

(http://www.rr-research.no/lothe /?k=lothe/methods\&aid=2823).

The detection technique commonly used, is based on fluorescence. Two samples, typically of a wild type and a mutant, are labeled with two different colored fluorescent dyes (often red and green). The samples are mixed, and the mixture is then introduced to the microarray, resulting in a mixture of red, green and yellow spots as can be seen in Fig. 3.1. The green spots typically indicate a down-regulation, the red spots an up-regulation and the yellow, equally regulated expression. This discrimination within one experiment can only be made by labeled detection. To obtain a similar result with a label-free detection 
technique like SPR, the two samples have to be introduced separately to the microarray, ideally one after another after a regeneration step or on a different surface containing the same microarray. The obtained binding results can then be compared directly with each other. The advantage of the label-free measurement lies within the regeneration of the surface, thereby many more samples can be compared with each other, further demonstrated in chapter 5 . The limitation, however, is the area that can be visualized at once. The fluorescence scanners can cope with much larger areas, typically a microscope slide $75 \times 25 \mathrm{~mm}$, whereas the SPR imager has a limit of $7 \times 7 \mathrm{~mm}$.

The immobilization of the molecules to the surface in this regular microarray pattern, is called spotting or printing. The various spotting techniques can be roughly divided into two groups, the contact and the non-contact spotters. The non-contact spotters deposit molecules on the surface without touching the surface. One of the most commonly used types is inkjet printing, where a droplet is ejected after compression of the air in the actuation chamber using a piezoactuator. The inkjet printing technique can also be used for in-situ synthesis of oligonucleotides by depositing molecules one base at a time at precise locations ${ }^{2}$. A more general photolithographic adsorption technique uses a photomask to synthesize the molecules in situ $^{3}$.
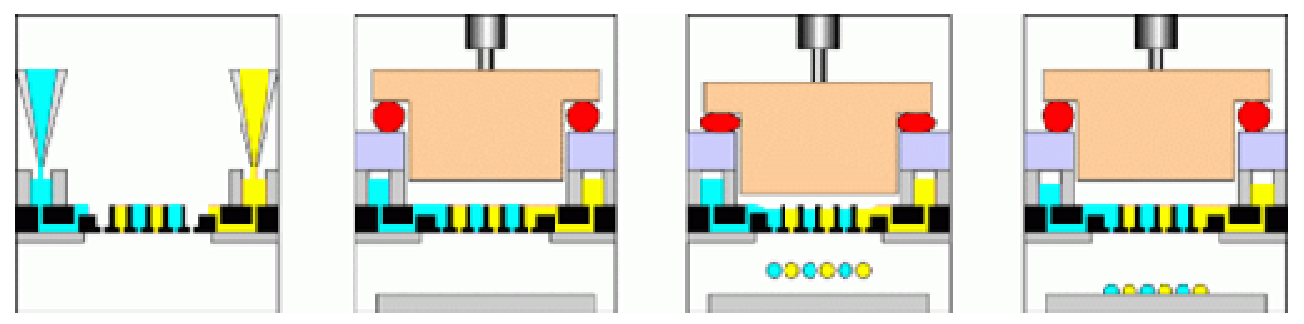

Fig. 3.2. The schematic drawing explaining the working principle of a piezoacuator ${ }^{4}$. From left to right, the capillary filling of the channels and nozzles of the printhead. The piezo stack actuator presses a stamp into the actuation chamber of the printhead. Compression of the air in the chamber results in a homogeneous build up pressure that overcomes the surface tension in the nozzles and dispensing of droplets. With the release of the pressure in the actuation chamber, the nozzles refill and are ready to print another microarray.

The contact spotters take advantage of capillary forces when releasing droplets containing molecules by touching the surface, solid or split pins are the most important. The split pin 
is mostly used as they are hollow and can take up to $1.25 \mu l^{5}$ of sample to continuously produce spots on the surface, whereas the solid pins have to be reloaded with new material after every deposition.

The piezoactuators and pin spotters are highly dependent on both, surface and fluid properties, for the obtained spot size. The need to pattern smaller surface areas made scientist look at alternatives which resulted in contact spotters based on e.g., atomic force microscope tips (AFM tips) ${ }^{6}$, microfabricated fountain pens ${ }^{7}$, or various polydimethylsiloxane (PDMS) microspotters.

PDMS is popular in the microfluidics community, because it is easy to pattern by softlithography, optically transparent, flexible, gas permeable, and low $\operatorname{cost}^{89}{ }^{10}$. Dependent on their working principle, PDMS microfluidics spotters can produce microarray with confined spotsizes regardless to the fluid or surface properties. Moreover, PDMS microfluidics spotters can facilitate a protective environment while spotting, thereby avoiding denaturating conditions while immobilizing fragile proteins and obtain more homogeneous spots, if they form a seal with the surface during the entire immobilization process. Lower ligand concentrations can be introduced and still obtain a high immobilization density due to the larger introduced volume and time.

However, PDMS it is not always applicable as it swells while absorbing organic solvents. Small molecules are also absorbed, and hydrophobic proteins stick to the walls. Its innate hydrophobic nature makes it difficult to fill narrow channels with hydrophilic fluids. And the gas permeability leads to water evaporation. There are many other materials that can be used instead of working around all the drawbacks of PDMS ${ }^{11}$. Despite the drawbacks, the use of microfluidic PDMS channels to immobilize ligands on a surface has a lot of potential, and various devices have already been reported. PDMS microstamps have been used for printing, however they are not suitable to simultaneously print numerous kinds of biosamples ${ }^{12}$. A microstamper with backfilling reservoir, which takes advantage of the surface tension for filling and releasing droplets, is a combinatory device made out of PDMS and SU-8 ${ }^{13}$. PDMS microfluidics channels, were used to make $1 \mathrm{D}$ and $2 \mathrm{D}$ patterns ${ }^{14}$ 1516 . This same principle of addressing perpendicular channels, is also used in the ProteOn XPR36 array biosensor ${ }^{17}$. The continuous flow microspotter, a pressure driven device, produces more homogeneous spots on the surface when compared to pin spotting ${ }^{18} 19$. The shape and homogeneous dispersion of the molecules within the spots is highly 
dependent on the spotting procedure, used. Undesired "donuts" are easily formed during the drying process, with the immobilized molecules located in a ring around the circumference of the spot ${ }^{20}$. The drying process is, therefore, an important and critical step in manufacturing microarrays, especially in the case of protein immobilization it is important to preserve their active state. The continuous-flow microfluidics printing devices seems to be a valid alternative for the production of homogeneous spots ${ }^{19}$.

In this chapter the various PDMS geometries are discussed to make microarrays.

\subsection{Materials and methods}

\subsubsection{Reagents}

Suberic acid bis(3-sulfo-N- hydroxysuccinimide ester) sodium salt $\left(\mathrm{BS}^{3}\right)$ were purchased from Sigma-Aldrich Chemie GmbH (Steinheim, Germany). Polyethylene glycol (PEG) diamine and 8-aminopyrene-1,3,6-trisulfonic acid trisodium salt (APTS) were purchased from Fluka Chemie GmbH (Buchs, Switzerland). Monosodium phosphate monohydrate, disodium phosphate heptahydrate and hydrochloric acid were purchased from Merck KGaA (Darmstadt, Germany). VLSI Selectipur ${ }^{\circledR}$ absolute ethanol and methanol were purchased from BASF Electronic Materials GmbH (Ludwigshafen, Germany). RBS Neutral was purchased from Chemical Products s.a. (Brussels, Belgium). Polydimethylsiloxane (PDMS) / Sylgard ${ }^{\circledR} 184$ Silicone Elastomer and Fluid 200 were purchased from Dow Corning (Midland, USA). SU-8 50 and SU-8 100 negative photoresist, Wafer P-100, size 100 $\mathrm{mm} \times 525 \mu \mathrm{m}$.

\subsubsection{TopSpot microarray fabrication}

A TopSpot device in combination with a 24-channel printhead, from BioFluidix $\mathrm{GmbH}$ (Freiburg, Germany) ${ }^{4}$, was used to spot peptides and proteins in a non-contact manner on activated SPR sensor surfaces. The principle is shown in Fig. 3.2, and the setup in Fig. 3.3. 

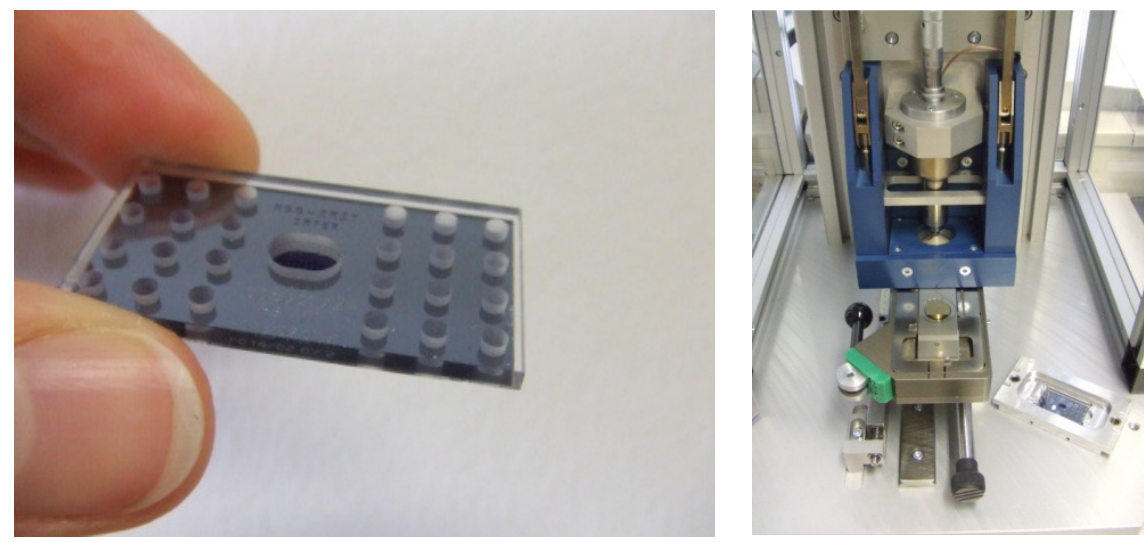

Fig. 3.3. The microfluidics chip or printhead used in combination with the TopSpot print unit.

The parameters used for actuation are, an amplitude of $55 \%$ which results with an actuation time of $6.5 \mu \mathrm{s}$. The trigger pause to refill the nozzles was set at $30 \mathrm{sec}$. Prior to spotting the actual microarray, the printhead was triggered various times to ensure a proper filling of all the channels and nozzles. After spotting, the printhead was cleaned immediately. First the excess of sample was removed from the sample reservoirs by a pipette, subsequently the printhead is placed in a cleaning station, which is placed in a glass beaker in an ultrasonic bath. The printhead is washed by flushing with a $5 \%$ RBS cleaning solution, followed by extensive flushing with Milli-Q water to prevent crosscontamination and clogging of the printhead microchannels. The clean printhead was stored in Milli-Q at $4^{\circ} \mathrm{C}$, prior to use the separate channels are flushed with Milli-Q using a $20 \mathrm{ml}$ syringe with a short rubber tube for sealing around the sample reservoirs.

The TopSpot device as shown in Fig. 3.3, is the experimental setup used for various microarray experiments in combination with SPR imaging, discussed in chapter 4, 5 and 6 . This non-contact manner of making microarrays, is working quite well for samples with similar physical properties. Furthermore, the surface hydrophilicity and solution viscosity are both parameters contributing significantly to the obtained spot size. The dense 24 spot microarray, $4 \times 6$, on top of a SPR sensor disc consists of spots of approximately $300 \mu \mathrm{m}$ in diameter and with a pitch of $500 \mu \mathrm{m}$, shown in Fig. 3.4. 


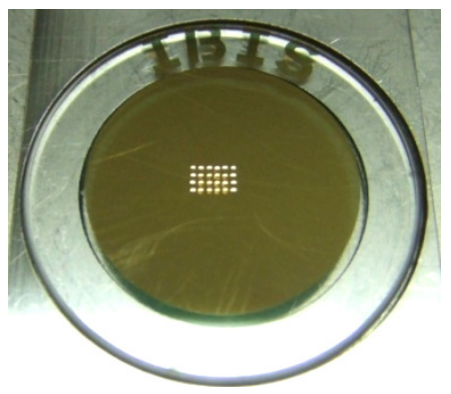

Fig. 3.4. TopSpot microarray consisting 24 spots, on a SPR sensor disc.

The microarray containing sensor is incubated in a humidity chamber at room temperature, to prevent drying of the spotted $1 \mathrm{nl}$ droplets during the 1 hour immobilization process. To avoid cross contamination of biosamples between various experiments, thorough cleaning of the printhead is essential.

\subsubsection{Soft litography}

The term soft lithography is used for the entire procedure of producing a patterned photo mask, producing a SU-8 master and finally a PDMS chip, illustrated in Fig. 3.5. The designs of the dark field chromium photo masks are made in a CAD drawing program, CleWin 3.0 (PhoeniX, Enschede, The Netherlands) and Odin. The design layouts are discussed in more detail in section 3.3.1 and 3.3.2. SU-8 photoresist, is a negative, epoxy-type, near-UV photoresist $(365 \mathrm{~nm})^{21} 22$. The mold is fabricated from two layers of SU-8. A first layer of SU-8 50 is spun on a silicon wafer at $4000 \mathrm{rpm}$ for $30 \mathrm{sec}$ resulting in a $30 \mu \mathrm{m}$ thick layer. After a soft bake at $95{ }^{\circ} \mathrm{C}$ for $>20 \mathrm{~min}$ the layer is exposed for $20 \mathrm{sec}$ with the channel mask and the second layer is spun with SU-8 100 at 500 rpm for $30 \mathrm{sec}$ resulting in a layer of $400 \mu \mathrm{m}$ thickness. In the subsequent baking step the after exposure bake of the first layer as well as the softbake of the second layer are combined at $95{ }^{\circ} \mathrm{C}$ for $30 \mathrm{~min}$. The second layer is then exposed for $120 \mathrm{sec}$ with the pillar mask. An additional after exposure bake at $95{ }^{\circ} \mathrm{C}$ for $>40 \mathrm{~min}$ is performed prior to the development of the two layers in a custom build spray development system utilizing PGMEA/RER600 developer. To remove any cracks in the developed SU-8 structures a short bake of $30 \mathrm{sec}$ at $120^{\circ} \mathrm{C}$ is performed. 


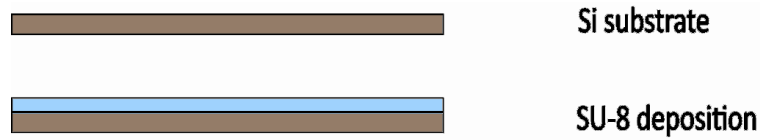

$\downarrow \downarrow \downarrow \downarrow \downarrow \downarrow \downarrow \downarrow \downarrow \downarrow \downarrow$

$------------$

UV treatment / Photo mask

Master after development of SU-8

PDMS deposition

PDMS curing

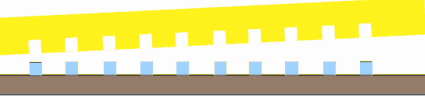

PDMS device

Fig. 3.5. Schematic representation of the various steps of soft lithography.

Polydimethylsiloxane (PDMS), a 2 phase silicone elastomer, cross links at RT after mixing the base and curing agent in a 10:1 ratio. The process is heat sensitive and curing times are reduced significantly with increasing temperature till a maximum of $150{ }^{\circ} \mathrm{C}$. Changing the ratio of the base and curing agent to $15: 1$, makes the end product more sticky. This more sticky PDMS provides a good reversible seal, applicable for some of the devices used.

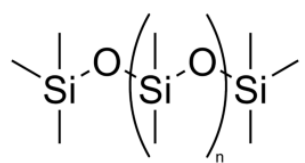

Fig. 3.6. Chemical composition of PDMS 
After weighing the base and curing agent in a disposable beaker or tube, they are mixed with a mechanical mixer. The air bubbles introduced by mixing are removed under a vacuum. The PDMS is poured carefully onto the master to avoid new air bubbles in the highly viscous solution. Dependent on the structure complexity, another degassing step can be desired. The thickness of the devices is controlled by using a special designed casting mall with spacers of various thicknesses. The PDMS, cured in an oven at $80^{\circ} \mathrm{C}$ for approximately 1 hour, is removed to make the devices. The devices are cut out by a round blade of 1 inch in diameter. The sample inlet holes are punched out by hollow needles. To ensure a proper and straight punching of the holes, a PDMS tool was developed facilitating holes on both sides

\subsection{4 $\mathrm{O}_{2}$ plasma treatment}

A reversible bond is not always desired, an irreversible bond between 2 layers of PDMS can be obtained after an oxygen plasma treatment. Both sides need to be treated for a proper bonding result. The PDMS device containing all the desired in and outlet holes was cleaned with $1: 5 \mathrm{HCl}$ in Milli-Q for 10 min at $\mathrm{RT}^{23}$, rinsed with Milli-Q and dried under a stream of nitrogen. The Tepla 300E was used in a cleanroom environment to oxidize the PDMS surface, at 110 Watt, for $1 \mathrm{~min}$, with $50 \% \mathrm{O}_{2}$. Alignment of the 2 PDMS layers was performed with a droplet of methanol and the use of a stereomicroscope. The methanol was left $\mathrm{O} / \mathrm{N}$ to evaporate and form the irreversible bond.

\subsubsection{PEG coating}

The cured, cleaned and oxidized 10:1 PDMS devices were incubated with $10 \%$ APTS in $\mathrm{dH}_{2} \mathrm{O}$ for 2 hours at RT. Then rinsed with $\mathrm{dH}_{2} \mathrm{O}$ and dried with $\mathrm{N}_{2}$, continuing with an $5 \mathrm{nM}$ $\mathrm{BS}^{3}$ in $20 \mathrm{mM} \mathrm{PO}_{4} \mathrm{pH} 7$ incubation step for $20 \mathrm{~min}$ at RT. Followed by a rinse with $20 \mathrm{mM}$ $\mathrm{PO}_{4} \mathrm{pH} 7$ and drying with $\mathrm{N}_{2}$. Finally the $1 \mathrm{nM}$ PEG in $20 \mathrm{mM} \mathrm{PO}_{4} \mathrm{pH} 7$ incubation for 30 min at RT. Another rinse with $20 \mathrm{mM} \mathrm{PO}_{4} \mathrm{pH} 7$ and $\mathrm{dH}_{2} \mathrm{O}$ before drying with $\mathrm{N}_{2}{ }^{24}{ }^{13}$. The chemical reaction scheme is illustrated in Fig. 3.7. Alignment of the structures was performed with a droplet of $\mathrm{dH}_{2} \mathrm{O}$, and left $\mathrm{O} / \mathrm{N}$ to form the irreversible bond. 


$-\mathrm{OH}$
$-\mathrm{OH}$
$-\mathrm{OH}$$\quad \mathrm{O}_{2}$ plasma treatment<smiles>CO[Si](CCN)(OC)OC</smiles>

\section{APTS treatment}

(3-aminopropyltriethoxysilane)<smiles>CO[Si](C)(OC)OCCNC(=O)CCC(=O)ON1C(=O)CCC1=O</smiles>

\section{BS3 treatment}

(bis[sulfosuccinimidyl]suberate)<smiles>CO[Si](C)(OC)OCCNC(=O)CCC(=O)NCCOCCOCCN</smiles>

PEG treatment (amino terminated polyethylene glycol)

Fig. 3.7. The reaction scheme of the PEG coating.

\subsection{Results \& discussion}

\subsubsection{PDMS array spotters}

The use of soft-lithography techniques for rapid prototyping of various PDMS spotting devices, led to the following results. Various problems arose while making and using these devices, which again led to new designs. Initially, a two layer device was used with channel and inlet hole structures in a thick PDMS layer and nozzles in a thin spincoated PDMS layer, shown in Fig. 3.8. 

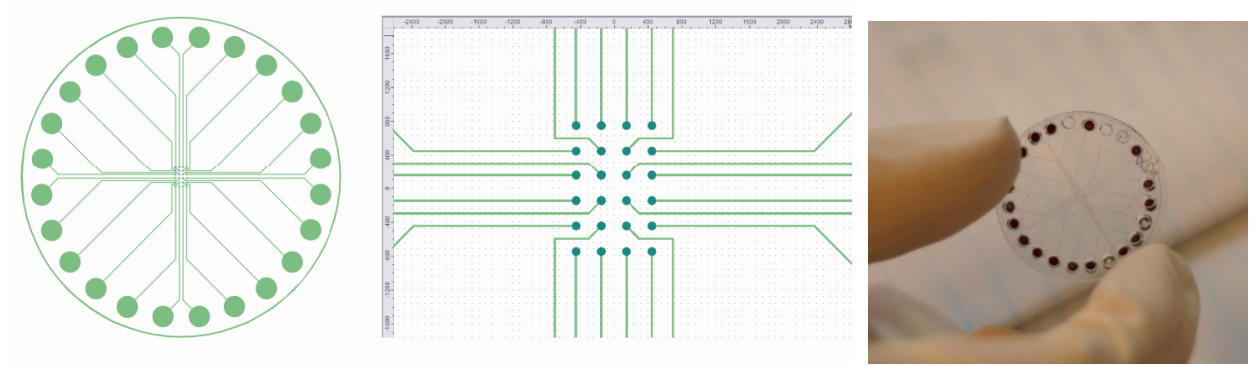

Fig. 3.8. The CleWin design of the array-spotter. The channel dimensions are, $20 \mu \mathrm{m}$ in width and $100 \mu \mathrm{m}$ in height, the nozzles at the end are $100 \mu \mathrm{m}$ long and $100 \mu \mathrm{m}$ in diameter. On the right, a device filled with ink.

Several problems arose while working with this design, firstly the bonding of the two layers of PDMS. Initially freshly prepared PDMS mixed in different ratios (10:1 and 20:1) was cured only partially and then left to cure completely after alignment to form an irreversible seal between the both PDMS layers. This was a very critical procedure, not cured enough and the PDMS devices will deform, cured too much and they will not form a good bond. An oxygen plasma treatment of the completely cured PDMS layers resulted in a strong and irreversible seal of the two PDMS layers. However, it still resulted in a poor bond around the nozzles, leading to leaking structures and cross-talk between the channels. As the combination of the high viscosity of the PDMS (4000 mPa.s) and the narrow pillar structure to obtain the nozzles, led to differences in the thickness of the spincoated PDMS layer. Decreasing the viscosity of the PDMS, with Fluid 200, initially led to a few well sealed and working devices. Secondly, the filling of the devices proved not to be without problems. In some of the devices the nozzles were blocked by a thin layer of PDMS that remained on top of the pillars. And the alignment proved to be another difficulty. Moreover, filling of the narrow hydrophobic channels was not easy without applying additional pressure. An attempt to coat the PDMS with PEG in order to increase the hydrophilicity worked out well, and can be seen in Fig. 3.9. 


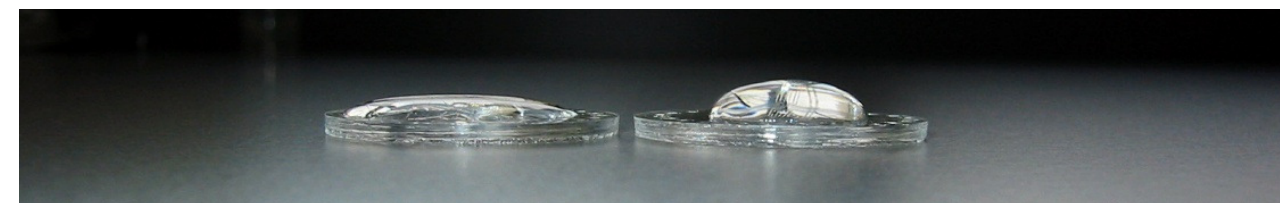

Fig. 3.9. The increased hydrophilicity of PDMS after coating with PEG visualized by the decreased contact angle of the water droplet on top of the PDMS on the left, and the untreated hydrophobic PDMS on the right.

A good bonding of the layers after coating proved to be problematic and an oxygen pasma treatment is mandatory. These coatings have to be freshly prepared due to the reattaining properties of PDMS. Due to the difficulties and additional work to obtain the coating, it was decided not to continue into this direction, and moreover the biosamples could become contaminated with the coating. Instead a new channel design was proposed to facilitate a better filling by capillary forces. This new design was made out of multiple layers of SU-8, accommodating the nozzles integrated into one design, as can be seen in Fig. 3.10 and Fig. 3.11.
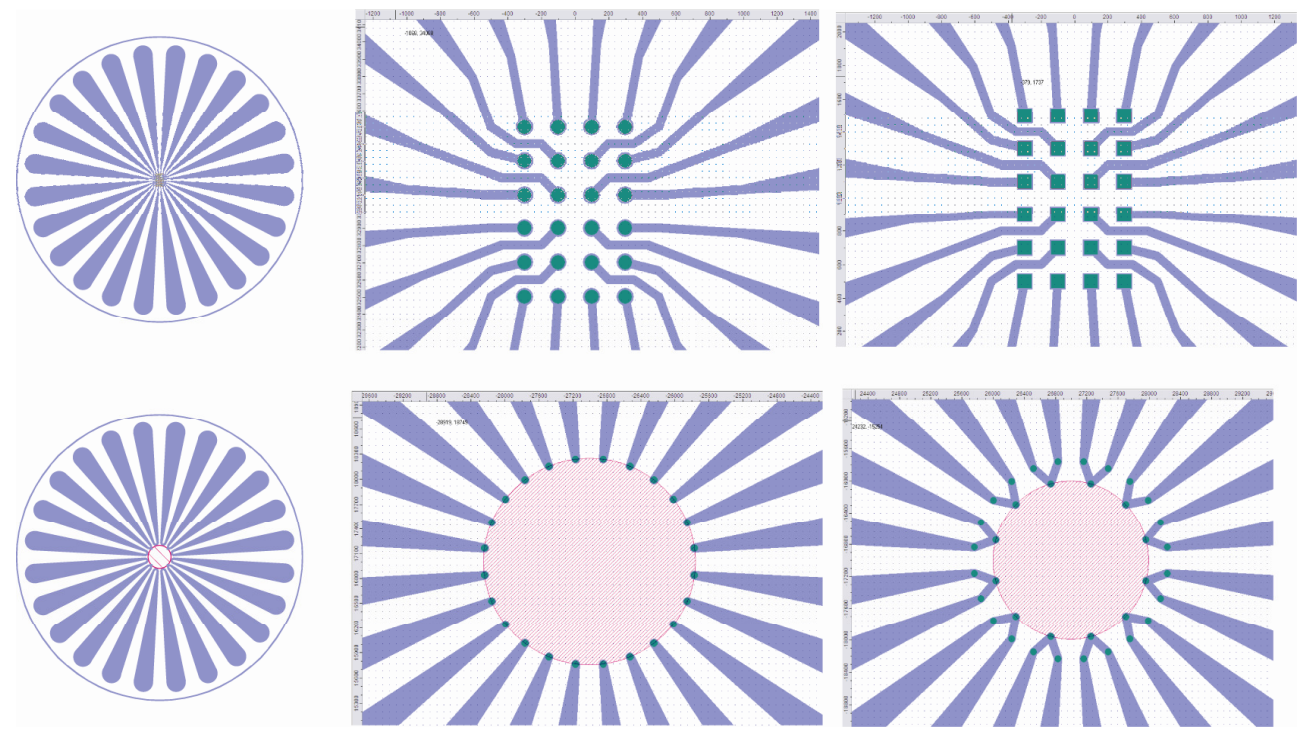

Fig. 3.10. CleWin drawings of the adjusted array- and ring-spotters. The nozzle size is $80 \mu \mathrm{m}$ in diameter. 


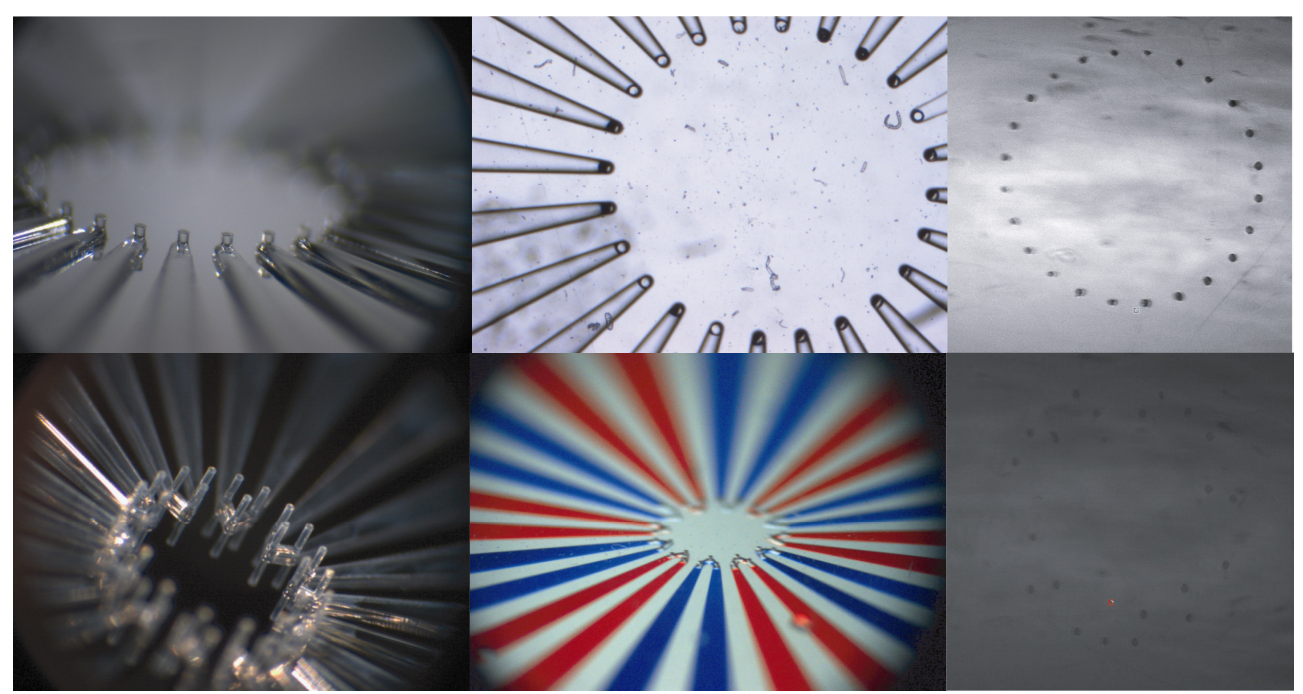

Fig. 3.11. Various images of the ring-spotters. Left, the nozzles at the end of the channels in the SU-8 maser. In the middle, light microscopy images of the PDMS ring-spotters. On the right, the spots made with the PDMS ringspotters and visualized by SPR imaging.

The resolution of the SU-8 created a problem in the case of the array-spotters, resulting in merged channels at the nozzles. The SU-8 master of the ring-spotters proved to be more fragile due to the free standing nozzles when compared to the array-spotters. The filling of the dead-end ring-spotters was not as easy as expected due to air entrapment at the end of the channel, and force needed to be applied to fill the nozzles and get the fluid in contact with the surface. The ring-spotters with the back-flow channels filled easily, however the fluid did not enter the three nozzles without applying additional pressure. Due to the fragility of the design and difficulty to fill the dead end nozzles, another design based on a flow through, or air outlet situation was proposed.

\subsubsection{PDMS line-spotters}

The PDMS line-spotters are single layered PDMS devices with open channel structures, or microslits. The initial design, shown in Fig. 3.12 and 3.13, did not contain any reservoirs and they needed to be punched out by a hollow needle. 


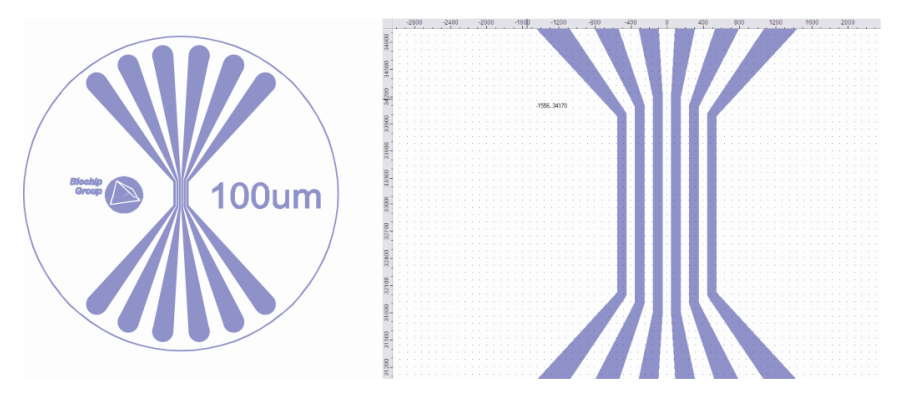

Fig. 3.12. The CleWin design of the line-spotter, the width of the channels at the narrow point is $100 \mu \mathrm{m}$, and the height is also $100 \mu \mathrm{m}$.
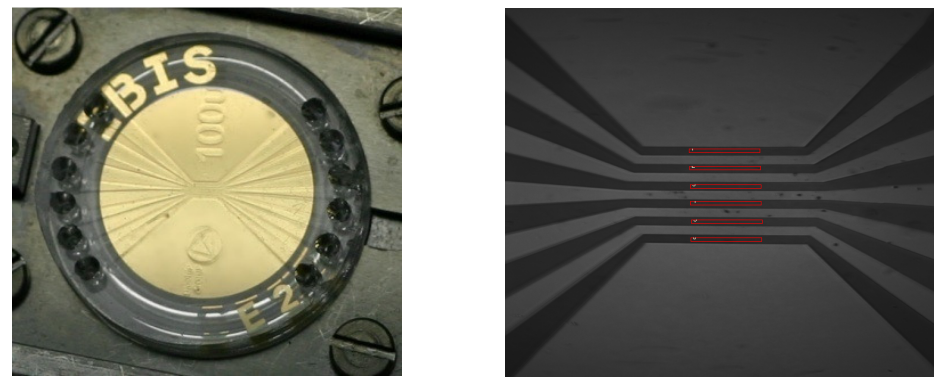

Fig. 3.13. The one dimensional immobilization of proteins on a SPR sensor surface, and the SPR image with the regions of interest (ROI's) depicted in red.

These line-spotter filled easily by capillary forces, however, additional applied pressure was sometime needed for a complete filling of the channels. Similar devices have been described before in literature, however, they all needed an oxygen plasma treatment ${ }^{14} 16$ or a differential pumping system ${ }^{15}$ to facilitate filling of the PDMS microchannels due to their geometry. The principle has even been turned into a commercially available device in another material (ProteOn, Bio Rad), here pumping is used ${ }^{17}$ to facilitate a continuous fluid flow for immobilization and measurement.

The second design of the line-spotter, shown in Fig. 3.14, is the so called stop-valve ${ }^{25}$. Here, various angles are tested to determine where the liquid pressure reaches the critical minimum to stop the capillary fluid flow at the angle transition. This design contained multiple layers (3) of SU-8 to accommodate the sample inlets and air outlets of the 126 different test structures with a channel height of $50 \mu \mathrm{m}$. 


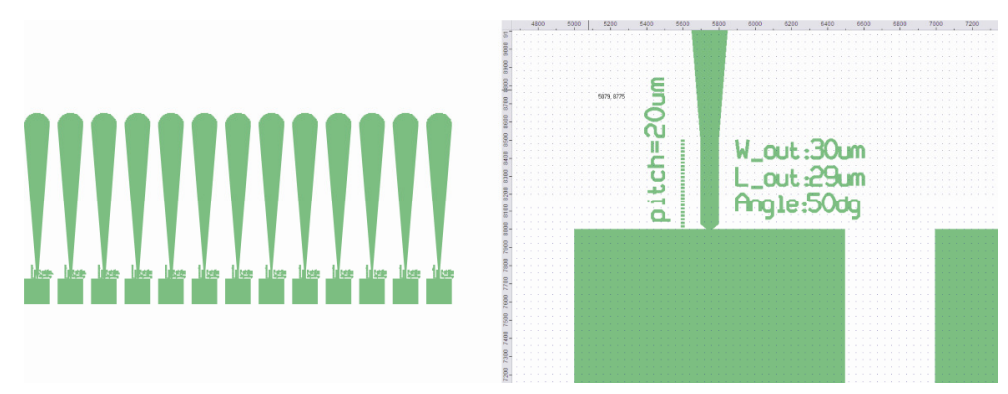

Fig. 3.14. The CleWin drawing of the stop-valve. In total 126 different designs were made to test the critical angle transition.
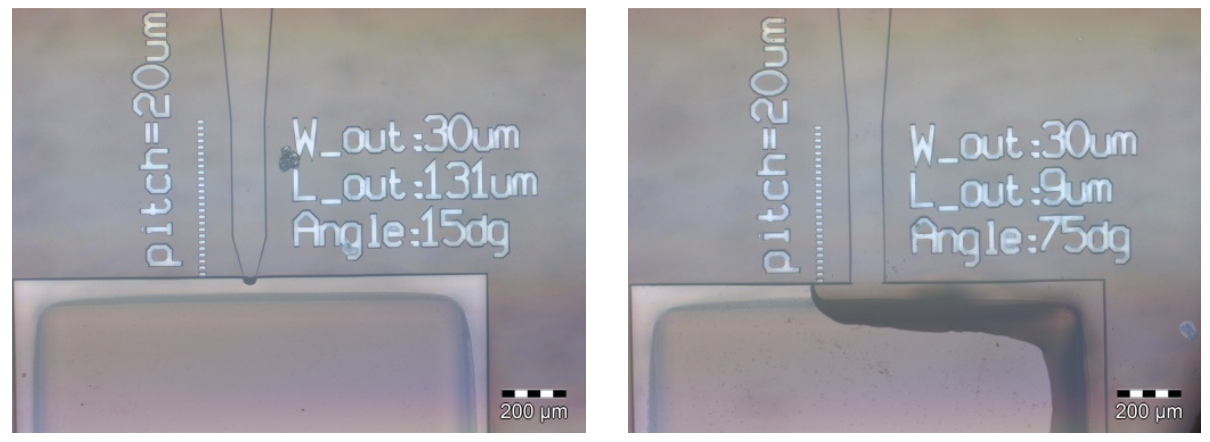

Fig. 3.15. Image of the PDMS stop-valves. Left, the fluid stopped upon angle transition with air-outlet (W_out:30 $\mu \mathrm{m} /$ Angle15dg), fluid continued into air-outlet as the angle transition was not sufficient to stop the

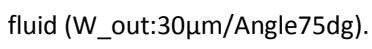

Fig. 3.15, shows an example of the stopped and continued flow in the stop-valves. Various parameters, e.g. fluid properties, channel dimensions, channel properties and transition angle are contributing to the liquid pressure and therefore the critical angle. The channel width ranges from $10 \mu \mathrm{m}$ to $90 \mu \mathrm{m}$ with interval steps of $10 \mu \mathrm{m}$, and the angle ranges from $10^{\circ}$ to $75^{\circ}$ with interval steps of $5^{\circ}$. Comparison of the results led to the following findings, the flow stops at an angle transition of $10^{\circ}$ to $60^{\circ}$ when the width ranges from 10 $\mu \mathrm{m}$ till $40 \mu \mathrm{m}$. With a width of $50 \mu \mathrm{m}$ to $80 \mu \mathrm{m}$, the critical angle transition changed to $35^{\circ}$ $\pm 5^{\circ}$. The $90 \mu \mathrm{m}$ width structures were never able to stop the flow, due to the geometry the angle did not affect the flow enough. Due to the geometry of the stop-valves, these are the first PDMS based devices to only fill by capillary forces without any additives to change or overcome the hydrophobic nature of PDMS. The wide sample inlet and conical shaped channel provided a good fluid flow driven by capillary forces, and the air outlet at 
the end of the channel resulted in an easier and faster filling. A continuous flow similar to the line-spotter, is not possible without applying pressure to overcome the pressure drop caused by the stop-valve.

Stop-valves to immobilize ligands at a SPR sensor surface, arranged in such a way that they would fit in the flow-cell, have not been made so far. As a straight, parallel organization of the structures would only lead to 6 different ligands to be immobilized in the flow-cell. As the inlet size of the stop-valve is $1.5 \mathrm{~mm}$ in diameter, with a pitch of $2 \mathrm{~mm}$, and the usable flow-cell area $\approx 5 \mathrm{~mm}$ in length. Rearrangement of the air-outlet could lead to 24 structures placed in a ring, similar to the ring-spotters. However, this would still not lead to high density microarrays, therefore, other techniques based on pressure driven flows are needed $^{19}$.

Observations with ethanol showed none of the angles were able to stop the fluid flow. Fluids with other viscosities or surface tensions has not been tested, but it can be assumed that the critical angle will change according to the fluid properties. Also the surface characteristics influence the critical angle, but have also not been investigated here 2627 . Besides the flow control, another possible application could be surface hydrophobicity measurements or sample concentration measurements based on the critical angle, but an extensive study needs to be performed in order to make that statement. Our interest was to obtain a high density microarray to study binding of (auto)antibodies at a SPR sensor surface, which turned out to be unsuccessful due to experimental limitations, therefore we decided to proceed with the commercially available piezoactuator.

\subsection{Conclusion}

The various soft-lithography based ligand immobilization techniques, PDMS microfluidics spotting devices, described in this chapter all had their advantages and disadvantages. The narrow channel devices, allow high density microarray structures, however, also need a pressure to fill the hydrophobic channels. An attempt to make a soft-lithography based ligand immobilization technique merely driven by capillary forces resulted in a successful device, the stop-valve. Because it is a microslit structure, one of the most important limiting parameters is the size of the surface, in our case the 1 inch in diameter SPR sensor surface. Also the flow-cell dimensions $(3 \times 11 \mathrm{~mm})$ to address the analytes to the 
microarray area is important for the design of the spotting device. In general the geometry of the stop-valves does not allow for high density microarrays, because the channels are too spacious.

The PDMS based spotting devices, seem to be an interesting and disposable alternative for immobilizing ligands at the surface. Especially the confined and smaller spot sizes with increased ligand density and homogeneity are valuable improvements over the commonly used spotting techniques. However, one should keep in mind that PDMS is not applicable for all (bio)samples, as it adsorbs small molecules and it is very hydrophobic by nature. Alternative materials or surface coatings could to be investigated to obtain a more suitable alternative spotting technique. Because of other interests, we decided not to proceed further in this direction and used the commercially available TopSpot instead, to obtain fast results. Even though the TopSpot has its own limitations, the results we obtained with the peptide microarrays were very satisfactory and are further discussed in the following three chapters.

\subsection{References}

1 Southern, E.M., Detection of specific sequences among DNA fragments separated by gel electrophoresis. Journal of Molecular Biology 98 (3), 503517 (1975)

2 Maskos, U.; Southern, E.M, Oligonucleotide hybridisations on glass supports: a novel linker for oligonucleotide sysnthesis and hybridisation properties of oligonucloetides synthesised in situ. Molecular Biology 20 (7), 1679-1684 (1992).

3 Gao, X.; Zhou, X.; Gulari, E., Light directed massively parallel on-chip synthesis of peptide arrays with t-Boc chemistry. Proteomics 3, 2135-2141 (2003).

4 de Heij, B.; Daub, M.; Gutmann, O.; Niekrawietz, R.; Sandmaier, H.; Zengerle, R., Highly parallel dispensing of chemical and biological reagents. Analytical and Bioanalytical Chemistry 378 (1), 119-122 (2004).

5 TeleChem, US (2000). 
6 Piner, R.D.; Zhu, J.; Xu, F.; Hong, S.; Mirking, C.A., Dip-pen nonolithography. Science 283, 661-663 (1999).

7 Reese, M.O.; van Dam, R.M.; Scherer, A.; Quake, S.R., Microfabricated Fountain Pens for High-Density DNA Arrays. Genome Research 13 (10), 23482352 (2003).

8 Younan Xia, George M. Whitesides, Soft Lithography. Angewandte Chemie International Edition 37 (5), 550 (1998).

9 Anderson, J.R.; Chiu, D.T.; Jackman, R.J.; Cherniavskaya, O.; McDonald, J.C.; Wu, H.; Whitesides, S.H.; Whitesides, G.M., Fabrication of Topologically Complex Three-Dimensional Microfluidic Systems in PDMS by Rapid Prototyping. Analytical Chemistry 72 (14), 3158-3164 (2000).

10 Thorsen, T.; Maerkl, S.J.; Quake, S.R., Microfluidic Large-Scale Integration. Science 298 (5593), 580-584 (2002).

11 Mukhopadhyay, R., When PDMS isn't the best. Analytical Chemistry, 32493253 (2007).

12 Bernard, A.; Renault, J.P.; Michel, B.; Bosshard, H.R.; Delamarche, E, Microcontact printing of proteins. Advanced Materials 12, 1067-1070 (2000).

13 Lin, S.C.; Tseng, F.G.; Huang, H.M.; Chen, Yu-Feng; Tsai, Y.C.; Ho, C.E.; Chieng, C.C., Simultaneous immobilization of protein microarrays by a micro stamper with back-filling reservoir. Sensors and Actuators B: Chemical 99 (1), 174-185 (2004).

14 Delamarche, E.; Bernard, A.; Schmid, H.; Michel, B.; Biebuyck, H.;, Patterned Delivery of Immunoglobulins to Surfaces Using Microfluidic Networks. Science 276 (5313), 779-781 (1997).

15 Lee, H.J.; Goodrich, T.T.; Corn, R.M., SPR imaging measurements of 1-D and 2-D DNA microarrays created from microfluidic channels on gold thin films. Analytical Chemistry 73, 5525-5531 (2001).

16 He, X., Dandy, D.S., and Henry, C.S., Microfluidic protein patterning on silicon nitride using solvent-extracted poly(dimethylsiloxane) channels. Sensors and Actuators B: Chemical 129 (2), 811-817 (2008).

17 Bravman, T.; Bronner, V.; Lavie, K.; Notcovich, A.; Papalia, G.A.; Myszka, D.G., Exploring "one-shot' kinetics and small molecule analysis using the ProteOn XPR36 array biosensor. Analytical Biochemistry 358, 281-288 (2006). 
Chang-Yen, D.A.; Myszka, D.G.; Gale, B.G., A novel PDMS microfluidic spotter for fabrication of protein chips and microarrays. Journal of Microelectromechanical Systems 15 (5), 1145-1151 (2006). Natarajan, S.; Katsamba, P.S.; Miles, A.; Eckman, J.; Papalia, G.A.; Rich, R.L.; Gale, B.K.; Myszka, D.G., Continuous-flow microfluidic printing of proteins for array-based applications including surface plasmon resonance imaging. Analytical Biochemistry 373 (1), 141-146 (2008). Kim, H.Y.; Lee, S.E.; Kim, M.J.; Han, J.I.; Kim, B.K.; Lee, Y.S.; Lee, Y.S.; Kim, J.H., Characterization and simulation of cDNA microarray spots using a novel mathematical model. BMC Bioinformatics 8 (1), 485 (2007).

21 Lorenz, H., Despont, M., Fahmi, N., LaBianca, N., Renaud, P., Vettiger, P., SU8: a low-cost negative resist for MEMS. Journal of Micromechanichs and Microengineering 7, 121-124 (1997).

22 Campo, A. del, Greiner, C., SU-8: a photoresist for high-aspect-ratio and 3D submicron lithography. Journal of Micromechanichs and Microengineering 17, 81-95 (2007).

23 Jo, B.H.; Van Lerberghe, L.M.; Motsegood, K.M.; Beebe, D.J. ThreeDimensional Micro-Channel Fabrication in Polydimethylsiloxane (PDMS) Elastomer. Journal of Microelectromechanical Systems 9 (1), 76 (2000).

Donzel, C.; Geissler, M.; Bernard, A.; Wolf, H.; Michel, B.; Hilborn, J.; Delamarche, E.; Hydrophilic Poly(dimethylsiloxane) Stamps for Microcontact Printing. Advanced Materials 13 (15), 1164-1167 (2001).

25 Man, P.F.; Mastrangelo, C.H.; Burns, M.A.; Burke, D.T., presented at the IEEE Micro Electrical Mechanical Systems MEMS, Heidelberg, 1998 (unpublished).

26 Melin, J.; Roxhed, N.; Gimenez, G.; Griss, P.; Wijngaart, W. van der; Stemme, G., A liquid-triggered liquid microvalve for on-chip flow control. Sensors and Actuators B chemical 100, 463 (2004).

27 Feng, Y.; Zhou, Z.; Ye, X.; Xiong, J.; Passive valves based on hydrophobic microfluidics. sensors and actuators A physical 108, 138 (2003). 



\title{
$4 \quad$ Angle-scanning SPR imaging for detection of biomolecular interactions on microarrays
}

\begin{abstract}
In this chapter we describe the use of a commercial surface plasmon resonance (SPR) imaging instrument for monitoring the binding of biomolecules on user-defined regions of interest of a microarray. By monitoring the angle shift of the SPR-dip using a continuous angle-scanning mode instead of monitoring the change in reflectivity at a fixed angle, a linear relationship with respect to the mass density change on the surface will remain over a wide dynamic angle range of $8^{\circ}$. Peptides (2.4 kDa) and proteins (150 kDa) were both spotted on the same sensor chip to illustrate that both, low and high molecular weight ligands with initial large differences in off-set SPR angles, can be applied within the same experiment. By using a fluorescently labeled antibody, SPR results can be confirmed by means of fluorescence microscopy after completion of a SPR experiment. SPR imaging in angle-scanning operation provides sensitive, accurate, and label-free detection of analyte binding on microarrays containing different molecular weight ligands.
\end{abstract}

Biosensors and Bioelectronics 23 (2008) 839-844. 


\subsection{Introduction}

Surface plasmon resonance (SPR) biosensors enable today's research to explore the kinetics of biomolecular interactions ${ }^{1}$. The use of SPR for biosensing, first mentioned in the literature in the $1980 \mathrm{~s}^{2}{ }^{3}$, allows label-free and real-time detection of various biomolecular interactions 456 . Many different SPR platforms and combinations of technologies have been described ${ }^{7}$ : e.g. SPR imaging ${ }^{89}{ }^{10}$, SPR microscopy ${ }^{11}{ }^{12}$, surface plasmon field-enhanced fluorescence spectroscopy (SPFS) ${ }^{13}{ }^{14}$, scanning electrochemical microscopy-SPR (SECMSPR) ${ }^{15}$, grating-coupled SPR (GC-SPR) 161718 and waveguidecoupled SPR (WCSPR) ${ }^{19}$. The SPR principle is based on the excitation of surface plasmons in a thin layer $(\approx 50 \mathrm{~nm}$ ) of a metal such as gold, using p-polarized light. At a certain angle of incidence, the free electron oscillation in gold reaches a maximum and an evanescent field is being enhanced by at least a factor of $30^{203}$ along the liquid-gold interface. The actual angle of the so-called SPR-dip depends on the wavelength of the used light and the refractive indexes of the layers, e.g. prism, gold and liquid layer. Analyte binding onto the sensor surface will induce a change of the refractive index at the gold-liquid interface, resulting in an angle shift of the SPR-dip. SPR measurements carried out in a fixed angle mode often translate the angle shift of the SPR-dip in a reflectivity signal calculated from the linear part of the SPR-curve ${ }^{21}$. These reflectivity signals show linearity up to $5 \%$ change in reflectivity ${ }^{22}$, which is equal to a $50 \mathrm{~m}^{\circ}$ angle shift of the SPR-dip. The actual shift in SPR-dip or the derived parameter (change in reflectivity) followed as a function of time is plotted in a so-called sensorgram. The most-common geometries of SPR platforms are in the socalled Kretschmann configuration ${ }^{23}$, in which the incident light comes from the high refractive index medium (prism) and reflects at the gold surface without traveling through the liquid. In grating-coupled SPR setups ${ }^{16} 17$, the incident light passes through the liquid before reflecting at the grating, and therefore sample solution and flow-cell need to be optically transparent ${ }^{24}$. Biacore $A B$ (Uppsala, Sweden) has multiple SPR systems to analyze protein interactions based on the Kretschmann configuration 2526 . However, the number of sensor spots in these instruments is limited to 20 in the A100 Biacore system ${ }^{27}$. With the FLEXChip, a grating-coupled SPR imaging system and hence with the drawback that the optical path is through the sample solution, it is possible to analyze 400 interactions simultaneously ${ }^{17}$. In this paper we describe a new commercial SPR imaging system in the Kretschmann configuration for imaging a $50 \mathrm{~mm}^{2}$ gold surface. In this system, biomolecular interactions are detected by monitoring the angle shift of the 
SPR-dip using a continuous angle-scanning mode. In principle more than 500 different regions of interest (ROIs) can be defined and therefore more than 500 individual SPR dips can be measured to follow the actual SPR angles from the imaged sensor surface, enabling simultaneous measurement of multiple biomolecular interactions.

\subsection{Materials and methods}

\subsubsection{Reagents}

1-Ethyl-3-(3-dimethylaminopropyl)carbodiimide (EDC), N-hydroxysuccinimide (NHS), ethanolamine, phosphate buffered saline (PBS), glycine. $\mathrm{HCl}$ and human IgG were purchased from Sigma-Aldrich Chemie GmbH (Steinheim, Germany). Glycerol and Tween 20 were purchased from Fluka Chemie $\mathrm{GmbH}$ (Buchs, Switzerland). Alexa Fluor ${ }^{\circledR} 488$ labeled mouse anti-biotin IgG and biotin-XX goat anti-rabbit IgG $(H+L)$ were purchased from Molecular Probes/Invitrogen (Breda, The Netherlands). Morpholinoethanesulfonic acid (MES) monohydrate and acetic acid were purchased from Merck KGaA (Darmstadt, Germany). Refractive index matching liquid $n=1.518 \pm 0.0002$ and $n=1.7200 \pm 0.0005$ from R.P. Cargille Laboratories Inc. (Cedar Grove, USA). VLSI Selectipur ${ }^{\circledR}$ absolute ethanol was purchased from BASF Electronic Materials $\mathrm{GmbH}$ (Ludwigshafen, Germany), Assistend $^{\circledR}$ optical paper was purchased from Omnilabo International B.V. (Breda, The Netherlands) and RBS Neutral was purchased from Chemical Products s.a. (Brussels, Belgium). All buffer solutions were prepared with Milli-Q water.

\subsubsection{SPR setup}

The imaging surface plasmon resonance instrument for biomolecular interaction sensing (iSPR-IBIS) from IBIS Technologies B.V. (Hengelo, The Netherlands) constitutes a flexible SPR imaging platform with an imaged area of about $50(7 \times 7) \mathrm{mm}^{2}$ with a minimal spot-tospot distance of $15 \mu \mathrm{m}$. In the iSPR, the light is reflected on an angle-controlled mirror (range $8^{\circ}$ ) before passing through a hemispherical prism to enable fast scanning of the angle shift of the SPR-dip of the individual ROls. A schematic drawing of the iSPR optical setup is shown in Fig. 4.1. 


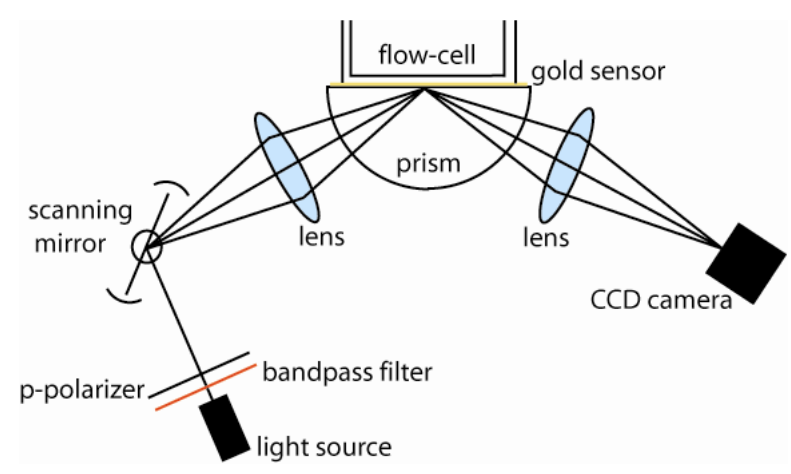

Fig. 4.1. Schematic representation of the optical configuration of the iSPR. The white light passes through a bandpass filter to set the wavelength of the incident light at $840 \mathrm{~nm}$, before passing through a p-polarizer. The incident light is being reflected by the angle controlled mirror with a maximal scanning angle of $8^{\circ}$, and focused by a lens before reaching the hemispheric BK7 prism with a refractive index of $n=1.518$ and $r=13 \mathrm{~mm}$. Dependent on the application the Au surface can be coated with functionalized groups accordingly. The reflected light is passed through another focusing lens before the 12 bits CCD camera captures at least 10 images per second which will be analyzed in real-time by the software.

The SPR angle range can be optimized by manual adjustment of the optical leverage arm, between $66^{\circ}$ of $78^{\circ}$. A bare gold sensor disc (IBIS Technologies) was placed carefully on a clean hemispheric BK7 prism using a droplet of refractive index matching liquid $(n=1.518)$ to avoid entrapment of air-bubbles. Different types of flow-cells or mixing cuvette-based systems are available for addressing the sample over the sensor surface in the iSPR. A schematic drawing of the liquid handling system of the iSPR including flow-cell is shown in Fig. 4.2. A $3 \mu \mathrm{l}$ flow-cell, with chamber dimensions of $10 \mathrm{~mm} \times 3 \mathrm{~mm} \times 0.1 \mathrm{~mm}(\mathrm{I} \times \mathrm{w} \times \mathrm{h})$, is placed over a 24 spot microarray and sealed with a rubber O-ring by fixing the screws in the sample rack. The temperature of the sample is controlled by two independently controlled Peltier elements, one located in the sample rack and the other one in high precision $\left(<0.01^{\circ} \mathrm{C}\right)$ around the flow-cell, liquid handling procedures (LHPs) were written in the iSPRsoftware to navigate the liquid-handler and thereby facilitating a fully automated performance of the experiment. To reduce the amount of sample needed during the interaction time, a sample plug was guided over the sensor surface by repeated changing of the flow direction. Depending on the type of interaction sample volumes can range from 125 to $400 \mu \mathrm{l}$, with a mixing (back and forth) volume of 50-300 $\mu \mathrm{l}$ and speed of $2 \mu \mathrm{l} / \mathrm{s}$. A transient response of $2 \mathrm{~m}^{\circ}$ is the result of the changing flow direction. 


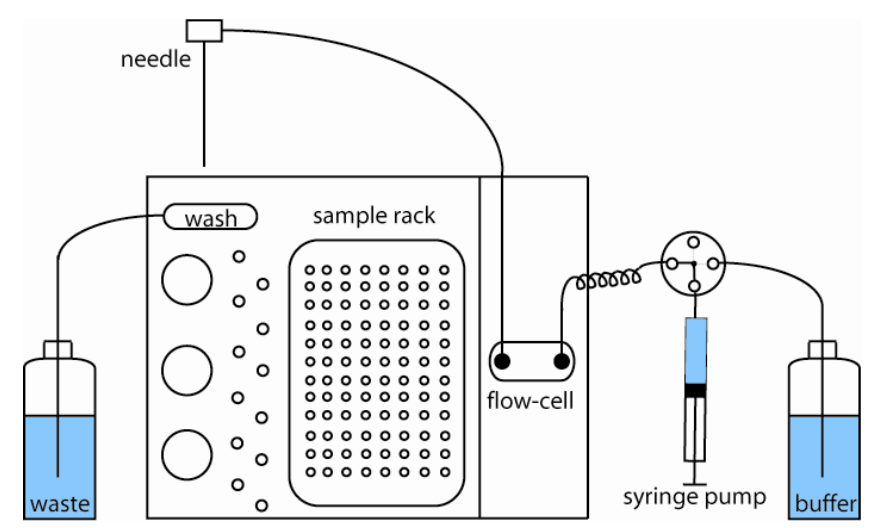

Fig. 4.2. Schematic representation of the liquid handling system of the iSPR. Samples can be collected from several containers in the Peltier controlled thermostatted sample rack, e.g. $3 \times 10 \mathrm{ml}$ containers, $12 \times 0.5 \mathrm{ml}$ tubes or a microtiter plate, using the needle at the end of the liquid handler robot arm which is connected to the flow-cell, which is separately thermostatted in high precision. The mixing function of the $1 \mathrm{ml}$ syringe pump facilitates a continuous back- and forward flow of the sample over the sensor surface and thereby reducing the total amount of sample needed. The sample is being washed away with buffer with the needle positioned in the wash station.

The SPRimager ${ }^{\circledR}$ II from GWC Technologies (Madison, WI, USA), a fixed angle mode instrument was used for comparison ${ }^{21}$. A bare gold sensor disc (SPRchip ${ }^{\mathrm{TM}}$, GWC Technologies) was placed carefully on a SF10 prism using a droplet of refractive index matching liquid $(n=1.7200)$. The standard flow-cell configuration with chamber dimensions of $0.5 \mathrm{~mm} \times 13 \mathrm{~mm}$ ( $\mathrm{h} \times$ diameter) used a minimum of $1 \mathrm{ml}$ sample when recirculated by the peristaltic pump.

\subsubsection{Refractive index determination}

The refractive indexes $(\Delta n)$ of glycerol dilution series, from 0 to $25 \%$ in PBS/0.03 \% Tween 20 (PBST), were determined by an analog laboratory refractometer (\#354636, Carl Zeiss/JENA, Germany) at $20^{\circ} \mathrm{C}$. Afterwards these glycerol dilutions were used to determine the angle shift using the iSPR in combination with a bare gold sensor disc from IBIS Technologies B.V. (Hengelo, The Netherlands) and the reflectivity change using the SPRimager ${ }^{\circledR} I I$ in combination with a bare gold sensor disc from GWC Technologies. Both SPR measurements were performed at $20^{\circ} \mathrm{C}$. 


\subsubsection{Microarray fabrication}

A HC200m sensor disc from XanTec Bioanalytics GmbH (Muenster, Germany), functionalized with a polycarboxylated hydrogel of $200 \mathrm{~nm}$ thickness and with medium density, was activated by shaking for $20 \mathrm{~min}$ at room temperature with $0.4 \mathrm{M} \mathrm{EDC/0.1} \mathrm{M}$ NHS in MES buffer (50 mM, pH 5.4) ${ }^{28} 29$. The activated surface was rinsed with acetic acid ( $50 \mathrm{mM}, \mathrm{pH}$ 3.0) and dried thoroughly for 5 min by applying a 2 bar flow of nitrogen. $A$ Topspot device in combination with a 24-channel printhead, from BioFluidix $\mathrm{GmbH}$ (Freiburg, Germany) ${ }^{30}$, was used to spot the peptides and proteins in a non-contact manner on the sensor surface (layout of the array is described in the caption of Fig. 4.7a). Between spotting runs the printhead was cleaned according to a protocol from BioFluidix with a $5 \%$ RBS cleaning solution and Milli-Q water to prevent cross-contamination. The 24 spot microarray, $4 \times 6$, on top of the sensor disc consists of spots of approximately $200 \mu \mathrm{m}$ in diameter and with a pitch of $500 \mu \mathrm{m}$. The sensor was incubated in a humidity chamber at room temperature, to prevent drying of the spotted $1 \mathrm{nl}$ droplets during the 1 hour immobilization process. After immobilization, the remaining active groups of the sensor surface were quenched with ethanolamine $(1 \mathrm{M}, \mathrm{pH} 8.0)$ for $10 \mathrm{~min}$ at room temperature. The sensor was stored in PBS, $\mathrm{pH} 7.4$, before the measurement to prevent drying of the array.

\subsubsection{Detection of biomolecular interactions on the peptide/antibody microarray}

The sensor containing the peptide/antibody microarray was mounted in the iSPR and equilibrated using $1 \mathrm{ml}$ PBST in the flow-cell at a flow-speed of $2 \mu \mathrm{l} / \mathrm{s}$ at $24^{\circ} \mathrm{C}$. After defining the ROls of $150 \mu \mathrm{m} \times 150 \mu \mathrm{m}$, the SPR-dip was being determined automatically by the software. The flow-speed during the interactions was kept at $2 \mu \mathrm{l} / \mathrm{s}$, using a $50 \mu \mathrm{l}$ mixing volume. A baseline was made in PBST followed by 1 hour interactions with varying anti-biotin IgG concentrations. Signals were determined as the angle-shift between PBST wash-step after interaction and the PBST baseline before the interaction. The sensor was regenerated with $10 \mathrm{mM}$ glycine $\cdot \mathrm{HCl}, \mathrm{pH} 1.5(500 \mu \mathrm{l}, 20 \mu \mathrm{l} / \mathrm{s})$ between the various interactions. The data analysis was performed with ISPRAD software from IBIS Technologies B.V. (Hengelo, The Netherlands). 


\subsubsection{Fluorescence microscopy}

After completion of the SPR experiment, the sensor disc was removed from the hemisphere and carefully placed on a microscope slide with the interaction side facing down and a droplet of PBST in between. The inverted Olympus IX51 microscope was used for analysis, with an Olympus U-MWB2 filter in combination with a mercury lamp. Images were taken with a ColorViewII CCD camera and analysis ${ }^{\circledR}$ FIVE software from Olympus Soft Imaging Solutions GmbH (Muenster, Germany).

\subsection{Results and discussion}

\subsubsection{SPR linearity}

In order to apply SPR imaging for quantitative adsorption experiments, it is important to have a linear correlation between the changes in reflectivity $(\Delta \% R)$ and changes in the refractive index ( $\Delta n$, amount of adsorbed molecules on the surface). SPR systems measuring at a fixed angle positioned near to the inflection point of the left-flank of the SPR curve, can only apply the small linear relationship between reflectivity and mass change for reflectivity changes of less than $5 \%\left(\text { or } 50 \mathrm{~m}^{\circ}\right)^{22}$ due to the shape of the SPR curve. The linear correlation between the angle-shift of the SPR-dip and the change in refractive index was tested by varying concentrations of glycerol in PBST (w/v) in the continuous angle-scanning mode of the iSPR system (IBIS) and the fixed angle mode of the SPRimager ${ }^{\circledR}||$ (GWC). The repeatability was tested by performing the experiments three times on new sensor discs; the obtained relative standard deviation obtained was always less than 0.5 units. The refractive indexes of glycerol samples $\leq 0.05 \%$ were calculated as they could not be measured with the refractive index meter used. Fig. 4.3 shows the correlation between the angle-shift/the reflectivity and the refractive index change of the various glycerol concentrations. The obtained linearity between angle shift and refractive index with the angle-scanning mode indicates a dynamic range of more than $2^{\circ}$ corresponding to a refractive index change $\Delta n \geq 0.03$. Our studies also showed a linearity up to $25 \%$ in the fixed angle mode, contrary to the $5 \%$ found in the literature ${ }^{22}$. The dynamic range of the scanning angle mode is 10 times larger compared to the fixed angle mode, when the ability to measure the change of refractive index is taken into account. 
The maximal angle-scanning range of the iSPR is $8^{\circ}$; which would theoretically correspond with a maximum refractive index change of 0.1 . However, with the prism used (BK7), SPR dips cannot be found at those high refractive indexes as the necessary incident angle exceeds the mechanical capacity of the leverage. A change to SF10 glass, which has a higher refractive index (1.710) compared to BK7 (1.510), should provide the possibility to measure refractive index changes up to 1.6, as was calculated by applying a 2 phase Fresnel reflectivity model ${ }^{31}$.

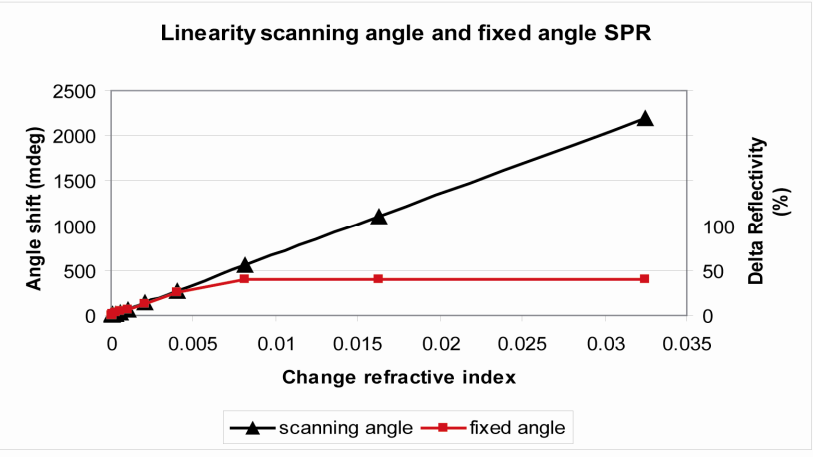

Fig. 4.3. Relationship between the angle shift of the SPR-dip $\left(\mathrm{m}^{\circ}\right)$ and the change in refractive index, in the scanning angle mode, and relationship between the delta reflectivity and the change in refractive index in the fixed angle mode. Twelve serial dilutions: $0.0,0.025,0.05,0.1,0.2,0.4,0.8,1.6,3.2,6.25,12.5$ and $25 \%$ glycerol in PBST were used. The scanning angle SPR measurements (black line) show an increased linear dynamic range with a correlation coefficient of 1 throughout the measurement compared to the fixed angle SPR measurements (red line) with a correlation coefficient of 0.998 for the refractive index changes up to 0.004 .

\subsubsection{Limit of detection}

In order to give an indication of the limit of detection (LOD) and the dynamic range of a biomolecular system, a microarray containing various concentrations of ligands with different molecular weights was spotted on a sensor disc. A low molecular weight biotinylated peptide (2.4 kDa) and biotin-XX goat anti-rabbit IgG $(\mathrm{H}+\mathrm{L})(150 \mathrm{kDa})$ were spotted on the same sensor chip. To be able to verify the biomolecular interaction with a fluorescence measurement, Alexa Fluor ${ }^{\circledR} 488$ labeled mouse anti-biotin IgG (150 kDa) was applied. The anti-biotin antibody will bind to both the biotinylated groups of the peptide and the IgG. Regeneration of the sensor with $10 \mathrm{mM}$ glycine $\cdot \mathrm{HCl}, \mathrm{pH} 1.5$ provided the 
ability to test several antibody concentrations on one sensor disc. Fig. 4.4 shows the sensorgram of the interaction of the biotinylated peptides and -proteins with the antibiotin antibody. The angle shift $\left(\mathrm{m}^{\circ}\right)$ is shown as a function of time $(\mathrm{s})$.

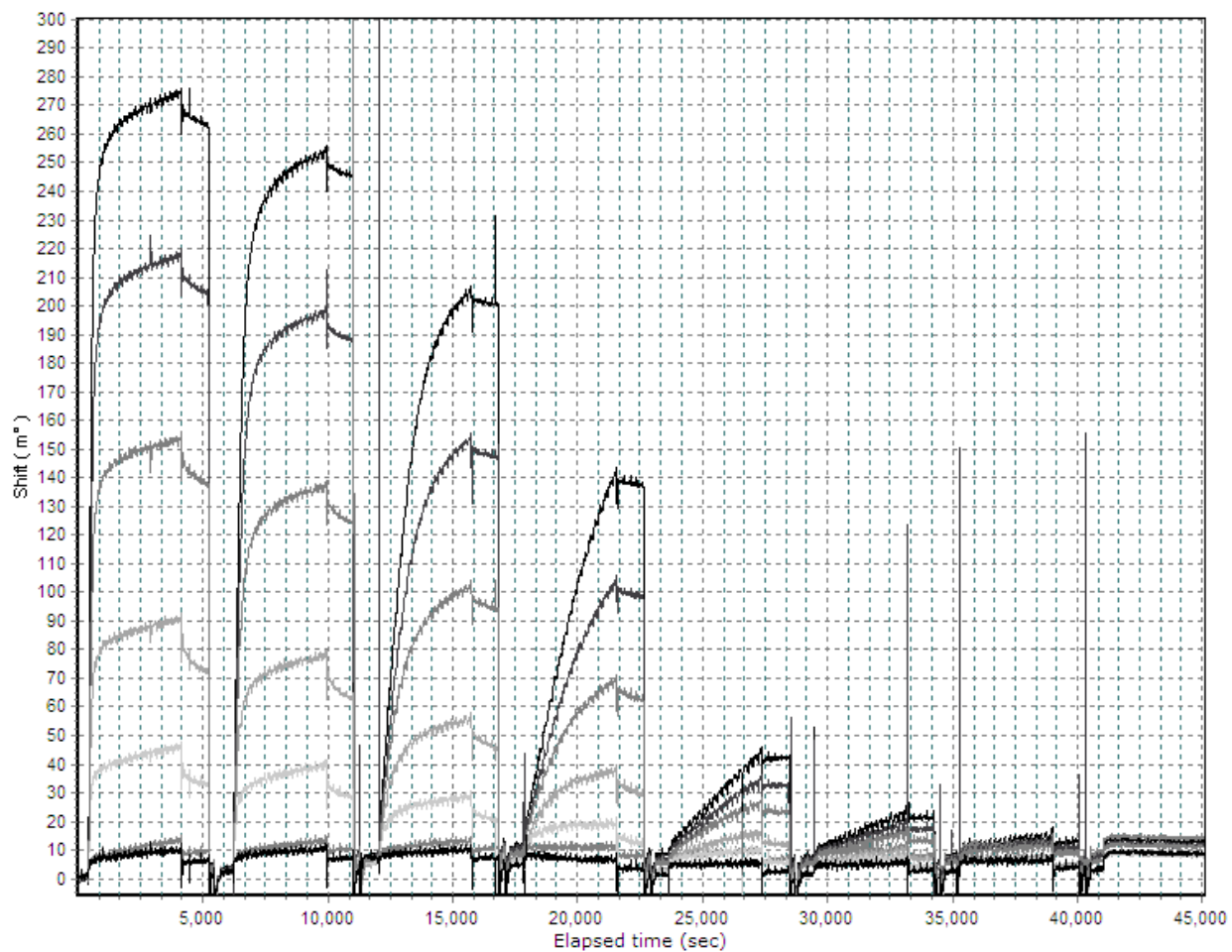

Fig. 4.4. Sensorgram (raw data) of a biotin-antibody interaction experiment with different ligand and analyte concentrations. An injection of the highest analyte mouse anti-biotin mouse IgG (150 kDa) concentration (133 pM) resulted in a gradual decrease in responses for the spots loaded with different ligand biotinylated peptide (2.4 kDa) concentrations obtained by spotting of 417, 208, 104, 52, 26 femtomole and two controls, nonbiotinylated peptide and the background. After this first analysis cycle a lower mouse anti-biotin mouse IgG analyte concentration (53 pM) was injected. In total 8 analysis cycles were carried out in a range of 133, 53, 13, $5.3,1.3,0.53,0.13$ and 0 pM anti-biotin mouse IgG. Each curve shows the transient $2 \mathrm{~m}^{\circ}$ response as a result of the change in flow direction. The few spikes are caused by air bubbles disturbing the SPR signal.

The different curves represent the interaction with the various amounts of biotinylated peptide spotted. The curves varying over time (x-axis) represent the different concentrations of the analyte, anti-biotin IgG. The LOD can be expressed as the smallest 
change in refractive index of the bulk solution or refractive index change in the evanescent field caused by accumulated mass on the sensor surface ${ }^{9}$. If compounds with an increased refractive index replace the background electrolyte buffer solution a linear relation exists between the surface coverage (in \%) and the molecular weight of the adsorbate. However, a low molecular weight compound (e.g. a small peptide) will contribute less to the accumulated mass per surface area compared to a high molecular weight compound (e.g. an immunoglobulin). In literature ${ }^{32}$ it is indicated that the LOD of a certain biomolecular interaction, in terms of mass per square surface area $\left(\mathrm{ng} / \mathrm{mm}^{2}\right)$, depends strongly on the affinity constant of a specific biomolecular interaction. Therefore, the LOD should be expressed in nanogram accumulated mass per square $\mathrm{mm}$.

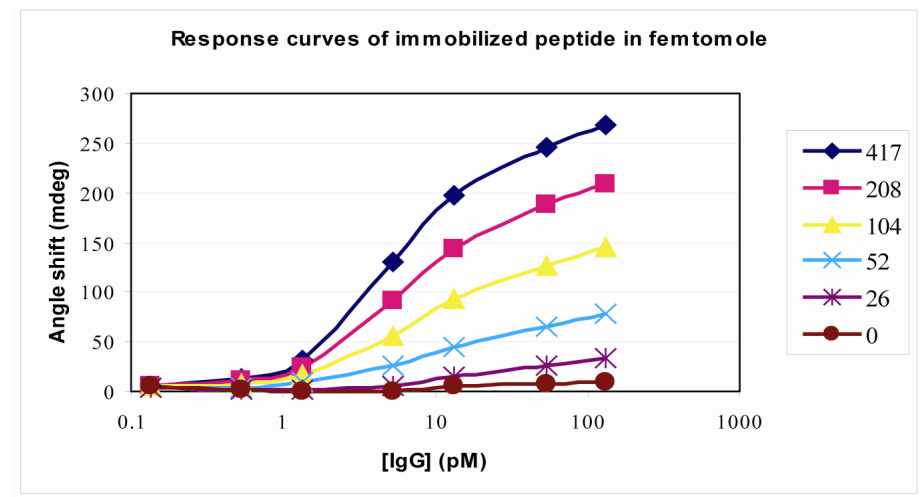

Fig. 4.5. Shows the response curves of the angle shifts in $\mathrm{m}^{\circ}$ (linear scale) which can be derived from Fig. 4.4 from the various peptide concentrations immobilized on the sensor surface and its interactions with various antibiotin IgG concentrations (logarithmic scale).

The molecular mass of the analyte (in this case the anti-biotin IgG $\approx 150 \mathrm{kDa}$ ) gives us an indication how many molecules are needed to detect a significant response compared to the signal to noise ratio. The SPR signal is related to the accumulated mass per surface area (e.g. per spot) the immobilized amount of ligand is depicted in femtomole and the analyte present in solution in picoMolar (pM) (see Fig. 4.5). The amount of analyte bound to the immobilized ligand can be derived from the measured increase in angle shift; $1 \mathrm{~m}^{\circ}=$ $10.8 \mathrm{pg} / \mathrm{mm}^{2}$ as has been determined by refractive index measurements which are dependent on the used wavelength. This results in 1.62 attomole in case of a ROI of 150 
$\mu \mathrm{m} \times 150 \mu \mathrm{m}$, and 64.8 zeptomole or $4.1 \times 104 \mathrm{IgG}$ molecules bound to the minimal ROI of $30 \mu \mathrm{m} \times 30 \mu \mathrm{m}$. Figs. 3 and 4 show the importance of both ligand and analyte concentrations for the achievable maximum angle shift. Immobilization studies showed a maximum relative shift of $17 \mathrm{~m}^{\circ}$ for the peptide and $170 \mathrm{~m}^{\circ}$ for $\operatorname{lgG}$ with respect to the baseline (data not shown). Secondary antibodies and labels like latex particles and colloidal gold ${ }^{33}$ can be used to further increase the mass change thereby improving the LOD. The use of secondary antibodies can even increase the specificity of a reaction; however an additional interaction step has to be included which overrules the favorable label-free one-step measurement. The results in Figs. 7a and 7b show the images taken by SPR and fluorescence microscopy, respectively. The results in Fig. 4.6 represent the signal intensities of the fluorescence image after interaction with $133 \mathrm{pM}$ Alexa Fluor ${ }^{\circledR} 488$ labeled mouse anti-biotin IgG.

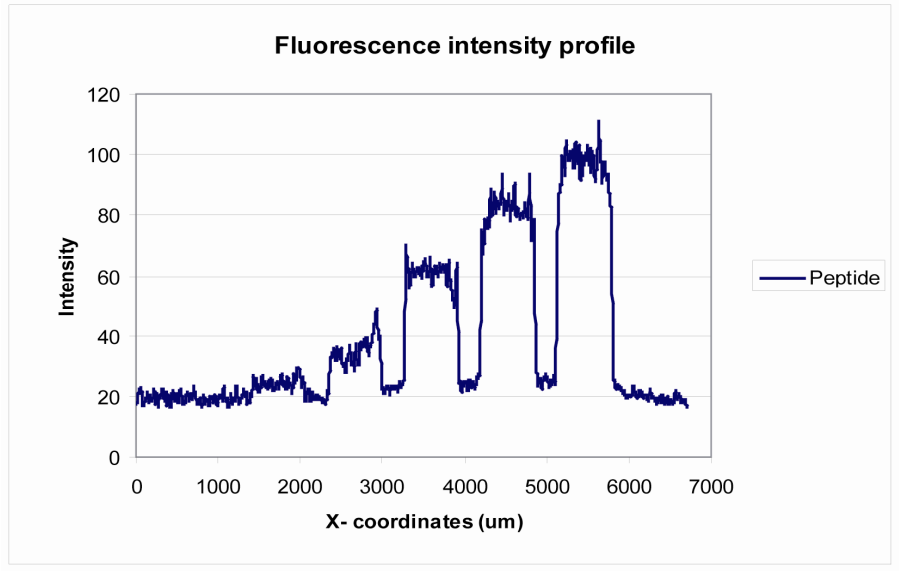

Fig. 4.6. Fluorescence intensity profile of the red line visible in Fig. 4.7b. The ligand concentrations from left to right are: $0,26,52,104,208$ and 417 femtomole immobilized biotinylated peptide and interaction with 133 pM Alexa Fluor 488 labeled mouse anti-biotin IgG.

The interaction at the lowest ligand concentration is barely visible compared to the background. Comparison of the fluorescence results presented in Fig. 4.6 with the results in Fig. 4.4 indicates that SPR is $25-100$ times more sensitive using this experimental setup. This is determined by the significant signal obtained of the lowest ligand concentration, 26 
femtomole, at different analyte concentrations. Quenching of the fluorescence signal by the gold was not observed, which is probably due to the increased distance between the fluorophore and the gold, resulting from the used $200 \mathrm{~nm}$ dextran layer ${ }^{34}$.
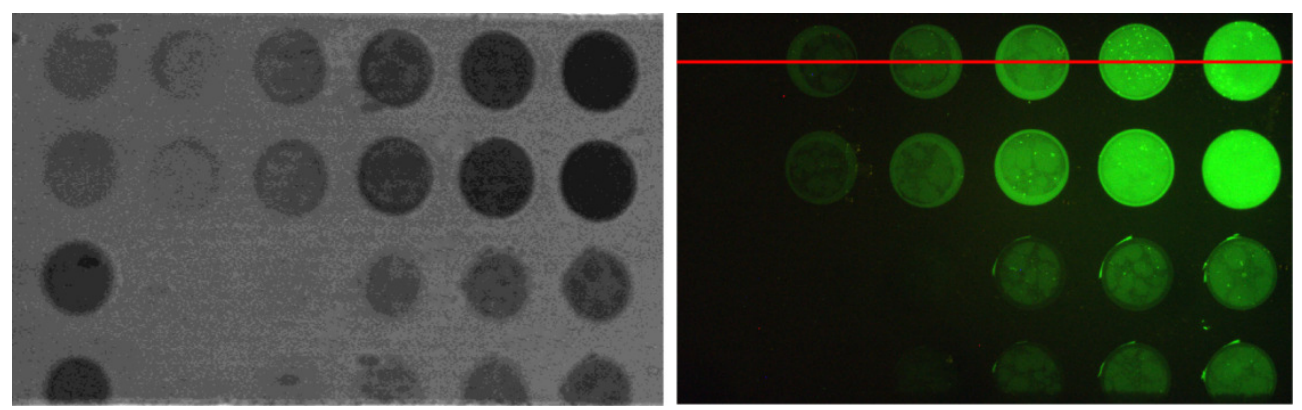

Fig. 4.7a. Imaging SPR image of the microarray of the spotted ligands after interaction with 133pM Alexa Fluor ${ }^{\circledR}$ 488 labeled mouse anti-biotin IgG. Top two rows contain various peptide concentrations spotted in twofold: 417 femtomole of a non-biotinylated peptide as a negative control followed by 26, 52, 104, 208, and 417 femtomole of the biotinylated peptide $(2.4 \mathrm{kDa})$. The bottom two rows contain various IgG concentrations spotted in twofold: 6.67 femtomole of a nonbiotinylated human IgG as a negative control followed by $0.42,0.83,1.67,3.33$ and 6.67 femtomole of biotin-XX goat anti-rabbit IgG $(H+L)(150 \mathrm{kDa})$. Fig. 4.7b. Fluorescence microscopy image of the same microarray of Fig. 4.7a of the spotted ligands after interaction with 133pM Alexa Fluor ${ }^{\circledR} 488$ labeled mouse anti-biotin IgG.

The ability to run the SPR experiments in an automated manner by using liquid handling procedures (LHPs) is an important advantage over many of the previously described systems, and makes it suitable for diagnostic purposes. Due to its flexibility, in terms of sample size and continuous angle-scanning mode, remains the iSPR an interesting tool for research purposes as it can meet individual needs in numerous applications.

\subsection{Conclusion}

The iSPR showed to be a competent and sensitive instrument for investigating many different biomolecular interactions simultaneously because of its large dynamic range. Compared to the fixed angle based SPR imaging systems the angle-scanning mode of the iSPR measures a reliable angle-shift of the SPR-dip, resulting in the 10-fold increased linearity. The angle-scanning SPR imaging facilitates a label-free and real-time imaging of 
microarray interactions within $50 \mathrm{~mm}^{2}$ of the sensor surface, thereby providing the possibility to evaluate the interactions at every spot of the microarray with a real-time image of the surface. The amount of IgG molecules bound to the surface and detected in a label-free manner is $4.1 \times 10^{4}$ molecules or 64.8 zeptomole. Due to the wide range of operation of the instrument both peptide and protein interactions can be measured simultaneously. The obtained results were also successfully verified by means of fluorescence microscopy after completing the SPR imaging experiment.

\subsection{Acknowledgments}

The authors thank The Dutch Technology Foundation (STW) for funding the project TMM 06209 entitled, "Proteomics on a chip for monitoring autoimmune diseases", IBIS Technologies B.V. for their technical support and Verena C. Stimberg for her contribution in the lab.

\subsection{References}

1 Rich, R.L.; Myszka, D.G., Survey of the year 2005 commercial optical biosensor literature. Journal of Molecular Recognition 19, 478-534 (2006).

2 Liedberg, B.; Nylander, C.; Lundstrom, I., Surface plasmon resonance for gas detecting and biosensing. Sensors and Actuators B chemical 4, 299-304 (1983).

3 Kooyman, R.P.H.; Kolkman, H.; Gent, J. van; Greve, J., Surface plasmon resonance immunosensors: sensitivity considerations. Analytica Chimica Acta 213, 35-45 (1988).

4 Lee, H.J.; Wark, A.W.; Corn, R.M., Creating advanced multifunctional biosensors with surface enzymatic transformation. Langmuir 22 (12), 5241-5250 (2006).

5 Kanda, V.; Kariuki, J.K.; Harrison, J.K; McDermott, M.T., Label-free reading of microarray-based immunoassays with surface plasmon resonance imaging. Analytical Chemistry 76, 7257-7262 (2004).

6 Homola, J., Present and future of surface plasmon resonance biosensors. Analytical Bioanalytical Chemistry 377, 528-539 (2003). 
7 Wolfbeis, O.S.; Homola, J., Surface plasmon resonance based sensors. (SpringerVerlag, Berlin, 2006).

8 Wegner, G.J.; Wark, A.W.; Lee, H.J.; Codner, E.; Saeki, T.; Fang, S.; Corn, R.M., Real time SPR imaging measurements for the multiplexed determination of protein adsorption / desorption kinetics and surface enzymatic reactions on peptide microarrays. Analytical Chemistry 76 (5), 5677-5684 (2004).

9 Wolf, L.K.; Fullenkamp, D.E.; Georgiadis, R.M., Single nucleotidepolymorphism genotyping by nanoparticle-enhanced SPR imaging measurements of surface ligation reactions. Journal of the American Chemical Society 127, 17453-17459 (2005).

10 Huang, H.; Chen, Y., Label-free reading of microarray-based proteins with high throughput surface plasmon resonance imaging. Biosensors and Bioelectronics 22, 644-648 (2006).

11 Shumaker-Parry, J.S.; Zareie, M.H.; Aebersold, R.; Campbell, C.T., Microspotting streptavidin and double-stranded DNA arrays on gold for high-throughput studies of protein-DNA interactions by surface plasmon resonance microscopy. Analytical Chemistry 76, 918-929 (2004).

12 Zang, T.; Morgan, H.; Curtis, A.S.G.; Riehle, M., Measuring particle-substrate distance with surface plasmon resosnance microscopy. Journal of Optics A: Pure and Applied Optics 3, 333-337 (2001).

13 Liebermann, T.; Knoll, W., Surface-plasmon field-enhanced fluorescence spectroscopy. Colloids and Surfaces A: Physicochemical and Engineering Aspects $171,115-130$ (2000).

14 Yu, F.; Yao, D.; Knoll, W., Surface plasmon field-enhanced fluorescnece spectroscopy studies of the interaction between an antibody and its surfacecoupled antigen. Analytical Chemistry 75, 2610-2617 (2003).

15 Xiang, J.; Guo, J.; Zhou, F., Scanning electrochemical microscopy combined with surface plasmon resonance: studies of localized film thickness variations and molecular conformation changes. Analytical Chemistry 78, 1418-1424 (2006).

16 Usui-Aoki, K.; Schimada, K.; Nagano, M.; Kawai, M.; Koga, H., A novel approach to protein expression profiling using antibody microarrays combined with surface plasmon resonance technology. Proteomics 5, 2396-2401 (2005). 
17 Hultschig, C.; Kreutzberger, J.; Seitz, H.; Konthur, Z.; Bussow, K.; Lehrach, H., Recent advances of protein microarrays. Current Opinion in Chemical Biology 10, 4-10 (2006).

18 Singh, B.K.; Hillier, A.C., Surface plasmon resonance imaging of biomolecular interactions on a grating-based sensor array. Analytical Chemistry 78, 2009-2018 (2006).

19 Chien, F.-C.; Chen, S.-J., A sensitivity comparison of optical biosensors based on four different surface plasmon resonance modes. Biosensors and Bioelectronics 20, 633-642 (2004).

20 Reather, H., Surface plasmons on smooth and rough surfaces and gratings. (Springer-Verlag, Berlin, 1988).

21 Nelson, B.P.; Frutos, A.G.; Brochman, J.M.; Corn, R.M., Near-infrared surface plasmon resonance measurements of ultrathin films. 1. Angle shift and SPR imaging experiments. Analytical Chemistry 71, 3928-3934 (1999).

22 Nelson, B.P.; Grimsrud, T.E.; Liles, M.R. Goodman, R.M; Corn, R.M., Surface plasmon resonance imaging measurements of DNA and RNA hybridization adsorption onto DNA microarrays. Analytical Chemistry 73, 1-7 (2001).

23 Kretschmann, E.; Reather, H., Radiative decay of non-radiative surface plasmons excited by light. Zeitschrift fur Naturforschung, Teil A. 23A, 2135-2136 (1968).

24 Homola, J.; Yee, S.S.; Gauglitz, G., Surface plasmon resonance sensors: review. Sensors and Actuators B chemical 54, 3-15 (1999).

25 McDonnell, J.M., Surface plasmon resonance: towards an understanding of the mechanisms of biological molecular recognition. Current Opinion in Chemical Biology 5 (5), 572-577 (2001).

26 Katsamba, P.S.; Navratilova, I.; Calderon-Cacia, M.; Fan, L.; Thornton, K.; Zhu, M.; Bos, T. Vanden; Forte, C.; Friend, D.; Laird-Offringa, I.; Tavares, G.; Whatley, J.; Shi, E.; Widom, A.; Lindquist, K. C.; Klakamp, S.; Drake, A.; Bohmann, D.; Roell, M.; Rose, L.; Dorocke, J.; Roth, B.; Luginbühl, B.; Myszka, D. G., Kinetic analysis of a high-affinity antibody/antigen interaction performed by multiple Biacore users. Analytical Biochemistry 352 (2), 208-221 (2006).

27 Säfsten, P.; Klakamp, S.L.; Drake, A.W.; Karlsson, R.; Myszka, D.G., Screening antibody-antigen interactions in parallel using Biacore A100. Analytical Biochemistry 353 (2), 181-190 (2006). 
28 Johnsson, B.; Löfås, S.; Lindquist, G., Immobilization of proteins to a carboxymethyldextran-modified gold surface for biospecific interaction analysis in surface plasmon resonance sensors. Analytical Biochemistry 198 (2), 268-277 (1991).

29 Stigter, E.C.A.; Jong, G.J. de; van Bennekom, W.P., An improved coating for the isolation and quantitation of interferon-[gamma] in spiked plasma using surface plasmon resonance (SPR). Biosensors and Bioelectronics 21 (3), 474-482 (2005).

30 de Heij, B.; Daub, M.; Gutmann, O.; Niekrawietz, R.; Sandmaier, H.; Zengerle, R., Highly parallel dispensing of chemical and biological reagents. Analytical and Bioanalytical Chemistry 378 (1), 119-122 (2004).

31 McIntyre, J.D.E.; Aspnes, D.E., Differential reflection spectroscopy of very thin surface films. Surface Science 24 (2), 417-434 (1971).

32 de Mol, N.J.; , Plomp, E., Fischer, M.J.E., and Ruijtenbeek, R., Kinetic Analysis of the Mass Transport Limited Interaction between the Tyrosine Kinase Ick SH2 Domain and a Phosphorylated Peptide Studied by a New Cuvette-Based Surface Plasmon Resonance Instrument. Analytical Biochemistry 279 (1), 61-70 (2000).

33 Besselink, G.A.J.; Kooyman, R.P.H. van Os, P.J.H.J. Engbers, G.H.M. Schasfoort, R.B.M., Signal amplification on planar and gel-type sensor surfaces in surface plasmon resonance-based detection of prostate-specific antigen. Analytical Biochemistry 333 (1), 165-173 (2004).

34 Cherif, B.; Villiers, C.L.; Pananhos-Baccala, G.; Calemczuk, R.; Marche, P.N.; Livache, T.; Villiers, M.-B., Design and application of a microarray for fluorescence and SPR imaging analysis of peptide-antibody interactions. Journal of Biomedical Nanotechnology 2, 29-35 (2006). 


\title{
5
}

\section{Biomolecular interaction monitoring of autoantibodies by scanning SPR microarray imaging}

\begin{abstract}
This chapter demonstrates a new commercial surface plasmon resonance (SPR) imaging analysis system with a novel SPR dip angle scanning principle allows the measurement, without the need for labeling, of the exact SPR dip angle. With this system hundreds of biomolecular interactions can be monitored on microarrays simultaneously and with great precision. The potency of this system is demonstrated by automatically monitoring the interactions between citrullinated peptides and serum autoantibodies of 50 rheumatoid arthritis (RA) patients and 29 controls in a single step. The smallest antibody concentration that could be measured in this experimental setup was $0.5 \mathrm{pM}$.
\end{abstract}

J. Am. Chem. Soc., 129 (45), $14013-14018,2007$. 


\subsection{Introduction}

Some of the outstanding questions in the fields of biology and medicine remain unsolved as a result of our limited understanding of the function, behavior, and concerted interaction of many significant biomolecules. To obtain information on complex biological systems, protein microarray technology facilitates sensing of multiple biomolecular interactions in parallel. However, labeling is required, which may cause additional problems for studying structure-function relationships of biomolecular interactions. A well-known approach to measure the binding of proteins and other biomolecules to sensor surfaces in a label-free and real-time manner is the surface plasmon resonance (SPR) imaging method, which has been described since $1988^{1} 23^{4}$. SPR is an optical method for measuring the refractive index of material absorbed on a thin metal layer, usually gold. Photons of p-polarized light can interact with the free electrons of the metal layer, inducing a wavelike oscillation of the free electrons and thereby reducing the reflected light intensity. The majority of systems used for SPR imaging represent fixedangle instruments. These instruments are based on a relationship between the change in reflected light intensity and the mass of bound analyte; i.e., a fixed incident light angle is employed and mass changes are estimated from the intensity of the reflected light 4 . However, the applicable range of this linear relationship is limited and the optimal angles differ considerably when ligand or analyte panels having different molecular weights are to be monitored ${ }^{5}$. The use of only one fixed incident light angle is therefore not suitable for the monitoring of multiple biomolecular interactions in parallel on a microarray. Most commercially available instruments for SPR imaging measurements, such as instruments from GWC Technologies, Genoptics, and Kmac, although with different technologies, measure at fixed angles to monitor the interactions.

In systems that use a fixed wavelength the most reliable parameter that directly reflects mass changes on an SPR sensor chip is the SPR dip angle shift ${ }^{67}$. Thus, only when these shifts are monitored for all the spots on a microarray separately, the magnitude and affinity of biomolecular interactions can be reliably compared among all spots of any microarray.

The use of miniaturized and parallelized immunoassays in a microarray format in combination with scanning SPR imaging helps to improve autoimmunity research. Autoimmune diseases are characterized by the presence of high-affinity autoantibodies 
directed against self-proteins, such as rheumatoid factor for rheumatoid arthritis (RA), ${ }^{8}$ Sm for systemic lupus erythemathosus (SLE), ${ }^{9}$ and Ro/SS-A and La/SS-B for Sjögren's syndrome ${ }^{9}$ Although at least some autoantibodies are known to be involved in cell and tissue damage, their mechanistic role in the pathogenesis of the disease is generally not known ${ }^{10}$. Nevertheless, the specificity of autoantibody responses highlights their potential as important tools for improved diagnosis, disease classification, and prognosis. Miniaturized multiplex assays can deliver a fingerprint of a patient's autoantibody repertoire requiring only a limited amount of patient material.

During the past decade various research groups made important contributions to the application of multiplex assays for autoantibody detection. In 2002, Robinson et al. employed protein and peptide ligand arrays, representing candidate autoantigens, to survey autoantibody binding ${ }^{11}$. Arrays of in situ synthesized peptides can also be generated with photolithography to perform antibody characterization ${ }^{12}$. Another approach is to apply "virtual arrays" in a homogeneous assay system with addressable beads ${ }^{13}$. However, in these systems at least one of the interactants must be labeled, which may disrupt the binding sites involved in the interaction, leading to false negatives. In addition, the label itself might interact with the immobilized proteins, leading to false positives ${ }^{14}$. A way to circumvent these problems is to use a labeled secondary binding molecule, which might result in improved assay sensitivity.

In this study scanning SPR microarray imaging (IBIS Technologies, Hengelo, The Netherlands) is used to measure the presence of anti-citrullinated protein antibodies (ACPA) in the sera of RA patients. Recently, it was shown that the so-called citrulline amino acid (which can be generated posttranslationally from arginine) is a critically important moiety of the antigenic determinants targeted by RA-specific autoantibodies ${ }^{15}$. Cyclic citrullinated peptides (CCPS) are widely used as antigenic targets in ELISA-based diagnostic tests for RA. The sensitivity and specificity are $71 \%$ and $99 \%$, respectively ${ }^{16}$. 


\subsection{Results}

\subsubsection{SPR experimental setup}

When a spot meets the optimal SPR conditions, the reflected light at a certain region of interest (ROI) within this spot will reach a minimal value, resulting in a dark spot. Gray values are averaged from the pixels in predefined regions of interest, and in this way the reflected light intensity is determined. The intensity of the reflected light is plotted against the angle of the incident light. SPR measurements carried out in a fixed-angle mode often translate the changes in reflectivity signal to angle shifts of the SPR dip from the linear part of this "reflectivity versus angle plot". In this study the exact SPR dip angle was measured instead of estimated. This was done by fast incident angle scanning within a range of $4^{\circ}$ around the SPR dip angle and imaging the corresponding reflected light via a CCD camera (Fig. 5.1).

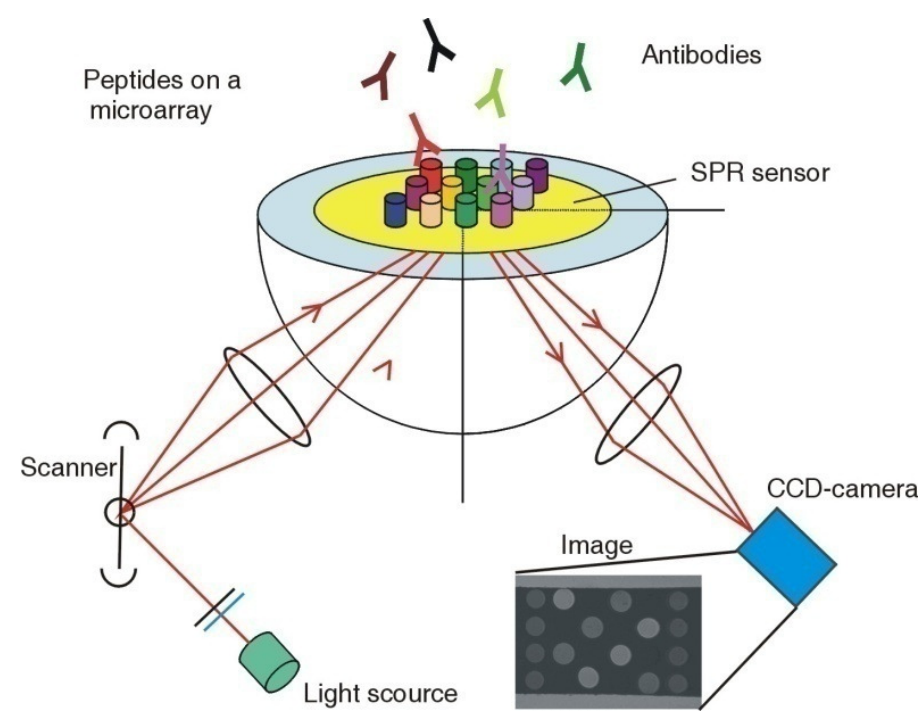

Fig. 5.1 Schematic representation of the optical configuration of the scanning angle SPR imaging instrument. Citrullinated peptides and control peptides are chemically anchored on discrete spots of the microarray. Autoantibodies from diluted rheumatoid arthritis patient serum are flown over the sensor chip. The incident light is being reflected by the angle operated mirror before reaching the hemispheric prism and gold sensor, and the microarray is imaged on a CCD. The exact SPR minimum can be calculated from multiple reflectivity images and monitored for in principle hundreds of regions of interests. 
Scanning is performed stepwise, e.g., an angle scan at steps of $50 \mathrm{~m}^{\circ}$ of incident light, and at each angle an image is taken. By curve fitting the "reflectivity versus angle plots", the angle of the exact SPR dip can be calculated. In contrast to fixed-angle instruments, which measure changes in reflectivity at one defined angle, the sensorgrams generated by the instrument used in this study represent SPR dip angles as a function of time (Fig. 5.2).

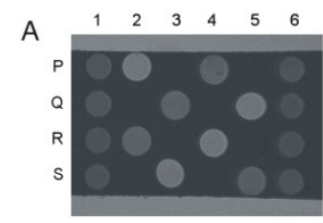

C

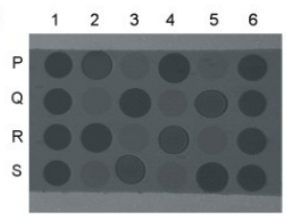

E

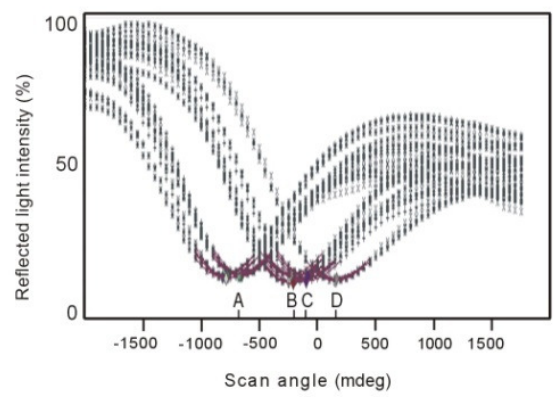

B

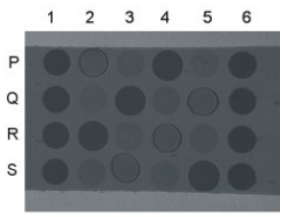

D

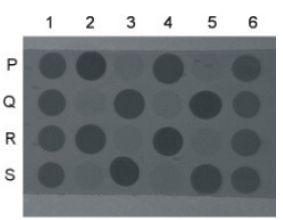

Fig. 5.2 Reflectivity images at four different incident angles and the corresponding reflectivity-versus-angle plot. Spotted array contains in columns 1 and 6, human IgG; spot 4P,3Q,2R,1S, CitA; 2Q,3R,1S,4S, arginine control of CitA, ArgA; spot 2P,5Q,4R,3S, CitB; spot 3P,5P,4Q, 5R, arginine control of CitB, ArgB. Every $50 \mathrm{~m}^{\circ}$ a reflectivity image is taken from which the intensity of the reflected light is measured. This reflected light intensity is plotted against the angle at which the image is taken, which results in a reflectivity-versus-angle plot (E). Incident angle is (A) $-700 \mathrm{~m}^{\circ}$ and the areas surrounding the spots are in resonance; (B) $-200 \mathrm{~m}^{\circ}$, arginine control peptides of both citrullinated peptides are in resonance; (C) $-100 \mathrm{~m}^{\circ}$, citrullinated CitB is in resonance; and (D) $100 \mathrm{~m}^{\circ}$, citrullinated CitA is in resonance. Note from (E) that, if reflectivity values are computed only instead of angle shifts, which is the case in current SPR imaging instruments, the results will be highly inaccurate. 
As a consequence, the $Y$-axis of the sensorgram does not represent an arbitrary reflectivity parameter, but contains the exact values for the SPR angle at maximal resonance. These exact SPR angles can be normalized for easy comparison of all the different sensorgrams of all the spots and are linearly correlated with the refractive index, corresponding to the mass of protein bound to the sensor surface.

\subsubsection{Monitoring the interaction of a peptide array with serum antibodies}

In our proof-of-principle experiments, a 24-spot microarray containing human IgG as well as two different linear citrullinated peptides (CitA and CitB) and the corresponding control peptides (ArgA and ArgB, containing arginine instead of citrulline), were spotted ( $1 \mathrm{~nL}$ per spot) on an $\mathrm{N}$-hydroxysuccinimide preactivated polycarboxylate-coated gold sensor surface using a noncontact spotting instrument ${ }^{17}$.

Images of the reflected light at four different scan angles after incubation of the 24-spot array with serum for 1 hour are shown in Fig. 5.2A-D. The SPR dip of each ROI can be visualized in a reflectivity-versus-angle-plot as shown in Fig. 5.2E. The $0 \mathrm{~m}^{\circ}$ setting is an arbitrary value, which can be set before each individual experiment by manual adjustment to obtain the best scan range. At an incident angle of $-700 \mathrm{~m}^{\circ}$ the background area near the array is in resonance ( $A$ in Fig. 5.2); at $-200 \mathrm{~m}^{\circ}$ the spots coated with the arginine control peptides ArgA and $\operatorname{ArgB}$ are in resonance (B); at $-100 \mathrm{~m}^{\circ}$ the spots coated with citrullinated CitB are in resonance (C); at $100 \mathrm{~m}^{\circ}$ the spots coated with citrullinated CitA are in resonance (D). Fig. 5.2 demonstrates that the intensity profile images differ considerably between ROIs with complexes of varying masses attached to the surface. 


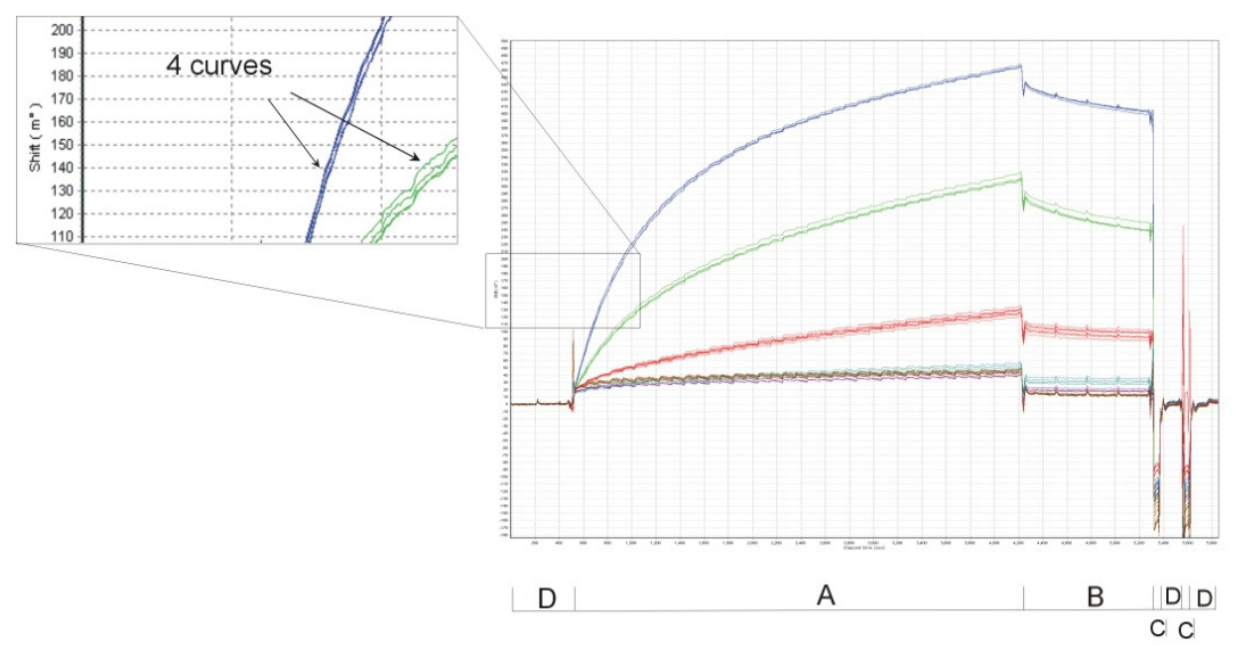

Fig. 5.3 Typical interaction of RA patient antibodies with peptides immobilized on the SPR sensor chip. Sensorgram showing the 24 SPR dip angle shifts as a function of time. Baselines of the 24 regions of interests are zeroed. After the association phase (A), the array was washed with PBS, 0.03\% Tween-20 (B), and regenerated with two sequential steps of $10 \mathrm{mM}$ glycine $\mathrm{HCl}, \mathrm{pH} 1.5$, for $30 \mathrm{~s}(\mathrm{C})$. In between the sequential glycine. $\mathrm{HCl}$ steps the array was rinsed with PBS, 0.03\% Tween-20 (D). Blue (upper) and dark green lines (lowest) are the quadruplicates of CitA and the corresponding arginine control, respectively; light green (second upper) and purple lines are the quadruplicates of citrullinated CitB and the corresponding arginine control, respectively. Red lines are from human IgG $(n=8)$, and the brown lines are from regions close to the array (blank controls) $(n=8)$. The enlarged section shows the four individual curves from the quadruplicates of CitA and CitB.

Fig. 5.3 shows the sensorgrams obtained during incubation of the microarray with an RA serum. ROls in a background region near the array and ROls within the spots of immobilized human IgG and of two arginine-containing peptide controls showed relatively small angle shifts, namely $10,100,18$, and $30 \mathrm{~m}^{\circ}$, respectively. Binding to the two corresponding citrullinated peptides, however, resulted in angle shifts of 250 and $400 \mathrm{~m}^{\circ}$, respectively. The noise, i.e., the baseline difference in resonance angle between the highest value and the lowest value, measured over 100 time points (1000 s) in one individual ROI, was $1.35 \mathrm{~m}^{\circ}$, making angle shifts of $5 \mathrm{~m}^{\circ}$ significant. In Fig. 5.4 the repeatability is shown for various injections of positive RA serum and control normal sheep serum. 


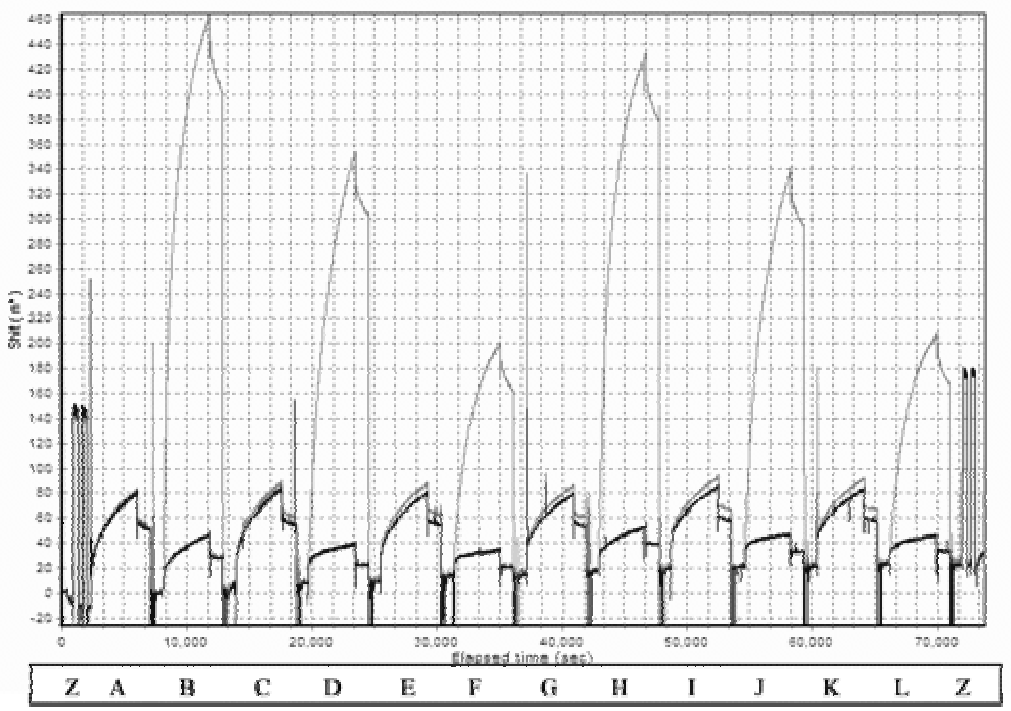

Fig. 5.4 Reproducibility of sequential interactions. Sensorgram (raw data) of a citrulline peptide containing spot and its arginine control spot from the same array as shown in Fig. 5.2. The array was probed two times with three different RA sera: $(B, H)$ serum $1,(D, J)$ serum 2, and $(F, L)$ serum 3. Between each incubation with $R A$ serum the array was incubated with normal sheep serum $(A, C, E, G, I, K)$ to increase the number of regenerations. After every serum (RA serum or NSS) the sensor was regenerated with $10 \mathrm{mM}$ glycine. $\mathrm{HCl}$. Residual serum components after regeneration will form a layer that blocks the aspecific binding sites, which hardly increase after application of more sera. At the beginning and end of the sensorgram a calibration cycle (Z) is performed for sensor calibration purposes by injection two times of a $1 \%$ glycerol solution.

\subsubsection{SPR assay reproducibility}

After placing the spotted sensor chip on top of the hemisphere prism in the flow-cell based instrument, self-defined liquid handling procedures (LHPs) were used to increase the interexperiment reproducibility. Serum incubation, washing, and regeneration were done in an automated manner.

Because none of the peptides contained lysine residues, coupling is expected to occur exclusively via the $\mathrm{N}$-terminal primary amino groups, thereby ensuring oriented, end-on immobilization of the peptides. One set of peptides was synthesized with a C-terminal biotin tag. These peptides were used to investigate possible differences in immobilization efficiency by assessing the SPR angle shift that resulted from incubation of the array with an anti-biotin antibody. The corresponding SPR angle shifts were $211 \pm 10$ and $212 \pm 12 \mathrm{~m}^{\circ}$ 
for the citrullinated peptide and arginine-containing peptide, respectively. From these data it can be concluded that there was no difference in immobilization efficiency between both peptides.

One advantage of multiplexing the monitoring of protein-protein interactions is the ability to test several interactions under the exact same conditions. The intra-array variation in the iSPR system was very low, as is illustrated by the sensorgrams of the quadruplicate interactions in Fig. 5.3. The interaction of the 24-spot array with an RA serum showed an average angle dip shift of $202.5 \mathrm{~m}^{\circ}$ with a standard deviation of $1.3 \mathrm{~m}^{\circ}$.

The sensor chips could be used for up to 50 interaction/regeneration cycles by treatment of the sensor surface with two repetitive incubations with $10 \mathrm{mM}$ glycine. $\mathrm{HCl}, \mathrm{pH} 1.5$, for $30 \mathrm{~s}$ after each serum incubation step. In Fig. 5.4, the repeatability of 12 injections for testing the chip performance is shown. Sequential measurements of patient sera on a single spot showed variations of less than $5 \%$ in binding to the citrullinated peptide, even when six interaction/regeneration cycles were performed between the two sequential measurements.

\subsubsection{Reactivity of patient sera in SPR assay}

The reactivity of sera was quantified by calculating the ratio between the angle shifts observed for the citrullinated peptide $A$ and the corresponding arginine control peptide (hereafter designated $C / R$ ratio) upon binding of serum antibodies. The results for $50 \mathrm{RA}$ sera and 29 control sera (9 SLE patient sera; 10 osteoarthritis (OA) patient sera; 10 normal healthy control sera) are shown in Fig. 5.5. The mean C/R ratio for the RA sera that tested positive in a CCP ELISA (RA CCP+) is 8.6. The C/R ratio for the RA sera that tested negative in the CCP ELISA (RA CCP-), normal controls, SLE patients, and OA patients are 0.96, 1.01, 1.15 , and 1.09 , respectively. Although some RA CCP+ patients showed a $C / R$ around 1 , most of these patients were only weakly positive in the anti-CCP ELISA. Most likely the use of a linear peptide in the SPR assay leads to this slightly reduced sensitivity as the cyclic peptides used in the anti-CCP ELISA are known to be more sensitive. 


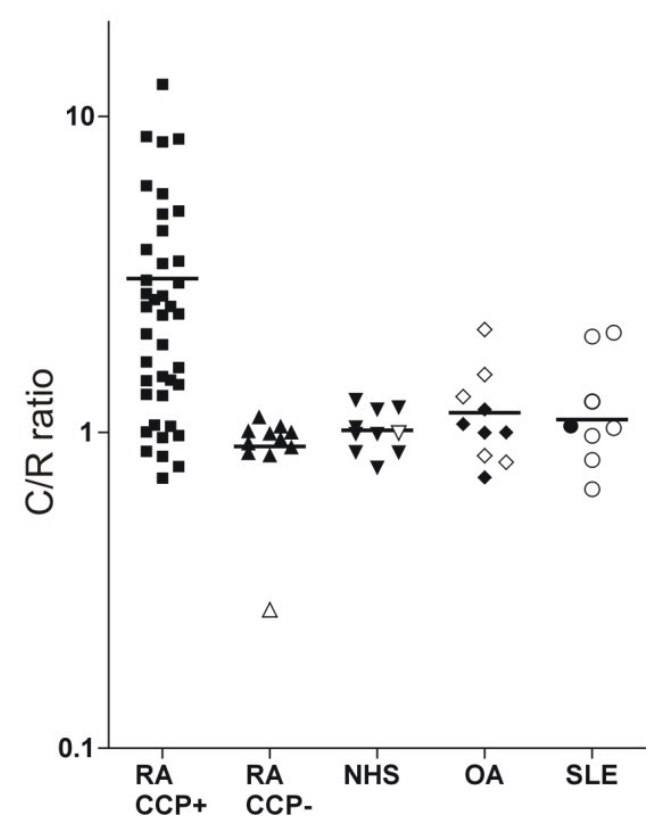

Fig. 5.5 Autoantibody reactivity to a citrullinated peptide monitored by SPR imaging. Fifty RA sera (39 tested positive (RA CCP+) in the CCP ELISA and 11 tested negative (RA CCP-)), 10 normal control sera, 10 OA sera, and 9 SLE sera were tested. Sera for which the citrulline immobilized spots showed an angle shift of less than $10 \mathrm{~m}^{\circ}$ are depicted with open symbols.

\subsection{Discussion}

Surface plasmon microscopy and SPR imaging were first described in $1988^{1234}$. Without exceptions, the systems used for SPR imaging represent fixed-angle instruments; i.e., the mass change is estimated from the intensity of the reflected light ${ }^{4}$. However, the only reliable and underived parameter that directly reflects the concomitant mass change on an SPR-sensor chip is the SPR dip shift ${ }^{6}{ }^{7}$. Thus, only when these shifts are monitored separately for all the spots on a microarray, the magnitude and affinity of biomolecular interactions can be reliably compared among all spots of any microarray. The innovative nature of the approach in this study is the accurateness of SPR dip determination in combination with the unique features of imaging the sensor surface and real-time microarray spot monitoring. In this way the SPR dip of each spot is monitored real-time by 
fast scanning of the angle of incidence and imaging the corresponding reflected light beam via a CCD camera. Currently, SPR dip angles of microarrays of up to 500 ROIs can be determined and this number is limited by the current computing power, since collecting and processing the images including the fast curve-fit calculations for all individual ROls require a huge data transport.

Autoimmune diseases are characterized by the prevalence of autoantibodies recognizing self-proteins. Though many autoantigens have been identified and characterized, to date most of the assays that have been developed to detect autoantibodies to these antigens are ELISA-based, thus allowing only separate analyses for each type of autoantibodies, which is laborious, time-consuming, and not suitable for the development of multiplex systems. Recent observations that the specificity of the detection of anti-CCP antibodies in RA patient sera can be increased by monitoring reactivities with both citrulline- and arginine-containing peptides in parallel emphasize the need for multiplex analysis systems 18. In most of the studies that monitor multianalyte protein-protein interactions, a secondary antibody conjugate was necessary to visualize bound antibodies. Here, we showed that SPR imaging of protein/peptide microarrays provides a method that allows a one-step multianalyte detection of autoantibodies in patient sera, which does not require additional reagents to visualize antibody binding. The ability to measure in a fully automated fashion with liquid handling procedures increases reproducibility, and once an experiment is started it can continue unattended. The ligand-containing sensor chips can be efficiently regenerated and reused, increasing the interassay reproducibility as well.

The smallest antibody concentration that could be measured when a peptide concentration of $1 \mathrm{ng} / \mathrm{nL}$ was used during generation of the array was $0.5 \mathrm{pM}$. Although the sensitivity of detecting RA-specific autoantibodies by the scanning SPR microarray imaging system under the conditions applied in this study is slightly lower than that of the ELISA systems, we expect that further optimization will lead to similar sensitivities. Moreover, for the low-titered sera a signal amplification step, e.g., by using a (goldlabeled) secondary antibody, may raise the sensitivity to levels that allow positive signals for all anti-CCP autoantibody containing sera.

The currently most widely applied target for the detection of anti-citrullinated protein antibodies, cyclic citrullinated peptides, are only recognized by about $70 \%$ of RA patients

${ }^{16}$. Due to the heterogeneity of the anti-citrullinated protein response in RA, ${ }^{15}$ the use of 
additional citrullinated peptides may allow the detection of such antibodies in patients that are not reactive with the peptides used in the CCP2 ELISA. The use of microarrays monitored by SPR imaging will facilitate the simultaneous detection of the various anticitrullinated protein antibodies.

The applicability of this novel scanning SPR technology goes beyond monitoring the presence of autoantibodies in sera of autoimmune patients. Real-time monitoring of the binding allows the user to study the association and dissociation rate constants for determining the affinity constants of the biomolecular interaction. Besides kinetic studies, the accurate measurement of the exact SPR dip angle allows the accurate comparison of each curve, including subtraction of, e.g., common mode effect (i.e., bulk shift jumps). This new scanning SPR technique will be of great use in any field that requires high-detection power and high-throughput analyses.

\subsection{Experimental procedures}

\subsubsection{Serum samples}

The sera were obtained from the Department of Rheumatology, University Hospital Nijmegen. Sera were collected from patients visiting the outpatient clinic who had been diagnosed as having RA according to the revised criteria of the American College of Rheumatology. To further assess specificity, we analyzed a group of serum samples from healthy individuals and groups of sera from patients with osteoarthritis and SLE, obtained from various clinics and hospitals. Sera were stored at $-80^{\circ} \mathrm{C}$ until used.

\subsubsection{Preparation of arrays}

SPR detects changes in refractive index in the hydrogel $(200 \mathrm{~nm})$ which is linked to the gold surface. Due to the small molecular weight of the synthetic peptides used $(\approx 1500$ $\mathrm{Da})$, the contrast of the immobilized array to the background is not high. To visualize the array, human IgG was spotted as well. The peptides and human IgG were spotted using a noncontact spotter ${ }^{17}(1 \mathrm{~nL}$ drops with spotting concentration of $1 \mathrm{ng} / \mathrm{nL}$ in $50 \mathrm{mM} \mathrm{MES}$, pH 5.4) on EDC/NHS-activated Xantec HC $200 \mathrm{~nm}$ sensor chips and placed in a humidity chamber at room temperature for 1 hour. Unreacted active groups were blocked with $1 \mathrm{M}$ 
ethanolamine, $\mathrm{pH}$ 8.0, for 10 min. The sensor chip was rinsed with PBS and placed on a hemisphere with index-matching oil and inserted in the SPR microarray imaging instrument.

\subsubsection{SPR microarray interaction studies}

Incubation, washing, and regeneration were performed in an automated way using liquid handling procedures (LHPS) in the instrument for biomolecular interaction sensing (IBISiSPR, IBIS Technologies BV, Hengelo, The Netherlands). A serum sample plug of $400 \mu \mathrm{I}$ (diluted 1:50 in PBS, 0.03\% Tween-20) was guided backward and forward over the array in a flow-cell with a speed of $1 \mu \mathrm{l} / \mathrm{s}$. The serum sample plug was surrounded by two air plugs to prevent the diffusion of serum components into the buffer. Between all steps the flowcell was rinsed with PBS, $0.03 \%$ Tween-20. The array was regenerated by injection of 400 $\mu \mathrm{l}$ of $10 \mathrm{mM}$ glycine. $\mathrm{HCl}, \mathrm{pH} 1.5$, twice for $30 \mathrm{~s}$. Two incubation/regeneration cycles were completed before applying the sera in order to block aspecific binding sites and create an optimal reactive sensor surface. Analysis of the data was done using the supplied software.

\subsubsection{Peptide ELISA}

Anti-CCP2 ELISA was performed by IMMUNOSCAN RA (Euro-Diagnostica, Arnhem, The Netherlands), in accordance with the manufacturer's instructions with the recommended $25 \mathrm{U} / \mathrm{ml}$ cutoff.

\subsection{Acknowledgment}

We thank Dr. Jan Wouter Drijfhout (Leiden University Medical Centre, The Netherlands) for providing the synthetic peptides used in this study. This research is supported by the Dutch Technology Foundation STW, applied science division of NWO, and the Technology Program of the Ministry of Economic Affairs. 


\subsection{References}

1 Rothenhauserler, B.; Knoll, W., Surface plasmon microscopy. Nature 332, 615-617 (1988). Jordan, C.E.; Frutos, A.G.; Thiel, A.J.; Corn, R.M., Surface Plasmon Resonance Imaging Measurements of DNA Hybridization Adsorption and Streptavidin/DNA Multilayer Formation at Chemically Modified Gold Surfaces. Analalytical Chemistry 69 (24), 4939-4947 (1997).

3 Thiel, A.J.; Frutos, A.G.; Jordan, C.E.; Corn, R.M.; Smith, L.M., In Situ Surface Plasmon Resonance Imaging Detection of DNA Hybridization to Oligonucleotide Arrays on Gold Surfaces. Analytical Chemistry 69 (24), 49484956 (1997).

Nelson, B.P.; Frutos, A.G.; Brochman, J.M.; Corn, R.M., Near-infrared surface plasmon resonance measurements of ultrathin films. 1 . Angle shift and SPR imaging experiments. Analytical Chemistry 71, 3928-3934 (1999). Nelson, B.P.; Grimsrud, T.E.; Liles, M.R. Goodman, R.M; Corn, R.M., Surface plasmon resonance imaging measurements of DNA and RNA hybridization adsorption onto DNA microarrays. Analytical Chemistry 73, 1-7 (2001). Liedberg, B.; Nylander, C.; Lundstrom, I., Surface plasmon resonance for gas detecting and biosensing. Sensors and Actuators B chemical 4, 299-304 (1983).

7 Liedberg, B.; Nylander, C.; Lundstrom, I., Biosensing with surface plasmon resonance -- how it all started. Biosensors and Bioelectronics 10 (8), i-ix (1995). Publishers, Dordrecht, NL, 1994).

Routsias, J.G.; Tzioufas, A.G.; Moutsopoulos, H.M., The clinical value of intracellular autoantigens B-cell epitopes in systemic rheumatic diseases. Clinica Chimica Acta 340 (1-2), 1-25 (2004).

10 F.A. van Gaalen, S.P. Linn-Rasker W.J. van Venrooij B.A. de Jong F.C. Breedveld C.L. Verweij R.E.M. Toes T.W.J. Huizinga, Autoantibodies to cyclic citrullinated peptides predict progression to rheumatoid arthritis in patients with undifferentiated arthritis: A prospective cohort study. Arthritis \& Rheumatism 50 (3), 709-715 (2004). 
11 Robinson, W.H.; DiGennaro, C.; Hueber, W.; Haab, B.B.; Kamachi, M.; Dean, E.J.; Fournel, S.; Fong, D.; Genovese, M.C.; Neuman de Vegvar, H.E.; Skriner, K.; Hirschberg, D.L; Morris, R.I.; Muller, S.; Pruijn, G.J.; Venrooij, W.J.van; Smolen, J.S.; Brown, P.O.; Steinman, L.; Utz, P.J., Autoantigen microarrays for multiplex characterization of autoantibody responses. Nature Medicine 8 (3), 295-301 (2002).

12 Fodor, S.P.; Read, J.L.; Pirrung, M.C.; Stryer, L.; Lu, A.T.; Solas, D., Lightdirected, spatially addressable parallel chemical synthesis. Science $251,767-$ 773 (1991).

13 Fulton, R.J.; McDade, R.L.; Smith, P.L.; Kienker, L.J.; Kettman, J.R., Jr., Advanced multiplexed analysis with the FlowMetrixTM system. Clinical Chemistry 43 (9), 1749-1756 (1997).

14 Cooper, M., Label-free screening of bio-molecular interactions. Analytical and Bioanalytical Chemistry 377 (5), 834-842 (2003).

15 Schellekens, G.A.; Jon, B.A. de; Hoogen, F.H. an den; Putte, L.B. van de; Venrooij, W.J. van, Citrulline is an esential constituent of antigenic determinants recognized by rheumatoid arthritis-specific autoantibodies. The Journal of Clinical Investigation 101 (1), 273-281 (1998). van Venrooij, W. J.; Zendman, A.t J. W.; Pruijn, G.J.M., Autoantibodies to citrullinated antigens in (early) rheumatoid arthritis. Autoimmunity Reviews 6 (1), 37-41 (2006).

17 Gutmann, O.; Niekrawietz, R.; Kuehlewein, R.; Steinert, C. P.; Reinbold, S.; Heij, B. de; Daub, M.; Zengerle, R., Non-contact production of oligonucleotide microarrays using the highly integrated TopSpot nanoliter dispenser. The Analyst 129 (9), 835-840 (2004).

18 Vannini, A.; Cheung, K.; Fusconi, M.; Stammen-Vogelzangs, J.; Drenth, J.P.H.; Dall'Aglio, A.C.; Bianchi, F.B.; Bakker-Jonges, L.E.; van Venrooij, W.J.; Pruijn, G.J.M.; Zendman, A.J.W., Anti-cyclic citrullinated peptide positivity in nonrheumatoid arthritis disease samples: citrulline-dependent or not? Annals of the Rheumatic Disease 66, 511-516 (2007). 



\section{Ratio-diluted ligand density assays for single analyte kinetic analysis}

This chapter describes a new assay protocol based on injecting a single analyte concentration, to a microarray of immobilized ligands with various densities. This new approach is different when compared to the conventional approach where multiple analyte injections of varying analyte concentrations are introduced to ligands of a single density. The new method uses controlled dilution of ligands with background molecules which facilitates immobilization of a precise ligand density on the surface, which is important for direct kinetic analysis. This alternative to the conventional multi-analyte overlay plot for calculating the rate-and affinity constants of a biomolecular interaction, has the advantage of requiring only a single analyte injection, fewer surface regeneration steps, and a reduction of the overall assay time. As a model system, various biotin specific interactions have been tested by means of SPR imaging for their affinity toward surface immobilized biotinylated peptides.

Manuscript in preparation. 


\subsection{Introduction}

Surface plasmon resonance (SPR) biosensors have been used since the 1980s to study biomolecular interactions ${ }^{1}$, and the powerful method of SPR imaging was introduced shortly after ${ }^{2}$. At the moment there are several commercial SPR imaging instruments available, e.g. Biacore Flexchip (GE Healthcare, Uppsala, Sweden), ProteOn ${ }^{\mathrm{TM}}$ XPR36 (BioRad Laboratories, Hercules, USA), SPRimager ${ }^{\circledR}$ II ARRAY system (GWC Technologies, Madison, USA), SPRi-Plex ${ }^{\mathrm{TM}}$ (GenOptics Bio Interactions, Orsay, France) and IBIS-iSPR (IBIS Technologies B.V., Hengelo, The Netherlands) ${ }^{3}$. Other SPR configurations have been reported for multi-analyte biosensing ${ }^{4}$.

SPR is a well established label-free and real-time measurement method that can be used to study the kinetics of biomolecular interactions at the sensor surface ${ }^{6}{ }^{7}$. This is important because conventional methods, e.g. fluorescent microarrays indicate ligandanalyte binding, but temporal or kinetic information is typically not available. The kinetic information provides an additional level of ligand-analyte discrimination and it is anticipated to play an important role in molecular binding assays for many applications ${ }^{89}$.

The conventional method of kinetic analysis is to collect SPR responses using various analyte concentrations exposed to a sensor surface with a single ligand density ${ }^{10}$. During this process the ligand sites at the surface are used multiple times and must be regenerated prior to each analysis cycle containing a different analyte concentration, as shown in Fig. 6.1. The surface regeneration step has several disadvantages such as varying ligand-analyte binding resulting in non-homogeneous regeneration, and ligand damage due to harsh chemistry or multiple regeneration steps. One way to avoid regeneration is by applying kinetic titration ${ }^{11} 12$, where multiple analyte concentrations are injected sequentially from a low to high concentration without regenerating the surface. The algorithms used to analyze the data are compared to the conventional experimental procedures, and give similar results. Another way to avoid regeneration of the surface, is to parallelize the analyte flow perpendicular over the ligand surface as done by the ProteOn XPR36 array biosensor ${ }^{13}$, creating a checkerboard of $6 \times 6$ interactions on the surface. 


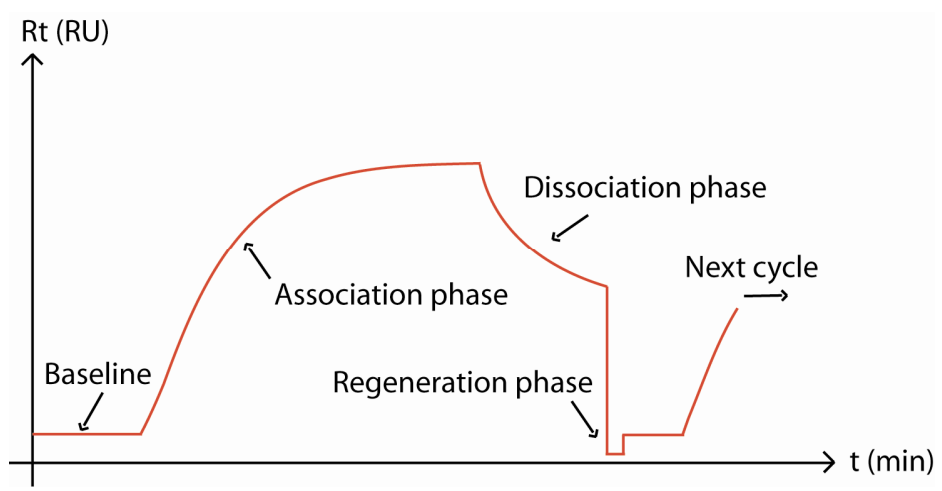

Fig. 6.1. The analysis cycle contains a baseline, association, dissociation and regeneration phases. The association rate $k_{a}$, dissociation rate $k_{d}$ and affinity constant $K_{D}\left(K_{D}=k_{d} / k_{a}\right)$ can be extracted from the data using properly chosen response models and is discussed in the next section. $R_{t}$ is the SPR response, where $R U$ is the response unit, resonance unit or refractive index unit e.g. angle in $\mathrm{m}^{\circ}$ or $\%$ reflectivity, measured at time $\mathrm{t}(\mathrm{sec})$.

In the case of the Octet from ForteBio (Menlo Park, CA, USA) ${ }^{14}$, a disposable interferometry system, the optical fiber biosensors (tips) are dipped into the analyte solution and discarded after the readout. All of the above approaches require the use of various analyte concentrations, in a sequential, parallel or disposable manner, to calculate the kinetics constants.

Here we propose to parallelize analyte interactions on spots with varying ligand densities in a microarray format, instead of varying the analyte concentrations in order to totally avoid or decrease the regeneration steps. Very short immobilization times can contribute to more controlled ligand densities obtained at the sensor surface however the exact amounts remain difficult to control, especially in a microarray format ${ }^{15}{ }^{16}$. The dilution with a background molecule results in a constant amount of binding molecules in the spotting solution and, therefore, a more controlled dilution of the active ligand at the sensor surface ${ }^{17}$. The dilution should preferably be done with a molecule having similar coupling kinetics. The concept of ratio-diluted surface immobilization is shown schematically in Fig. 6.2, where the total amount of ligand on the surface is always kept $100 \%$ (saturated) in the case of the ratio-dilution, with varying active biomolecule densities. The amount of molecules on the surface of the buffer-dilution is ideally reduced according to the dilution factor, however the immobilization rate, the depletion factor of 
ligand during immobilization and surface capacity influence the actual amount of molecules at the surface.

\begin{tabular}{|c|c|c|c|c|c|}
\hline \multicolumn{2}{|c|}{ Analyte dilution } & \multicolumn{2}{|c|}{ Ligand dilution: buffer } & \multicolumn{2}{|c|}{ Ligand dilution: ratio } \\
\hline$\therefore \therefore$ & YYYYYYYY & $\therefore$ & YYYYYYYY & $\therefore$ & YYYYYYYY \\
\hline$\therefore$ & YYYYYYYY & $\therefore$ & Y Y Y Y & $\therefore$ & $Y \perp Y \perp Y \perp Y \perp$ \\
\hline$\therefore$ & YYYYYYYY & $\therefore$ & $Y$ & $\therefore$ & $\Upsilon \perp|\perp| \perp \mid$ \\
\hline & $=$ Analyte conce & & YYY & $=\mathrm{Lig}$ & density \\
\hline
\end{tabular}

Fig. 6.2. Schematic representation of the surface ligand density, Ratio-dilution contains a mixture of biotinylated peptides and non-biotinylated peptides functioning as background molecules. In the buffer-dilution the biotinylated peptides are diluted with immobilization buffer, in this case morpholinoethanesulfonic acid monohydrate, MES-buffer.

Due to the reduction of analyte cycles needed to perform kinetic analysis, both the total analyte sample volume and measurement time are significantly reduced. Instead of making analyte dilutions, ligand dilutions are immobilized on the surface. The applicability for higher-throughput kinetic analyses of microarrays without the need for regeneration, are numerous $\begin{array}{llll}11 & 18 & 19 & 9\end{array}$. Other advantages are savings in time and costs if precious biomolecules are used. The advantages and disadvantages of the different dilution methods used are listed below in Table 6.1.

\begin{tabular}{l|ccc} 
Attribute & Analyte dilution & Ligand dilution buffer & Ligand dilution ratio \\
\hline Surface regeneration & - & $+/-$ & + \\
Analyte & - & $+/-$ & + \\
Time & - & - & + \\
Controlled ligand density & NA & - & + \\
Visibility of microarray & NA & & +
\end{tabular}

Table 6.1. advantages and disadvantages of the various dilution methods. An advantage is indicated with + , disadvantage with -, when the effect is not completely clear with +/-, and if not applicable with NA. 
The applicability of commonly used kinetic analysis software is demonstrated for ratiodiluted ligands, and is compared to the standard analyte overlay plot procedure.

\subsection{Materials and methods}

\subsubsection{Reagents}

1-Ethyl-3-(3-dimethylaminopropyl)carbodiimide (EDC), N-hydroxysuccinimide (NHS), ethanolamine, phosphate buffered saline (PBS), glycine. $\mathrm{HCl}$ and monoclonal anti-biotin FITC conjugated [Mouse] (\# F4024) were purchased from Sigma-Aldrich Chemie GmbH (Steinheim, Germany). Glycerol and Tween 20 were purchased from Fluka Chemie GmbH (Buchs, Switzerland). Fab fragment of affinity purified anti-biotin [Goat] (\# 800-101-098), $\mathrm{F}(\mathrm{ab})_{2}$ fragment of affinity purified anti-biotin [Goat] (\# 700-101-098) and affinity purified anti-biotin [Goat] (\# 600-101-098) were purchased from Rockland Inc. (Gilbertville, USA). Monoclonal anti-biotin Alexa Fluor ${ }^{\circledR} 488$ conjugate [Mouse] (\# A31801) was purchased from Molecular Probes Europe BV (Leiden, The Netherlands). Morpholinoethanesulfonic acid (MES) monohydrate and acetic acid were purchased from Merck KGaA (Darmstadt, Germany). Refractive index matching liquid $n=1.518 \pm 0.0002$ from R.P. Cargille Laboratories Inc. (Cedar Grove, USA). VLSI Selectipur ${ }^{\circledR}$ absolute ethanol was purchased from BASF Electronic Materials GmbH (Ludwigshafen, Germany), Assistend ${ }^{\circledR}$ optical paper was purchased from Omnilabo International B.V. (Breda, The Netherlands) and RBS Neutral was purchased from Chemical Products s.a. (Brussels, Belgium). All buffer solutions were prepared with Milli-Q water $(\mathrm{pH}=5.52$, conductivity $=0.65 \mu \mathrm{S} / \mathrm{cm})$.

\subsubsection{Microarray fabrication}

A $100 \mathrm{~nm}$ thick polycarboxylated functionalized hydrogel (G-COOH-sp) sensor disc from IBIS Technologies B.V. (Hengelo, The Netherlands), was activated by shaking for $20 \mathrm{~min}$ at room temperature with 0.4 M EDC/0.1 M NHS in MES buffer $(50 \mathrm{mM}, \mathrm{pH} 5.4)^{20}{ }^{21}$. The activated surface was rinsed with acetic acid $(50 \mathrm{mM}, \mathrm{pH} 3.0)$ and dried under vacuum for 20 min. A TopSpot device in combination with a 24-channel printhead, from BioFluidix $\mathrm{GmbH}$ (Freiburg, Germany) ${ }^{22}$, was used to spot the biotinylated peptides in a non-contact manner on the sensor surface. The dilution method is illustrated in Fig.6.3, and the layout 
of the array is described in the caption of Fig. 6.6. Between various spotting runs the printhead was cleaned in a cleaning station in a ultrasonic bath and flushed with a $5 \%$ RBS cleaning solution and Milli-Q water to prevent cross-contamination. The 24 spot microarray, $4 \times 6$, on top of the sensor disc consists of spots of approximately $300 \mu \mathrm{m}$ in diameter and with a pitch of $500 \mu \mathrm{m}$. The sensor was incubated in a humidity chamber at room temperature, to prevent drying of the spotted $1 \mathrm{nl}$ droplets during the 1 hour immobilization process. After immobilization, the remaining active groups of the sensor surface were quenched with ethanolamine $(1 \mathrm{M}, \mathrm{pH} 8.0)$ for $10 \mathrm{~min}$ at room temperature. The sensor was kept in PBST (PBS/0.03\% Tween 20) pH 7.4, before the measurement to prevent drying of the microarray.

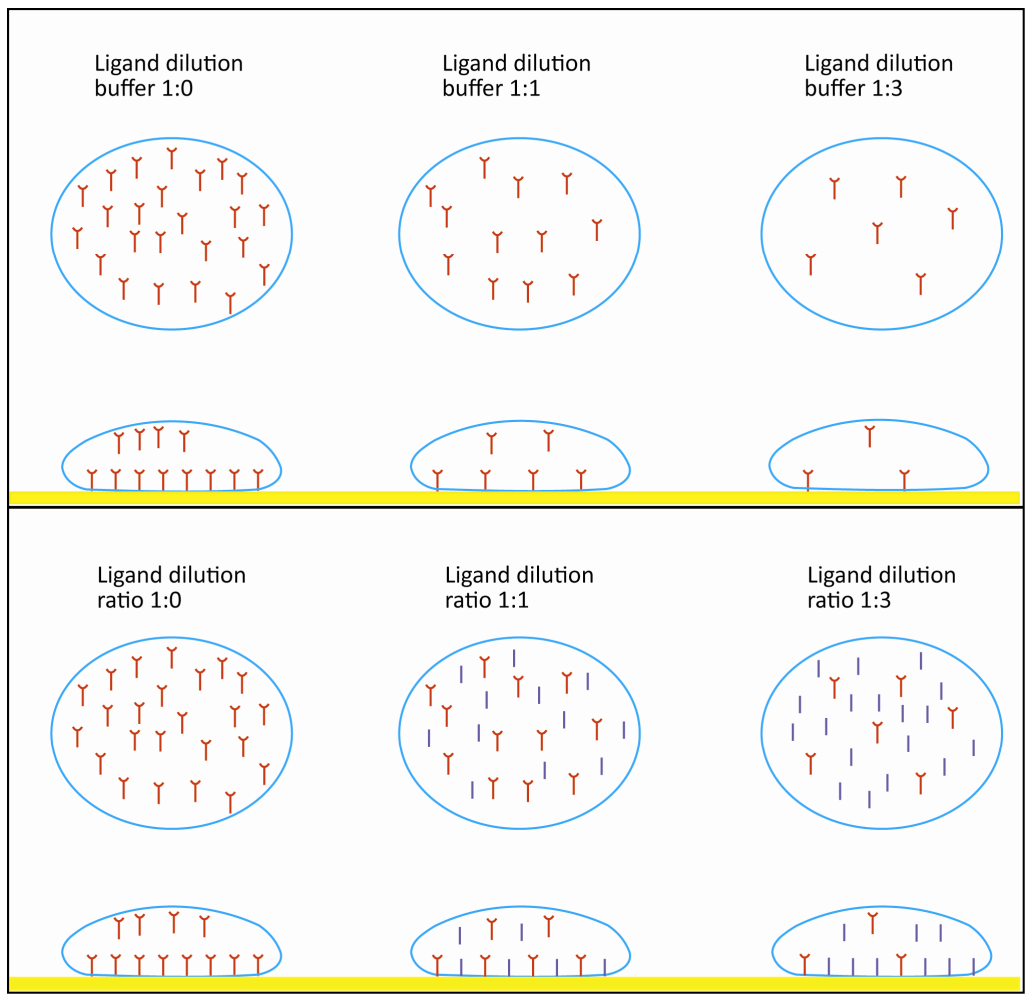

Fig. 6.3. Represents the spotted droplets containing the various ligand concentrations and the resulting ligand densities at the sensor surface for the buffer- and ratio-dilution methods. In the ratio-dilution, the concentration of biomolecules remains constant. 


\subsubsection{SPR imaging setup}

The IBIS-iSPR system, from IBIS Technologies B.V. (Hengelo, The Netherlands) was used for this study. In the SPR imaging system, the light is reflected on an angle-controlled mirror (range $8^{\circ}$ ) before passing through a hemispherical prism to enable fast scanning of the angle shift of the SPR-dip of the individual regions of interest (ROIs) ${ }^{23}{ }^{24}$. The microarray containing sensor disc is placed carefully on the clean prism using a droplet of refractive index matching liquid ( $n=1.518$ ) to avoid entrapment of air-bubbles. A $3 \mu \mathrm{l}$ flow-cell, with chamber dimensions of $10 \mathrm{~mm} \times 3 \mathrm{~mm} \times 0.1 \mathrm{~mm}(\mathrm{I} \times \mathrm{w} \times \mathrm{h})$, is positioned over the 24 spot microarray. The $1 \mathrm{ml}$ syringe is connected to the flow-cell and the flow-cell is connected to the autosampler needle according to Fig. 3.2. The flow-cell is temperature controlled by Peltier elements. Liquid handling procedures (LHPs / scripts) are written in the iSPR software (version 4) to navigate the liquid-handler and thereby facilitating a fully automated performance of multiple analysis cycles of an experiment.

\subsubsection{Analysis cycle}

The sensor containing the peptide microarray was mounted in the SPR imaging system and equilibrated for $5 \mathrm{~min}$ using $1 \mathrm{ml} \mathrm{PBST}$ in the flow-cell at a mixing volume of $20 \mu \mathrm{l}$ and -flow rate of $30 \mu \mathrm{l} / \mathrm{s}$ at $25^{\circ} \mathrm{C}$. After defining the ROls of $300 \mu \mathrm{m} \times 300 \mu \mathrm{m}$, the SPR-dip was determined automatically by the IBIS software. After a regeneration step to remove the unbound ligand, a zero-line was made in PBST. Signals were determined according to the difference in angle-shift between the PBST wash-step after interaction and the PBST baseline before the interaction. The mixing flow rate is kept constant at $30 \mu \mathrm{l} / \mathrm{s}$ throughout the various parts of the analysis cycle. The analysis cycle contains a regeneration step of using $150 \mu \mathrm{l} 10 \mathrm{mM}$ glycine $\cdot \mathrm{HCl}, \mathrm{pH} 1.5(50 \mu \mathrm{l}$ mixing volume), a 120 sec baseline in $150 \mu \mathrm{l}$ PBST (50 $\mu \mathrm{l}$ mixing volume), a $600 \mathrm{sec}$ association phase of $100 \mu \mathrm{l}$ biotin-binding analyte in PBST ( $20 \mu \mathrm{l}$ mixing volume), and a $300 \mathrm{sec}$ dissociation phase in $150 \mu \mathrm{l}$ PBST ( $50 \mu \mathrm{l}$ mixing volume). Association times of $900 \mathrm{sec}$ and dissociation times of $600 \mathrm{sec}$ have also been used.

\subsubsection{Kinetic analysis}

The standard way to determine the kinetic behavior of a biomolecular interaction is by making an analyte overlay plot to calculate the kinetic constants; association rate $k_{a}$, 
dissociation rate $k_{d}$, affinity constant $K_{D}$ and maximal response $R_{\max }$. Currently the majority of kinetic data analysis is initially performed using a 1:1 interaction model ${ }^{25263}$,

$$
A+B \underset{k_{d}}{\stackrel{k_{a}}{\rightarrow}} A B
$$

where $A$ is the analyte in solution, $B$ is the immobilized ligand on the surface, $A B$ is the complex formed on the surface, $k_{a}$ and $k_{d}$ are the association and dissociation rate constants, respectively. At equilibrium the association rate is equal to the dissociation rate,

$$
k_{a}[A][B]=k_{d}[A B]
$$

the equilibrium constants $\mathrm{K}_{\mathrm{A}}$ and $\mathrm{K}_{\mathrm{D}}$ can be expressed by the rate constants by rearranging equation (2) to,

$$
K_{A}=\frac{[A B]}{[A][B]}=\frac{k_{a}}{k_{d}}
$$

and

$$
K_{D}=\frac{[A][B]}{[A B]}=\frac{k_{d}}{k_{a}}
$$

During complex formation the association rate is higher than the dissociation rate according to the differential equation,

$$
\frac{d[A B]}{d t}=k_{a}[A][B]-k_{d}[A B]>0
$$

The complex formed at the surface $[A B]$ is linearly correlated to the response $R_{t}$. So the measured response $R_{t}$ (Fig. 6.1), scales with the complex formation $[A B]$ and thus the increased mass on the sensor surface ${ }^{27}$. If the ligand surface concentration is small compared to the analyte in solution then the association rate controlling and the ligandanalyte reaction can be described by pseudo-first-order kinetics with the rate equation ${ }^{28}$. 


$$
\frac{d R_{t}}{d t}=k_{a}[A]\left(R_{\max }-R_{t}\right)-k_{d} R_{t}
$$

Where $R_{t}$ is the measured response from the SPR system and $R_{\max }$ is the response associated with maximum amount of analyte that can bind to the immobilized ligand. Integrating the first order differential equation (6) and using the initial boundary conditions $R_{t}(t=0)=0$ results in the solution,

$$
R_{t}=\frac{k_{a}[A] R_{\max }}{k_{a}[A]+k_{d}}\left(1-e^{-\left(k_{a}[A]+k_{d}\right) t}\right)
$$

where $\mathrm{K}_{\mathrm{D}}$ is the equilibrium constant $K_{D}=\frac{k_{d}}{k_{a}}$. The equilibrium response i.e. $\mathrm{t} \rightarrow \infty$

$$
R_{t}(e q)=\frac{k_{a}[A] R_{\max }}{k_{a}[A]+k_{d}}
$$

Since equations (7) and (8) require the dissociation rate constant in order to calculate $k_{a}$, then dissociation of bound analyte can be induced in a continuous buffer flow, and therefore, the rate equation can be reduced to,

$$
\frac{d R_{t}}{d t}=-k_{d} R_{t}
$$

Integrating equation (9) with initial boundary conditions $R_{t}(t=0)=R_{0}$ results in

$$
R_{t}=R_{0} e^{-k_{d} t}
$$

where $R_{0}$ is starting response of the dissociation phase. From equation (10), the dissociation rate can be extracted, using a nonlinear fitting method, from the measured response. Once $k_{d}$ is determined, then $k_{a}$ can be extracted from the measured response with known $[A]$ and $R_{\max }$. $R_{\max }$ can be determined experimentally by a saturating 
concentration of $[\mathrm{A}]^{29}$, or an assumption can be made by adding an additional fitting parameter $\mathrm{R}_{\mathrm{i}}{ }^{28}$,

$$
R_{t}=\frac{k_{a}[A] R_{\max }}{k_{a}[A]+k_{d}}\left(1-e^{-\left(k_{a}[A]+k_{d}\right) t}\right)+R_{i}
$$

with $R_{i}$ being much larger compared to $R_{\max }$. Alternatively, $R_{\max }$ can be extracted from the data with the model in equation (9).

Due to the complexities, many of the SPR users try to fit their data according to the pseudo-first-order kinetics, which has been implemented in the software package, Scrubber (version 2.0, BioLogic, Campbell, Australia) ${ }^{30} 31$, meaning the reaction depends on the concentration of one reactant. It has been suggested that globally fitting all data with single model variable $k_{a}, k_{d}, K_{D}$ and $R_{\max }$ can lead to more consistent kinetic evaluation $^{25}$

\subsection{Results and discussion}

In order to make a comparison between the conventional method for analyzing SPR data and the newly proposed assay procedure, we first analyze the data in the conventional way to calculate the rate and equilibrium constants. Multiple analyte injections of varying analyte concentrations are exposed to a single ligand surface, and an overlay plot is made. The fit to the 1:1 model integrated in Scrubber is shown in Fig. 6.4, and the determined rate and equilibrium constants are presented in Table 6.2. The fitting residuals are shown in units of $\mathrm{m}^{\circ}$, which represent the fit quality. A good fit to the model results in a random residual of minimal $\mathrm{m}^{\circ}$ (Scrubber). 

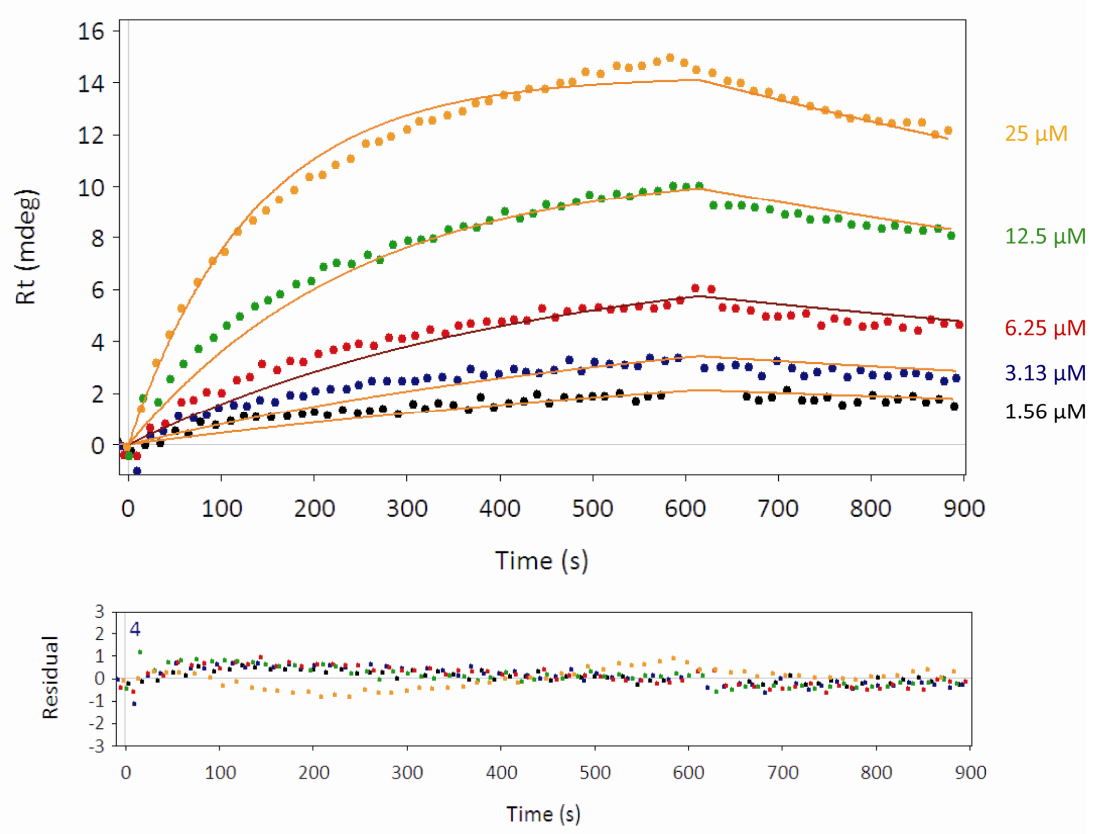

Fig. 6.4. The analyte overlay plot of various analyte concentrations, $25 \mathrm{nM}-1.56 \mathrm{nM}$ in a serial dilution. The global fit results to the 1:1 model integrated in Scrubber, the smooth lines are the fit responses to the measured data in points. The ligand concentration at the surface is $100 \mu \mathrm{M}$. The responses are relatively low due to the combination of ligand and analyte concentration to avoid steric hindrance effects and allow a good fit to the 1:1 model used.

\begin{tabular}{c|cccc} 
Ligand density & $\mathbf{k}_{\mathrm{a}}\left(\mathbf{M}^{-1} \mathbf{s}^{-1}\right)$ & $\mathbf{k}_{\mathrm{d}}\left(\mathbf{s}^{-1}\right)$ & $\mathbf{K}_{\mathrm{D}}(\mathbf{n M})$ & $\mathbf{R}_{\max }\left(\mathbf{m}^{\circ}\right)$ \\
\hline $100 \mu \mathrm{M}$ & $2.14 \times 10^{5}$ & $10.30 \times 10^{-4}$ & 4.78 & 15
\end{tabular}

Table 6.2. The rate and equilibrium constants obtained from the global fit of the analyte overlay plots of at a ligand concentration of $100 \mu \mathrm{M}$.

The exact ligand concentration at the surface is difficult to determine due to the spotting technique used. Therefore, the concentration of the spot-solution is used to indicate the ligand concentration at the surface. These apparently high concentrations, in the micromolar range, are applied in a nanoliter droplet resulting in relatively low ligand concentrations at the surface which are lower compared to the introduced analyte. 
Therefore, the reaction can be described by pseudo-first-order kinetics with the rate equation, (6).

The new assay protocol is based on injecting a single analyte concentration, and exposing it to spots with various ligand densities. In order to compare and determine the rate and equilibrium constants based on various ligand concentrations, the data is also analyzed with Scrubber. The obtained SPR imaging data was analyzed by first loading it into SPRINT (IBIS Technologies B.V., Hengelo, The Netherlands) and selecting the ligand and analyte data to be exported to Scrubber (version 2.0, BioLogic, Campbell, Australia) for further kinetic analysis.

Data obtained from microarray experiments are generally complex due to the large amount of information they contain. This is no exception for SPR imaging data, and an example is shown in Fig. 6.5, where 21 analyte injections were guided over a microarray containing sensor surface.

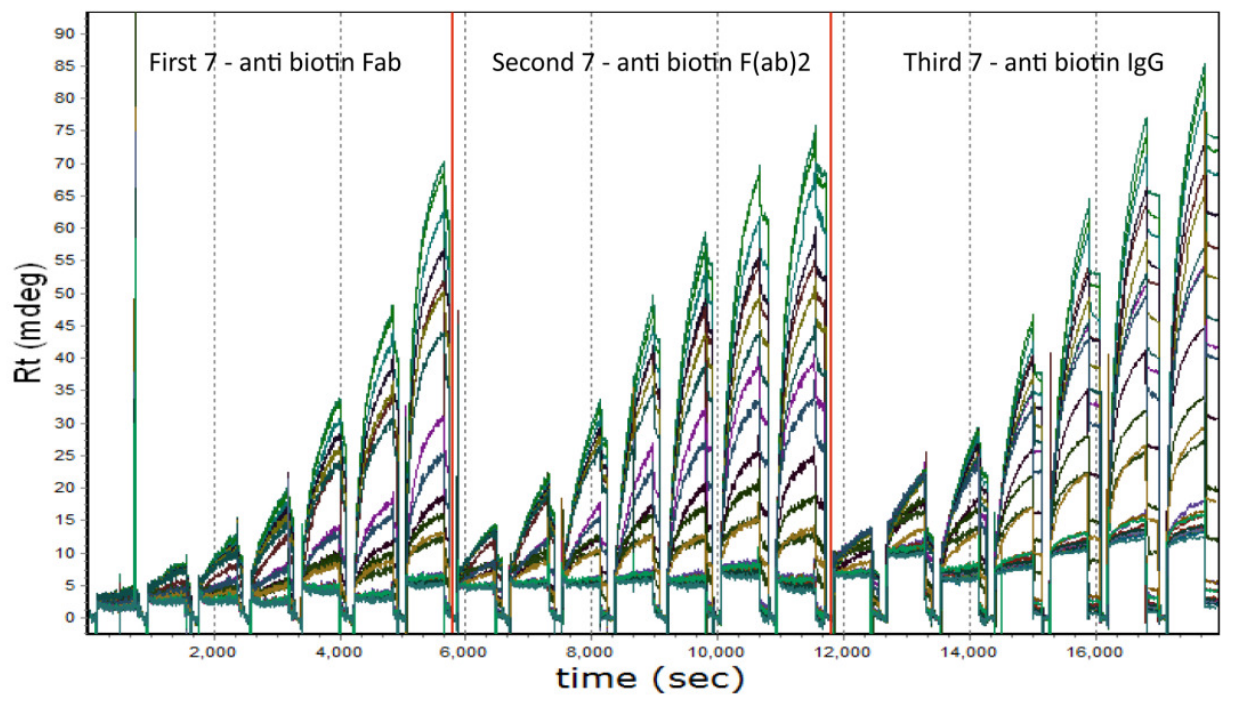

Fig. 6.5. The raw IBIS-iSPR data showing a plot of 525 interactions. 21 injections of various analytes in a serial dilution of $50 \mathrm{nM}$. First 7 interactions are of anti-biotin Fab, second 7 are of anti-biotin $\mathrm{F}(\mathrm{ab})_{2}$ and the third 7 are of anti-biotin IgG. They are on a microarray containing 25 different ligand densities of biotinylated peptides. 
The SPR-microarray for the new assay protocol contains two different sets of ligand dilution series. The first set contains a serial dilution of in ratio-diluted peptides and the second set contains buffer-diluted peptides. This is schematically illustrated in Fig. 6.1, and the SPR image of the obtained microarray positioned in the flow-cell can be seen in Fig. 6.6. The 25 ROls are positioned in the spots of the microarray, here the SPR imaging data is measured.

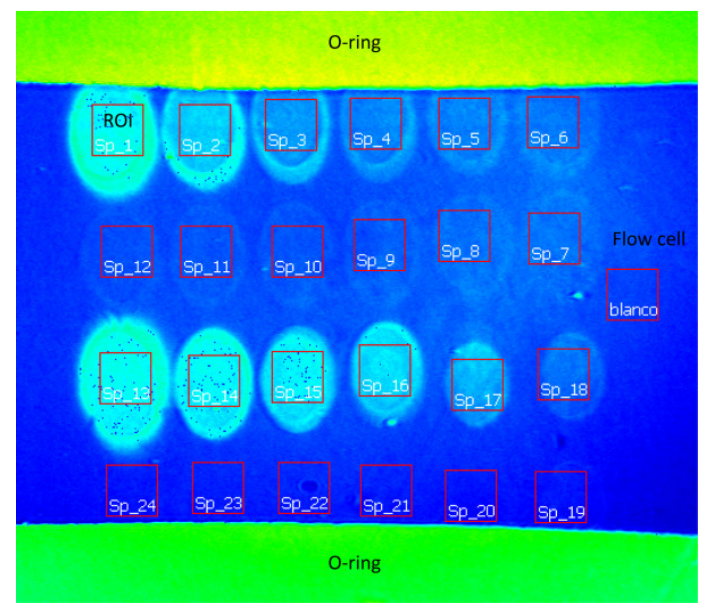

Fig. 6.6. SPR image of a microarray containing serial diluted biotinylated peptides after an interaction with antibiotin in a flow-cell. The ROls (red squares) are positioned within the spots of the microarray. Spot $1-$ Spot 12 represent the 2 fold serial dilution with a non-biotinylated background peptide from $416-0 \mu \mathrm{M}$ (ratio-diluted), and Spot 13 - Spot 24 represent the 2 fold serial dilution with buffer (MES). The color difference of the O-ring (green), protein spots (light blue) and the flow-cell (dark blue), are due to differences in reflectivity due to SPR.

The majority of SPR users use buffer diluted ligands to control the densities on the sensor surface to avoid mass transport limited interactions. Very short immobilization times can contribute to more controlled ligand densities at the sensor surface, however, the exact amounts remain difficult to control ${ }^{15} 16$. In a microarray format the immobilization of the ligands cannot be monitored by following the SPR imaging responses, and therefore, it is more difficult to control the final density at the sensor surface. The spot intensities in Fig. 6.6 indicate the limitations of controlling the ligand density at the surface by means of buffer dilution. Spots 13 through 16 contain nearly the same ligand density due to surface saturation followed by a very quick decrease of ligand in spots 17 and 18, and close to zero 
from spot 19 through 23. And on the other hand, the gradual decrease in intensity at spots 1 through 7 can be seen for the ratio-diluted ligands. The interaction results of 4 ratiodiluted ROI's with 5 analyte injections are shown in a serial plot in Fig. 6.7, these results are part of the results to compare the usability of ligand densities for obtaining kinetic data.

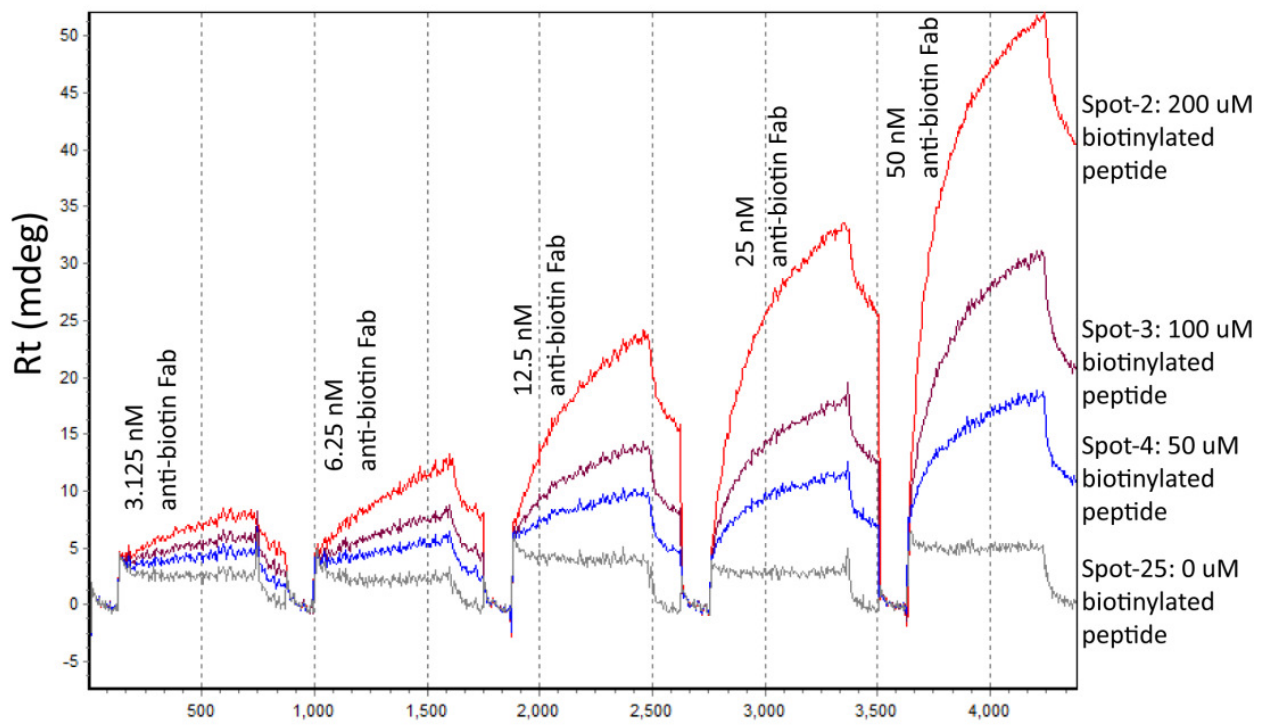

Fig. 6.7. Shows the sensorgrams of sp_2 (red), sp_3 (magenta), sp_4 (blue) and blank (grey) of 5 analyte injections with anti-biotin Fab.

The plots of various ligand densities are made in Scrubber for both, ratio- and bufferdiluted ligands at an analyte concentration of $25 \mathrm{nM}$. The results, shown in Fig. 6.8, show clearly that the ratio-dilution $R_{t}$ intensity varies systematically with the ligand density, however, the buffer-dilution does not follow any particular trend. Table 6.3 shows the comparison between the obtained rate and equilibrium constants of the locally fitted data from the various ligand densities diluted by ratio and buffer, as presented in Fig. 6.8. The extracted $R_{\max }$ value scales linearly with the ligand density at the sensor surface and is therefore an important parameter for evaluating the obtained immobilization results. For the ratio-diluted situation, the surface densities of 25 and $12.5 \mu \mathrm{M}$ appear to be outliers 
as the extracted $k_{a}$ and $k_{d}$ values differ drastically compared to all the other obtained values, and are therefore depicted in red. Further experiments are required to understand this behavior. Therefore, the data of ligand densities, 25 and $12.5 \mu \mathrm{M}$, are excluded from further calculations, and are depicted in red. After excluding 25 and $12.5 \mu \mathrm{M}$, the values of $K_{\mathrm{D}}$ follow the same trend and the extracted values are similar.

\begin{tabular}{|c|c|c|c|c|}
\hline Ratio-diluted & $k_{a}\left(M^{-1} s^{-1}\right)$ & $k_{d}\left(s^{-1}\right)$ & $K_{D}(n M)$ & $R_{\max }\left(m^{\circ}\right)$ \\
\hline $400 \mu \mathrm{M}$ & $2.15 \times 10^{5}$ & $5.90 \times 10^{-4}$ & 2.74 & 48 \\
\hline $200 \mu \mathrm{M}$ & $2.04 \times 10^{5}$ & $6.97 \times 10^{-4}$ & 3.42 & 34 \\
\hline $100 \mu \mathrm{M}$ & $2.17 \times 10^{5}$ & $6.94 \times 10^{-4}$ & 3.19 & 17 \\
\hline $50 \mu \mathrm{M}$ & $2.50 \times 10^{5}$ & $5.84 \times 10^{-4}$ & 2.30 & 9 \\
\hline $25 \mu \mathrm{M}$ & $3.50 \times 10^{5}$ & $10.41 \times 10^{-4}$ & 3.00 & 7 \\
\hline $12.5 \mu \mathrm{M}$ & $8.00 \times 10^{5}$ & $10.60 \times 10^{-4}$ & 1.30 & 4 \\
\hline $6.25 \mu \mathrm{M}$ & $2.70 \times 10^{5}$ & $3.20 \times 10^{-4}$ & 1.20 & 1 \\
\hline buffer-diluted & $k_{a}\left(M^{-1} s^{-1}\right)$ & $k_{d}\left(s^{-1}\right)$ & $K_{D}(n M)$ & $\mathbf{R}_{\max }\left(\mathbf{m}^{\circ}\right)$ \\
\hline $400 \mu \mathrm{M}$ & $2.24 \times 10^{5}$ & $5.70 \times 10^{-4}$ & 2.55 & 47 \\
\hline $200 \mu \mathrm{M}$ & $2.27 \times 10^{5}$ & $5.90 \times 10^{-4}$ & 2.60 & 43 \\
\hline $100 \mu \mathrm{M}$ & $2.41 \times 10^{5}$ & $5.80 \times 10^{-4}$ & 2.42 & 38 \\
\hline $50 \mu \mathrm{M}$ & $2.65 \times 10^{5}$ & $6.00 \times 10^{-4}$ & 2.28 & 34 \\
\hline $25 \mu \mathrm{M}$ & $2.88 \times 10^{5}$ & $6.60 \times 10^{-4}$ & 2.29 & 29 \\
\hline $12.5 \mu \mathrm{M}$ & $2.90 \times 10^{5}$ & $6.10 \times 10^{-4}$ & 2.10 & 13 \\
\hline $6.25 \mu \mathrm{M}$ & $2.10 \times 10^{5}$ & $6.00 \times 10^{-4}$ & 1.90 & 6 \\
\hline
\end{tabular}

Table 6.3. The rate and equilibrium constants obtained from the ratio- and buffer-diluted ligand-overlay plots presented in Fig. 6.8.

When comparing the extracted $k_{a}$ and $k_{d}$ values of the two dilution methods, ratio and buffer, a clear similarity can be found. The $k_{a}$ and $k_{d}$ values of the ratio-dilution are, $2.31 \mathrm{x}$ $10^{5} \pm 0.28$ and $5.77 \times 10^{-4} \pm 1.54$, and the $k_{a}$ and $k_{d}$ values of the buffer-dilution are, $2.49 \times$ $10^{5} \pm 0.32$ and $6.01 \times 10^{-4} \pm 0.29$, respectively. The kinetic rate and equilibrium constants obtained by the two different analyses procedures, are not completely identical, however, within the same order of magnitude. The extracted $k_{a}$ and $k_{d}$ obtained from the analyte overlay plot, listed in Table 6.2, show slight differences with $2.1 \times 10^{5}$ and $10.3 \times 10^{-4}$, compared the values derived from Table 6.3. However, the values should be compared to the $100 \mu \mathrm{M}$ ratio-diluted ligand density, which are $2.2 \times 10^{5}$ and $6.9 \times 10^{-4}$. 

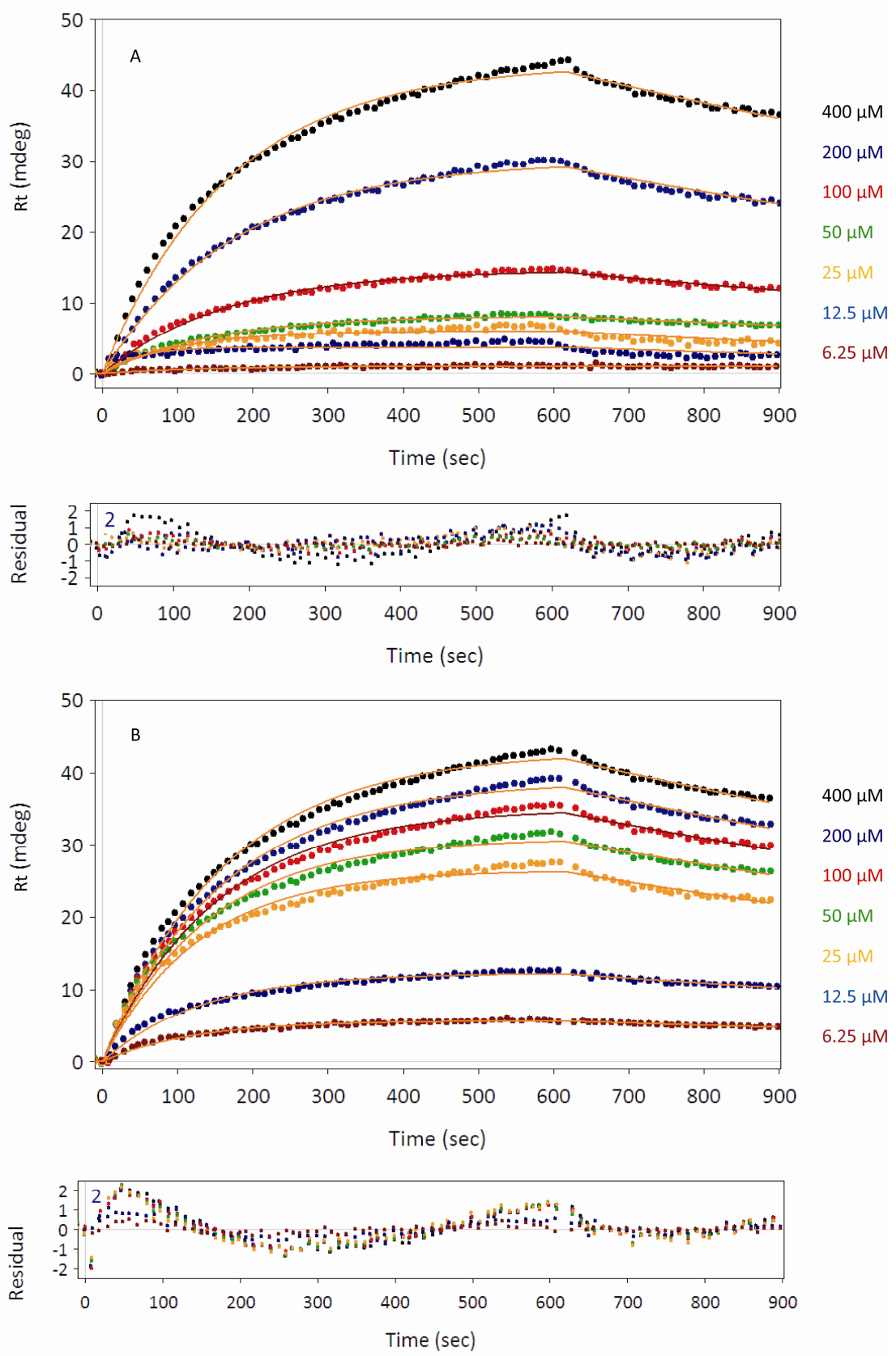

Fig. 6.8. The results of the RATIO-diluted (A) and buffer-diluted (B) ligand concentrations with overlays of various ligand concentrations, $400 \mu \mathrm{M}-25 \mu \mathrm{M}$ and an analyte concentration $25 \mathrm{nM}$. The smooth lines are the local fit responses to the measured data in points. 
Nevertheless, the preliminary data suggests that the new method based on various ligand densities at the surface, instead of various analyte concentrations produces similar kinetic information, and may be further explored as an assay protocol as it requires fewer regeneration steps and reduces the overall assay time. The similarity in values is expected, as the studied interaction always relates to the biotinylated peptide immobilized on the surface and the anti-biotin Fab as analyte. Furthermore, the 1:1 model used in scrubber, a pseudo-first-order algorithm, only considers one concentration range, in this case the analyte and not the ligand. The extracted $k_{a}$ and $k_{d}$ values of the buffer-diluted ligand densities are behaving more similarly to the ratio-diluted ligand densities as expected. The effect of the ratio-dilution seems therefore negligible compared to the buffer-dilution. Most interesting is the clear effect the new dilution method has on $R_{\max }$. The results, listed in table 6.3, show the differences in $R_{\max }$, and are plotted against the ligand density in Fig. 6.9, to determine the correlation and accuracy of the dilution method used. From Fig. 6.9, the least square fits to the extracted $\mathrm{R}_{\max }$ values for both ratio-dilution and buffer-dilution experiments are shown. Coincidentally, the slopes of the lines are the same $\approx 0.16 \mathrm{~m} /[B]$ $\mu \mathrm{M}$, however, the $\mathrm{R}^{2}$ of the ratio-diluted fit is 0.99 compared to 0.64 for the buffer-diluted fit indicating a much better correlation to a linear increase in $[B]$. The $R_{\max }$ offset value of 1.36 , in the case of ratio-diluted, compared to 16.83 , in the case of buffer-diluted, are an indication that the ratio-dilution approaches the ideal value of 0 compared to the bufferdilution. The linear correlation between the ligand density of the ratio-dilution, further supports that the ratio dilution approach is a more controlled method of manipulating the ligand density at the sensor surface. The extracted $R_{\text {max }}$, from Scrubber, is compared with the calculated $R_{\max }$ from the least square fit. The relative error is used to compare the $R_{\max }$ with the ideal response values. Very low values for the relative error indicates a high accuracy of the estimated ligand density at the surface compared to the actual ligand density at the surface. In the case of ratio-dilution, the relative error are less than $5 \%$, except the 400, 25 and $12.5 \mu \mathrm{M}$. The buffer-dilution on the other hand, ranges from $12 \%$ to over $66 \%$. The increased relative error for the $400 \mu \mathrm{M}$ ligand concentration is caused by the saturation of the surface for this given ligand density. The calculated maximal surface density at a $R_{\max }$ value of $48 \mathrm{~m}^{\circ}$, is $300 \mu \mathrm{M}$. 


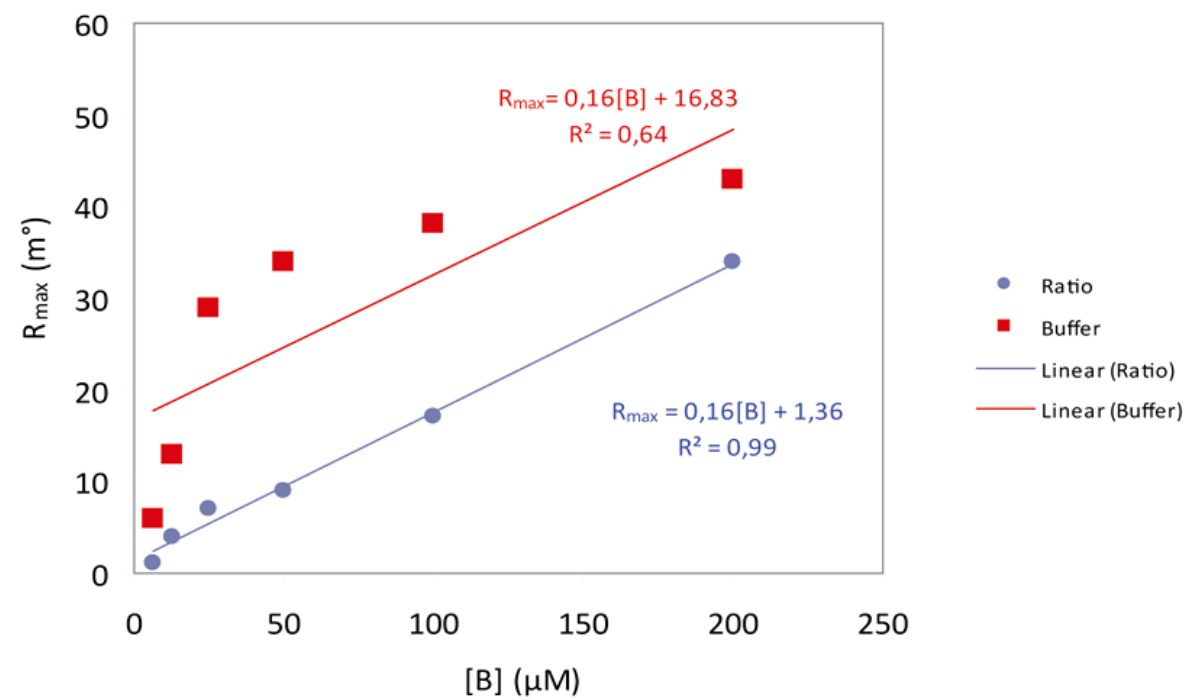

Fig. 6.9. Comparison of least square fit to the extracted $R_{\max }$ values between the ratio-dilution and buffer-dilution experiments with variable immobilized ligand concentrations [B] by an analyte concentration of $25 \mathrm{nM}$.

\begin{tabular}{|c|c|c|c|c|c|c|}
\hline $\begin{array}{c}\text { [B] } \\
\text { buffer }\end{array}$ & $\begin{array}{c}\text { Calculated } \\
\mathbf{R}_{\max } \\
\text { (ratio) }\end{array}$ & $\begin{array}{c}\text { Extracted } \\
\mathbf{R}_{\max } \\
\text { (ratio) }\end{array}$ & $\begin{array}{c}\text { Relative } \\
\text { error \% } \\
\text { (ratio) }\end{array}$ & $\begin{array}{c}\text { Calculated } \\
\mathbf{R}_{\max } \\
\text { (buffer) }\end{array}$ & $\begin{array}{c}\text { Extracted } \\
\mathbf{R}_{\max } \\
\text { (buffer) }\end{array}$ & $\begin{array}{c}\text { Relative } \\
\text { error \% } \\
\text { (buffer) }\end{array}$ \\
\hline $400 \mu \mathrm{M}$ & 65.4 & 48 & 27 & 80.8 & 47 & 41.8 \\
\hline $200 \mu \mathrm{M}$ & 33.4 & 34 & 1.8 & 48.8 & 43 & 11.9 \\
\hline $100 \mu \mathrm{M}$ & 17.4 & 17 & 2.3 & 32.8 & 38 & 15.7 \\
\hline $50 \mu \mathrm{M}$ & 9.4 & 9 & 4.2 & 24.8 & 34 & 37.1 \\
\hline $25 \mu \mathrm{M}$ & 5.4 & 7 & 29.6 & 20.8 & 29 & 39.4 \\
\hline $12.5 \mu \mathrm{M}$ & 3.4 & 4 & 17.6 & 18.8 & 13 & 30.9 \\
\hline $6.25 \mu \mathrm{M}$ & 2.4 & 1 & 58.3 & 17.8 & 6 & 66.3 \\
\hline
\end{tabular}

Table 6.4: Represents the relative error between the extracted $R_{\max }$ and the calculated $R_{\max }$ derived from the least square fit of the ratio-dilution and buffer-dilution experiments.

Another method to compare the ligand immobilization protocol is by measuring the fluorescence intensity, see Figs. 6.10a and b. The same experimental conditions were used to print the microarray on a new sensor surface, and an excess of analyte was introduced for 1 hour to saturate the surface. 

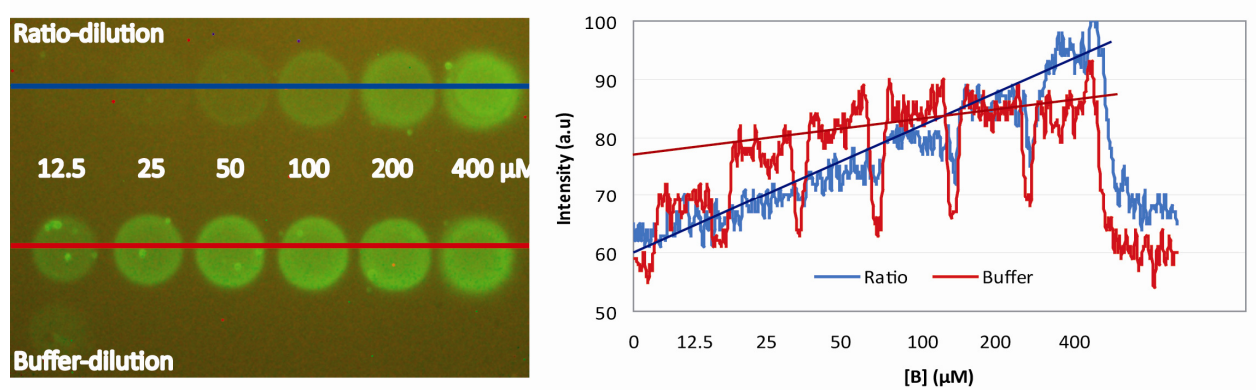

Fig. 6.10a. Fluoresence image of the microarray with ratio- and buffer-diluted biotinylated ligands immobilized at the surface, after the interaction with $67 \mathrm{nM}$ anti-biotin Alexa fluor 488. The red and blue lines resemble the position from where the data for the fluorescence intensity plot is taken. The blue line resembles the ratiodiluted ligands, and red the buffer-diluted ligands. Fig.6.10b. The fluorescence intensity plot derived from the fluorescence image. The straight lines indicate the trend of the fluorescence intensity with [B]. The fluorescence intensity of the ratio-diluted (blue) ligands scales with [B], the fluorescnece intensity of the buffer-diluted (red) ligands initially remains high and then drops rapidly.

To compare the fluorescence intensity plot with the obtained SPR imaging results $\left(R_{\max }\right)$, normalized signals with a maximum of unity were used and plotted in Figs. 6.11a and b. The average fluorescence intensities were determined for the different ligand concentrations used. The correlation between the obtained SPR and fluorescence results indicate similar behavior between the two methods is evidenced by similar correlation coefficients. The least square fit of the ratio-dilution is linear while the least square fit of the buffer-dilution is logarithmic, indicating a clear difference between the two dilution methods used. The slight deviations between the fluorescence and SPR results are caused by variations of sensor discs and experimental conditions. In both cases, SPR imaging and fluorescence, an excess of analyte was used compared to the ligand at the surface. $100 \mu \mathrm{l}$ of $25 \mathrm{nM}$ and $250 \mu \mathrm{l}$ of $67 \mathrm{nM}$ analyte concentration, compared to $1 \mathrm{nl}$ droplets of 1 - 400 $\mu \mathrm{M}$ ligand concentration. The used concentrations result in at least 5 times more analyte molecules compared to ligand molecules. This is necessary to visualize the biotinylated ligand molecules present at the surface without limitation due to the analyte. 


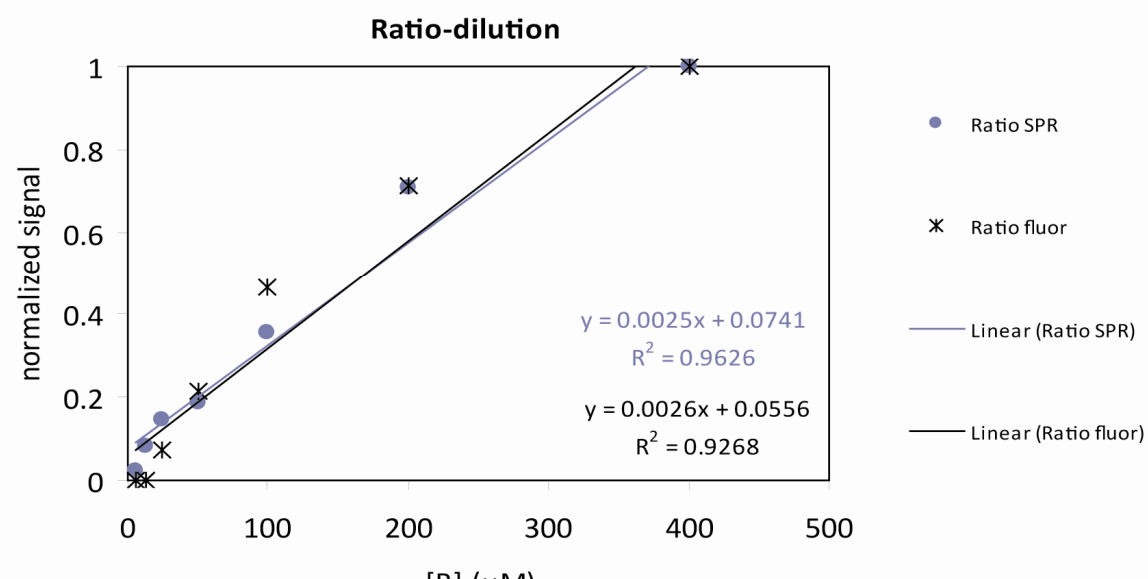

[B] $(\mu \mathrm{M})$

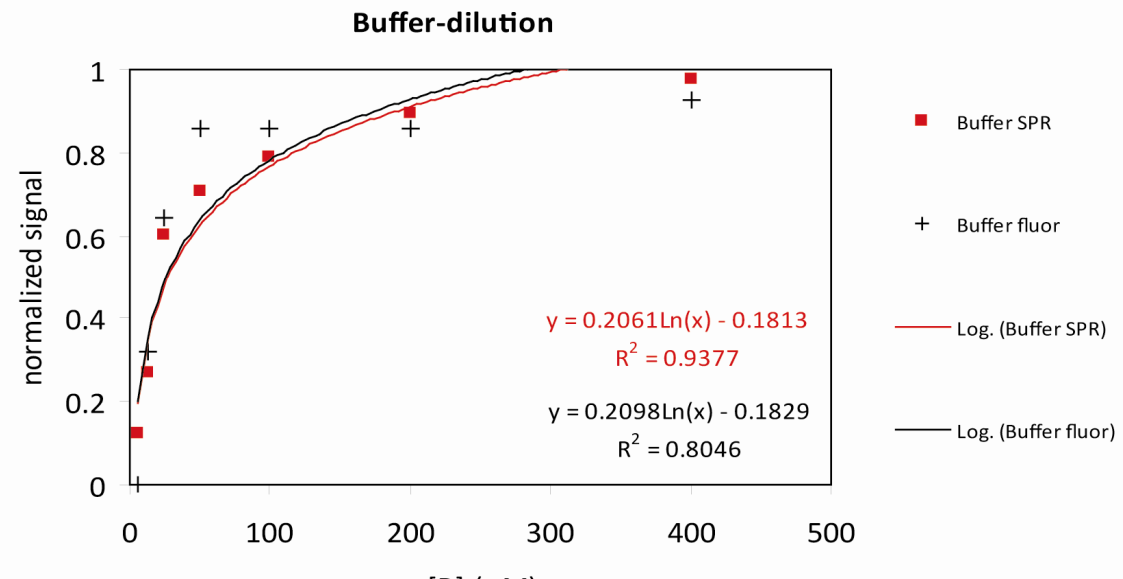

[B] $(\mu \mathrm{M})$

Fig. 6.11a. The ratio-diluted ligand immobilization profile, depicted in normalized signals to compare fluorescence intensity profiles with and SPR imaging results. The fluorescence measurements are depicted in black, and SPR imaging in blue. The analyte concentration used are, $25 \mathrm{nM}$ for the SPR imaging experiment and $67 \mathrm{nM}$ for the fluorescence intensity experiment. Fig. 6.11b. The buffer-diluted ligand immobilization profile, depicted in normalized signals to compare fluorescence intensity profiles with and SPR imaging results. The fluorescence measurements are depicted in black, and SPR imaging in red. 
Multivalency, conformational changes, steric hindrance, mass transport limitations, rebinding due to surface geometry and surface heterogeneity are all known factors affecting the fit to the simple $1: 1$ model $^{10}$. Here different anti-biotin analytes were tested, Fab, $F(a b)_{2}$, and IgG to get additional insight of the binding process in relation to the ligand density. The $50 \mathrm{kDa}$ Fab can most likely be described with the 1:1 interaction model as it is a small monovalent molecule. The $\mathrm{F}(\mathrm{ab})_{2}$ and $\operatorname{lgG}$ on the other hand are bivalent molecules, with molecular weights of 100 and $150 \mathrm{kDa}$, respectively. The differences in behavior can be nicely observed in the ratio-diluted interactions comparing the $200 \mu \mathrm{M}$ and $50 \mu \mathrm{M}$ densities shown in Fig. 6.12. and are listed in Table 6.5.

\begin{tabular}{|c|c|c|c|c|}
\hline $200 \mu \mathrm{M}$ ligand & $k_{a}\left(M^{-1} s^{-1}\right)$ & $k_{d}\left(s^{-1}\right)$ & $\mathrm{K}_{\mathrm{D}}(\mathrm{nM})$ & $R_{\max }\left(\mathrm{m}^{\circ}\right)$ \\
\hline Fab & $1.82 \times 10^{5}$ & $9.70 \times 10^{-4}$ & 5.30 & 37 \\
\hline $\mathrm{F}(\mathrm{ab})_{2}$ & $2.27 \times 10^{5}$ & $1.10 \times 10^{-4}$ & 0.47 & 49 \\
\hline $\lg G$ & $2.27 \times 10^{5}$ & $0.50 \times 10^{-4}$ & 0.22 & 52 \\
\hline $50 \mu \mathrm{M}$ ligand & $k_{a}\left(M^{-1} s^{-1}\right)$ & $k_{d}\left(s^{-1}\right)$ & $\mathrm{K}_{\mathrm{D}}(\mathrm{nM})$ & $R_{\max }\left(m^{\circ}\right)$ \\
\hline $\mathrm{Fab}$ & $2.49 \times 10^{5}$ & $7.08 \times 10^{-4}$ & 2.84 & 9 \\
\hline $\mathrm{F}(\mathrm{ab})_{2}$ & $2.36 \times 10^{5}$ & $8.77 \times 10^{-4}$ & 3.72 & 21 \\
\hline $\lg G$ & $3.49 \times 10^{5}$ & $5.37 \times 10^{-4}$ & 1.54 & 29 \\
\hline
\end{tabular}

Table 6.5: The rate and equilibrium constants obtained from the monovalent Fab, and bivalent $F(a b)_{2}$ and $\operatorname{lgG}$ analytes and their behavior on the ligand density.

The $\mathrm{F}(\mathrm{ab})_{2}$ and IgG responses are fairly similar for the $200 \mu \mathrm{M}$ ligand density, despite their difference in mass. This could be a sign of accessibility / steric hindrance or mass transport problems in the hydrogel matrix used for the experiment. Furthermore, the bivalency contributes to a lower dissociation rate due to the fact that the analyte is bound to two ligand molecules at the surface. The chance of both bonds breaking simultaneously is less compared to the monovalent bond. This can also be concluded of the decreased value of the dissociation rate constant, $k_{d}$, and equilibrium constant $K_{D}$, obtained for the bivalent molecules in the $200 \mu \mathrm{M}$ situation. The extracted $k_{d}$ and $K_{D}$ value of the $F(a b)_{2}$ and IgG, significantly different from the Fab, are $\approx 10$-fold smaller. 

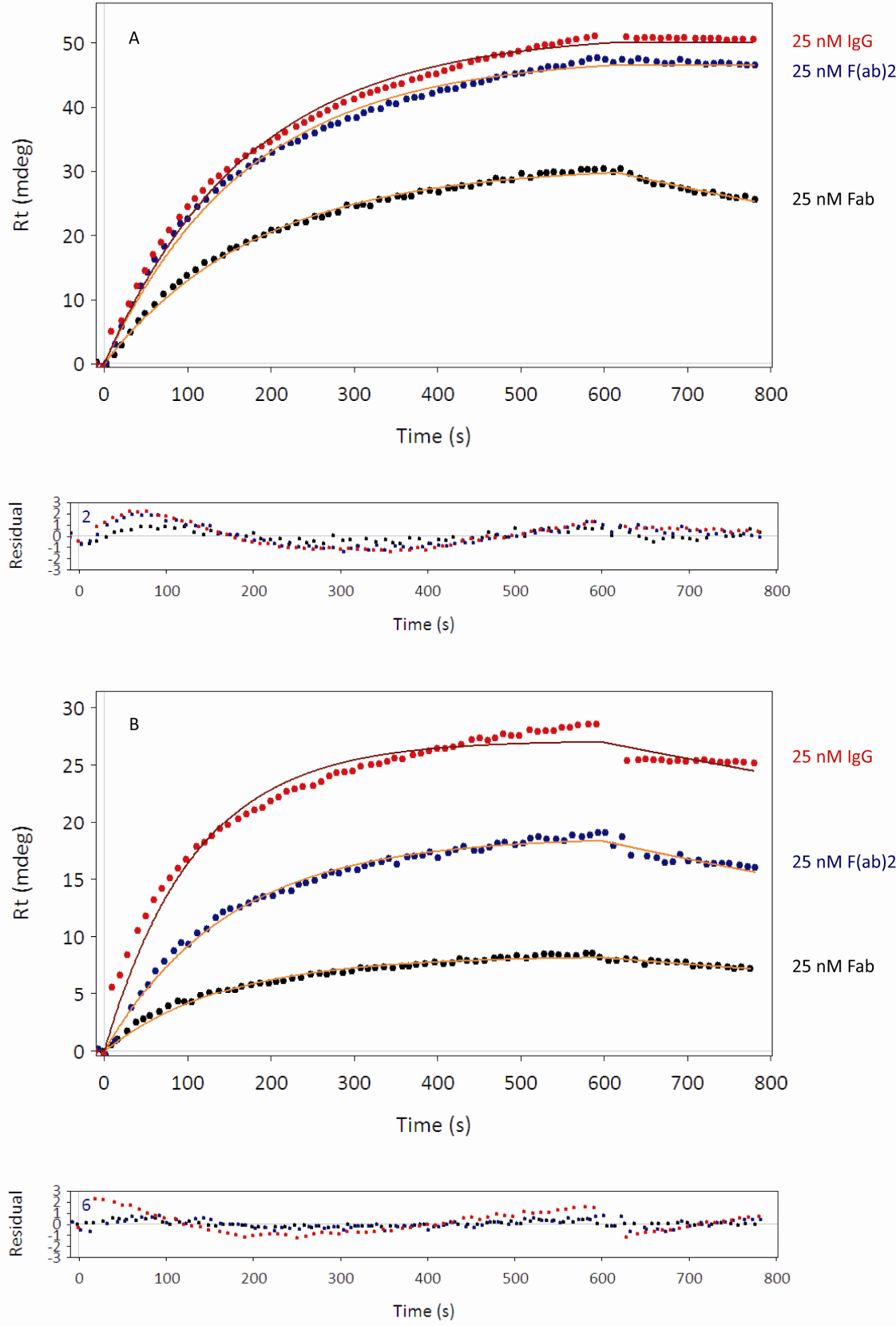

Fig. 6.12. Overlay of IgG, F(ab)2 and Fab analyte at 2 different ligand concentrations, $200 \mu \mathrm{M}(\mathrm{A})$ and $50 \mu \mathrm{M}$ (B) and with an analyte concentration of $25 \mathrm{nM}$. The smooth lines are the fit responses to the measured data. 
The extracted values for the rate and equilibrium constants obtained at the low ligand density surface, $50 \mu \mathrm{M}$, are all within the same order of magnitude. Due to the decreased ligand density, the various analytes all behave in a monovalent manner. The $\mathrm{Rt}_{(600 \mathrm{sec})}$ obtained at the $50 \mu \mathrm{M}$ ligand density surface are: $\mathrm{Fab}=8.5 \mathrm{~m}^{\circ}, \mathrm{F}(\mathrm{ab})_{2}=19.0 \mathrm{~m}^{\circ}$ and $\lg \mathrm{g}=$ $28.5 \mathrm{~m}^{\circ}$, approximately proportional to the ratio of molecular masses of 50, 100 and 150 $\mathrm{kDa}$. The concentration of the ligand density at the sensor surface clearly contributes to the fitting to the 1:1 model, where bivalent molecules behave like monovalent molecules. From the obtained results we can conclude that by applying the ratio-diluted method, the ligand density at the surface is much better controlled compared to the buffer-diluted method. Moreover the spacing of the bio-active ligand molecule is increased, a technique commonly used in making self assembled monolayer's (SAM) ${ }^{32}$. The use of universal background molecules has to be further investigated, here we used a non biotinylated peptide with the same amino acid sequence to eliminate different binding kinetics as a background molecule.

\subsection{Conclusion}

Immobilization kinetics play an essential role in the actual ligand density at the surface when diluted with buffer. In an attempt to control the ligand density on the surface, background molecules were added to the ligand solution. By maintaining a constant surface molecule density, a precise control of ligand density can be achieved, as demonstrated with linear variation of $R_{\max }$ extracted from measured responses. The difference in ligand density at the surface due to the dilution procedure used, can be seen in Fig. 6.9 by the difference in the least square fit, and the relative error presented in Table 6.4. Another observation made by reducing the ligand density at the surface, is related to the molecular binding behavior of the analyte. The binding responses of monovalent and bivalent analytes were compared at surfaces containing various ligand densities. At high $(200 \mu \mathrm{M})$ ligand densities, the bivalent molecules form a stronger double bond with the ligand as can be seen by the poor dissociation properties. At low $(50 \mu \mathrm{M})$ ligand densities on the other hand, the bivalent molecules behave like monovalent molecules, because they cannot bind two ligand molecules at the same time due to spacial distribution. The observations are presented in Fig. 6.12, and Table 6.5. 
One of the reasons to perform this study was to compare the conventional assay protocol with the single analyte kinetic analysis technique. The results shown in this chapter are encouraging where a clear linear trend with extracted $R_{\max }$ values, which scale proportionally with the ligand concentration [B]. As stated earlier, this new assay protocol technique has clear advantages in requiring much smaller analyte volumes and drastically reduced surface regeneration steps, and also lead to reduced assay times and costs. Other ligand and analyte model systems need to be investigated to show the general applicability to the scientific community. The controlled ligand density is not only important for kinetic analysis, other assays can also benefit from this new assay protocol.

\subsection{References}

$1 \quad$ Liedberg, B.; Nylander, C.; Lundstrom, I., Surface plasmon resonance for gas detecting and biosensing. Sensors and Actuators B chemical 4, 299-304 (1983).

2 Rothenhauserler, B.; Knoll, W., Surface plasmon microscopy. Nature 332, 615-617 (1988)

3 Schasfoort, R.B.M.; Tudos, A.J., Handbook of surface plasmon resonance. (The Royal Society of Chemistry, Cambridge, UK, 2008).

4 Homola, J.; Yee, S.S.; Gauglitz, G., Surface plasmon resonance sensors: review. Sensors and Actuators B chemical 54, 3-15 (1999).

5 Homola, J.; Vaisocherová, H.; Dostálek, J.; Piliarik, M., Multi-analyte surface plasmon resonance biosensing. Methods 37 (1), 26-36 (2005).

Karlsson, R.: Michaelsson, A.; Mattsson, L., Kinetic analysis of monoclonal antibody-antigen interactions with a new biosensor based analytical system. Journal of Immunological Methods 145, 229-240 (1991).

7 Rich, R.L.; Myszka, D.G., Survey of the year 2005 commercial optical biosensor literature. Journal of Molecular Recognition 19, 478-534 (2006).

8 Karlsson, Robert, SPR for molecular interaction analysis: a review of emerging application areas. Journal of Molecular Recognition 17 (3), 151 (2004). 

throughput analyses of biomolecular binding events and their kinetics. Biomaterials 28, 2380-2392 (2007). Myszka, D.G., Kinetic analysis of macromolecular interactions using surface plasmon resonance biosensors. Current Opinion in Biotechnology 8, 50-57 (1997).

11 Trutnau, H.-H., New multi-step kinetics using common affinity biosensors saves time and sample at full access to kinetics an concentration. Journal of Biotechnology 124, 191-195 (2006).

12 Karlsson, R.; Katsamba, P.S.; Nordin, H.; Pol, E.; Myszka, D.G., Analyzing a kinetic titration series using affinity biosensors. Analytical Biochemistry 349, 136-147 (2006).

13 Bravman, T.; Bronner, V.; Lavie, K.; Notcovich, A.; Papalia, G.A.; Myszka, D.G., Exploring "one-shot' kinetics and small molecule analysis using the ProteOn XPR36 array biosensor. Analytical Biochemistry 358, 281-288 (2006). Abdiche, Y.; Malashock, D.; Pinkerton, A.; Pons, J., Determining kinetics and affinities of protein interactions using a parallel real-time label-free biosensor, the Octet. Analytical Biochemistry 377 (2), 209-217 (2008). Katsamba, P. S.; Navratilova, I.; Calderon-Cacia, M.; Fan, L.; Thornton, K.; Zhu, M.; Bos, T. Vanden; Forte, C.; Friend, D.; Laird-Offringa, I.; Tavares, G.; Whatley, J.; Shi, E.; Widom, A.; Lindquist, K.C.; Klakamp, S.; Drake, A.; Bohmann, D.; Roell, M.; Rose, L.; Dorocke, J.; Roth, B.; Luginbühl, B.; Myszka, D.G., Kinetic analysis of a high-affinity antibody/antigen interaction performed by multiple Biacore users. Analytical Biochemistry 352 (2), 208221 (2006).

Navratilova, I.; Papalia, G.A.; Rich, R.L.; Bedinger, D.; Brophy, S.; Condon, B.; Deng, T.; Emerick, A.W.; Guan, H.-W.; Hayden, T.; Heutmekers, T.; Hoorelbeke, B.; McCroskey, M.C.; Murphy, M.M.; Nakagawa, T.; Parmeggiani, F.; Qin, X.; Rebe, S.; Tomasevic, N.; Tsang, T.; Waddell, M.B.; Zhang, F.F.; Leavitt, S.; Myszka, D.G., Thermodynamic benchmark study using Biacore technology. Analytical Biochemistry 364 (1), 67-77 (2007). 
17 Elbs, M.; Hulko, M.; Frauenfeld, J.; Fisher, R.; Brock, Multivalence and spot heterogeneity in microarray-based measurement of binding constants. Analytical and Bioanalytical Chemistry 387, 2017-2025 (2007). Rich, R.L.; Myszka, D.G., Higher-throughput, label-free, real-time molecular interaction analysis. Analytical Biochemistry 361, 1-6 (2007).

19 Rich, R.L.; Cannon, M.J.; Jenkins, J.; Pandian, P.; Sundaran, S.; Magyar, R.; Brockman, J.; Lambert, J.; Myszka, D.G., Extracting kinetic rate constants from surface plasmon resonance array systems. Analytical Biochemistry 373, 112-120 (2008).

20 Johnsson, B.; Löfås, S.; Lindquist, G., Immobilization of proteins to a carboxymethyldextran-modified gold surface for biospecific interaction analysis in surface plasmon resonance sensors. Analytical Biochemistry 198 (2), 268-277 (1991).

21 Stigter, E.C.A.; Jong, G.J. de; van Bennekom, W.P., An improved coating for the isolation and quantitation of interferon-[gamma] in spiked plasma using surface plasmon resonance (SPR). Biosensors and Bioelectronics 21 (3), 474482 (2005).

22 de Heij, B.; Daub, M.; Gutmann, O.; Niekrawietz, R.; Sandmaier, H.; Zengerle, R., Highly parallel dispensing of chemical and biological reagents. Analytical and Bioanalytical Chemistry 378 (1), 119-122 (2004).

23 Beusink, J.B.; Lokate, A.M.C.; Besselink, G.A.J.; Pruijn, G.J.M.; Schasfoort, R.B.M., Angle-scanning SPR imaging for detection of biomolecular interactions on microarrays. Biosensors and Bioelectronics 23 (6), 839-844 (2008).

24 Lokate, A.M.C.; Beusink, J.B.; Besselink, G.A.J.; Pruijn, G.J.M.; Schasfoort, R.B.M., Biomolecular Interaction Monitoring of Autoantibodies by Scanning Surface Plasmon Resonance Microarray Imaging. Journal of the American Chemical Society 129 (45), 14013-14018 (2007).

25 Roden, L.D.; Myszka, D.G., Global analysis of a macromolecular interaction measured on BIAcore. Biochemical and Biophysical Research Communications 225 (3), 1073-1077 (1996). 
26 Schuck, P., Use of surface plasmon resonance to probe the equilibrium and dynamic aspects of interactions between biological macromolecules. Annual Review Biophysical Biomolecular Structure 26, 541-566 (1997).

27 O'Shannessy, D.J., Determination of kinetic rate equilibrium binding constants for macromolecular interactions: a critique of surface plasmon resonance literature. Current Opinion in Biotechnology 5, 65-71 (1994).

Oshannessy, D.J.; Brighamburke, M.; Soneson, K.K.; Hensley, P.; Brooks, I., Determination of Rate and Equilibrium Binding Constants for Macromolecular Interactions Using Surface Plasmon Resonance: Use of Nonlinear Least Squares Analysis Methods. Analytical Biochemistry 212 (2), 457-468 (1993).

29 Edwards, P.R.; Leatherbarrow, R.J., Determination of Association Rate Constants by an Optical Biosensor Using Initial Rate Analysis. Analytical Biochemistry 246 (1), 1-6 (1997).

30 Myszka, D.G.; Morton, T.A.; Doyle, M.L.; Chaiken, I.M., Kinetic analysis of a protein antigen-antibody interaction limited by mass transport on an optical biosensor. Biophysical Chemistry 64 (1-3), 127-137 (1997).

31 Joss, L.; Morton, T.A.; Doyle, M.L.; Myszka, D.G., Interpreting Kinetic Rate Constants from Optical Biosensor Data Recorded on a Decaying Surface. Analytical Biochemistry 261 (2), 203-210 (1998).

32 Wirth, M.J.; Fairbank, R.W.P.; Fatunmbi, H.O., Mixed Self-Assembled Monolayers in Chemical Separations. Science 275 (5296), 44-47 (1997). 



\section{Conclusions \& recommendations}

This chapter provides an overview of the material presented throughout this thesis, and provides recommendations for improvement of the equipment, instrumentation and assay procedures. 


\subsection{Conclusions}

In this thesis the use of a peptide microarray in combination with SPR imaging for the detection of autoantibodies present in serum samples were explored, to provide a labelfree alternative to conventional assays, such as ELISA. The need for parallelized testing, to enlarge the obtainable information from a single test, is increasing. On the other hand testing should become as cheap as possible, labeled antibodies are costly to produce and moreover they increase the assay time. Ligand density is very important for the visualization of the microarray and also the performance of the assay.

Microarrays can be made in various ways. Here was decided to make them with a commercial instrument (TopSpot), which uses a piezoactuator to dispense the ligands on the surface. The obtained spot sizes are mainly dependent on the fluid and surface properties, however, the velocity of actuation also plays a role. The $1 \mathrm{nl}$ droplets dry easily, and when handling fragile ligands a fast transfer to an humidity chamber is essential to avoid drying or even denaturation. In an attempt to make a disposable spotting device which also results in confined spot sizes and provides a friendly environment for the ligands, various microfluidic geometries were studied in PDMS. A device merely driven by capillary forces to immobilize the ligands on the surface resulted in a line-spotter and a stop-valve. The geometry and size limitations of the sensor disc and flow-cell, however, do not allow for high density microarrays.

The analyte binding behavior is influenced by the ligand density at the sensor surface. At high ligand densities, bivalent molecules can form a (stronger) double interaction with the ligand. At low ligand densities on the other hand, bivalent molecules behave like monovalent molecules. These observations were confirmed by the differences in dissociation properties. Immobilization kinetics plays an essential role in the actual ligand density at the surface when diluted with buffer. In an attempt to control the total ligand density on the surface, molecules were mixed with the ligand solution to keep a constant total concentration. A precise control of ligand density can be achieved, as demonstrated with linear variation of $R_{\max }$ extracted from measured responses for the ratio-diluted method. Comparison of the conventional assay protocol with the single analyte kinetic analysis technique are encouraging having a clear linear trend with extracted $R_{\max }$ values, scaling proportionally with the ligand concentration. Microarrays can be constructed more efficiently when ratio-dilution is applied. Moreover, this new assay protocol technique has 
clear advantages in requiring much smaller analyte volumes and drastically reduced surface regeneration steps leading to reduced assay times and costs.

The scanning-angle SPR imaging system used throughout this thesis, is able to measure many different biomolecular interactions simultaneously at an imaging area of $50 \mathrm{~mm}^{2}$. By measuring the angle-shift of the SPR-dip, it is a reliable and sensitive measurement with increased linearity compared to fixed-angle SPR systems. Due to the wide range of operation of the instrument both peptide and protein interactions can be measured simultaneously. The amount of IgG molecules bound to the surface and detected in a label-free manner is $4.1 \times 10^{4}$ molecules or 65 zeptomole. The effectiveness of this system is demonstrated by automatically monitoring the interactions between citrullinated peptides and serum autoantibodies of 50 rheumatoid arthritis (RA) patients and 29 controls in a single step. The smallest antibody concentration that could be measured in this experimental setup was $0.5 \mathrm{pM}$, which is slightly less sensitive compared to the ELISA test. ELISA only allows for separate analysis for each type of antibodies to be tested, whereas SPR microarray imaging facilitates multiplexing in a label-free manner.

\subsection{Recommendations}

There are various aspects of the here performed research that raised new questions or just need further investigation. The recommendations are discussed here per subject.

\subsubsection{Microarray technology}

As mentioned before, the geometry of the PDMS stop-valves does not allow for the production of high density microarrays. In order to meet researchers needs, pressure driven devices seem inevitable when using native or unaltered PDMS. Altering the fluid properties, by lowering the surface tension could facilitate easier filling of the channels, however, might also induce leakage at the nozzles. Other materials should be investigated in order to find a fast and cheap alternative for the PDMS and glass chips.

PDMS is very suitable for patterning a surface by micro-contact printing by means of adsorption at the surface by van der Waals forces. By creating hydrophilic islands, surrounded by a hydrophobic surface, droplets of ligands can be covalently coupled at the surface during immobilization. The excess of ligand can be simply washed away, the 
hydrophobic coating needs to be removed by a pH or salt shock / injection prior to the SPR imaging experiment to avoid disturbance of the analyte flow in the flow-cell.

A technique similar to that of loading an electrophoresis gel could be applied to avoid drying of the droplets. Tiny cups submerged under a buffer could be filled with ligand to be immobilized at the surface. After immobilization the immobilization cups and excess of ligand need to be removed to reattain a flat analysis surface.

On chip droplet manipulation, to address ligands to specific positions of the sensor area, followed by photo-activation and immobilization could facilitate another valid alternative to the existing techniques and avoid drying of the ligands during the immobilization process.

\subsubsection{Increased measurement capacity and throughput}

The $7 \times 7 \mathrm{~mm}$ measurement area of the SPR imager should be used more efficiently, now only $3.5 \times 2.5 \mathrm{~mm}$ is used. In theory the surface area can contain 4 microarrays made with the spot-24 printhead of the TopSpot. A microarray produced by the spot-96 printhead would fit in the flow-cell, and also increases the throughput compared to the spot-24 printhead. An increase of the illuminated surface area, would provide a larger measurement area and thereby facilitating more spots.

The use of parallel flow-cells in combination with microarrays also increases the throughput as the samples can be injected simultaneously.

Adjustment of the sample injection system in combination with a more complex sample chip facilitating switching between the interaction cycle steps, would decrease the interaction times and thereby lead to higher throughput of samples.

Adaptations in liquid handling system and flow-cell can lead so smaller sample volume, however, this cannot be done without limitations. E.g. depletion of the analyte in the sample plug may occur.

\subsubsection{PDMS stop-valves}

Other applications for the PDMS stop-valves need to be further investigated, such as contact angle measurement, fluid viscosity measurement and Lab-on-a-Chip based 
applications like pre-filling of channels with fluids containing chemical or biological components.

\subsubsection{Kinetic modeling}

A more detailed study to prove the general applicability of the ligand immobilization based assay for kinetic analysis, by testing other model systems besides the biotinylated peptides in ratio- and buffer-dilution is needed. The possibility of more general dilution molecules that can be used for the ratio-dilution has to be investigated, as it is not feasible to always get the same molecule without the epitope for the ratio dilution, and moreover they are too costly. Another advantage of the ratio-dilution is the reduction of the amount of spots which are necessary in a microarray for e.g. concentration and kinetic studies. For a concentration study the ligand density should be as high as possible while for kinetic studies the ligand density should be low to improve the monovalent behavior of the bivalent molecules. To find out how much the ligand density and organization at the surface correlates to the binding behavior of the molecules, and till what extent this can be predicted, the homogeneity of the surface immobilization could be studied in more detail by e.g. fluorescence microscopy, scanning electron microscopy or atomic force microscopy. The controlled ligand density is not only important for kinetic analysis, other assays can also benefit from this new assay protocol. In buffer diluted ligand immobilization procedure the saturation effect as shown in chapter 6 occurs and therefore more spots should be applied for the ligand series. Therefore if ligands are diluted in ratio with a similar non interacting molecule only a few spots are needed for a high to low series coupling of the ligand to the surface. In this way a microarray can be fabricated more efficiently for both kinetic modeling and concentration measurements.

Analysis of the data obtained with other kinetic models and software available can provide valuable information of the general applicability of the newly proposed assay protocol. The possibility to analyze SPR data obtained from different systems in a general software e.g. Scrubber, is convenient and moreover encourages scientists to use it because it facilitates direct comparison of the various available systems.

The effect of fluorescent and other labels to the binding properties of molecules needs to be further investigated as a pilot study showed a decreased affinity caused by labeling, 
shown in Fig.7.1. This can be an additional stimulus for the scientific community to further pursue the development of sensitive label-free detection methods.

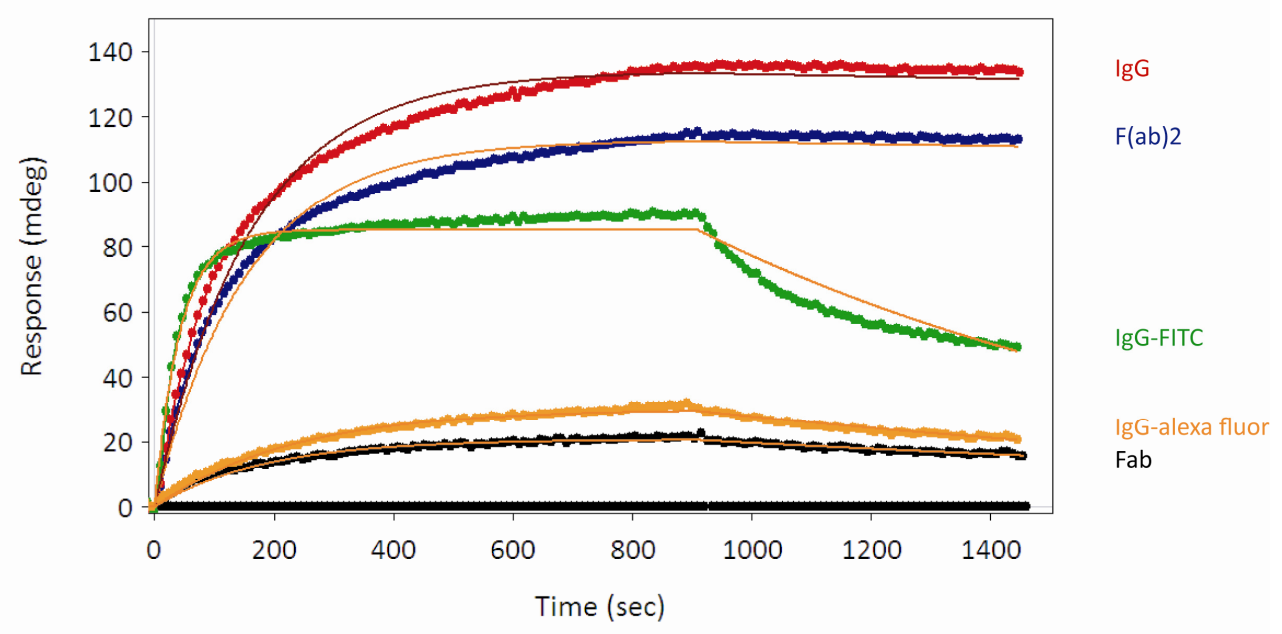

Fig. 7.1. Although the concentration of the analytes are diluted to $25 \mathrm{nM}$, a clear difference of concentration (initial slope) and kinetics can be observed. The IgG-FITC and IgG-alexa fluor show a lower affinity (higher dissociation rate, which could be affected by the label) compared to the unlabeled IgG.

\subsubsection{SPR imaging hardware and software}

If the ROI's could become smaller by increasing the resolution of the CCD camera to a pixel size of $1 \times 1 \mu \mathrm{m}$ or less, compared to the current pixel size is $7.5 \times 7.5 \mu \mathrm{m}$, the measurable microarray spots could become smaller too. When decreasing the measurement spot or ROI and analyte volumes, the sensitivity of the measurement may need to be optimized by e.g. light source, detector sensitivity or signal amplification.

The analysis software SPRINT needs to be adapted for analysis of both, the analyte and ligand based assay. Improvement of the software with a user friendly interface also increases the throughput. An implementation of full kinetic analysis would be an advantage, not to have to import the data into Scrubber, because this is a time consuming process and the software is not designed for ligand based kinetic analysis. 



\section{Summary}

This thesis, "Biomolecular interaction sensing on microarrays using surface plasmon resonance imaging", gives a brief introduction to biomolecular interactions in general. And includes interactions of peptides and proteins, antibodies and autoimmune diseases. Furhtermore, the concept of label-free biosensing is introduced, with a special emphasis on SPR imaging. The principle and role of microarrays and data analysis are also described.

Chapter 3 explains the soft lithography process to develop various types of PDMS spotting devices for the immobilization of proteins in confined surface areas in a microarray format. PDMS was explored to provide a disposable alternative for the TopSpot, however, in combination with our experimental restrictions the PDMS based method did not succeed and we continued using the Topspot.

In chapter 4 and 5 the use of a scanning-angle surface plasmon resonance (SPR) imaging instrument for monitoring the binding of biomolecules on user-defined regions of interest of a microarray is described. Peptides and proteins were both spotted on the same sensor chip to illustrate that both, low and high molecular weight ligands with initial large differences in off-set SPR angles, can be applied within the same experiment. The effectiveness of this system is demonstrated by automatically monitoring the interactions between citrullinated peptides and serum autoantibodies of 50 rheumatoid arthritis (RA) patients and 29 controls in a single step.

Chapter 6 deals with a new assay protocol based on injecting a single analyte concentration, and exposing it to spots with various ligand densities, rather than multiple analyte injections of varying analyte concentrations exposed to a single ligand surface, as is done in the conventional procedure is proposed. The new method uses controlled dilution of ligands with background molecules which, facilitates immobilization of a precise ligand density on the surface, which is a prerequisite for direct kinetic analysis. This alternative to the conventional multi-analyte overlay plot for calculating the rate- and affinity constants of a biomolecular interaction, has the advantage of requiring only a single analyte injection, fewer surface regeneration steps, and a reduction of the overall assay time. As a model system, various biotin specific interactions have been tested by means of SPR imaging for their affinity toward surface immobilized biotinylated peptides. 


\section{Samenvatting}

Dit proefschrift, "Biomolecular interaction sensing on microarrays using surface plasmon resonance imaging", geeft een korte en algemene introductie van biomoleculaire interacties. Het omvat onder andere de interacties van eiwitten en peptiden, antilichamen en autoimmuun ziektes. Verder wordt het concept van labelvrije biosensing geïntroduceerd, waarbij extra nadruk wordt gelegd op SPR imaging. Het principe en het belang van microarrays en de daarbij behorende data analyse wordt ook beschreven.

Hoofdstuk 3 beschrijft het soft-lithografische proces om PDMS spot apparaatjes ten behoeve van eiwit immobilizatie op begrensde oppervlakken in een microarray formaat te maken. Er is gekeken of PDMS een wegwerpbaar alternatief kan vormen voor de TopSpot. Maar in combinatie met de experimentele beperkingen bleek de op PDMS gebaseerde methode niet succesvol, en daarom is besloten verder te gaan met de TopSpot.

Het gebruik van het hoekscannende SPR imaging instrument voor het meten van biomoleculaire binding op zelf gedefinieerde regionen van interesse, word beschreven in de hoofdstukken 4 en 5 . Om aan te tonen dat liganden met zowel hoge als lage moleculaire massa en grote verschillen in de initiële SPR hoek offset tegelijk gemeten kunnen worden, zijn eiwitten en peptiden op dezelfde sensor chip gespot. De effectiviteit van het systeem is gedemonstreerd door middel van het geautomatiseerd meten van de interactie van gecitrullineerde peptiden en serum autoantilichamen van 50 reumatoïde artritis patiënten en 29 contoles.

Hoofdstuk 6 behandeld een nieuwe meetmethode gebaseerd op de injectie van een enkele analyte concentratie om deze vervolgens bloot te stellen aan verschillende ligand concentraties. Dit in tegenstelling tot de gebruikelijke methode waarbij verschillende analyte concentraties worden geïnjecteerd op een enkele ligand concentratie. De nieuwe methode maakt gebruik van een gecontroleerde verdunning van de liganden op basis van achtergrond moleculen. Hierdoor kan de ligand concentratie nauwkeurig kan worden gecontroleerd, wat een vereiste is voor kinetische bepalingen. Deze alternatieve methode voor het maken van overleg grafiek voor de berekening van snelheid en affiniteit constanten, heeft als grote voordeel dat een enkele analyte concentratie volstaat. Hierdoor zijn minder regeneratie stappen nodig en wordt de totale meettijd gereduceerd. Verschillende biotine specifieke interacties zijn gemeten en gebruikt als model systeem voor de affiniteits metingen met het SPR imaging instrument. 


\section{List of abbreviations}

AFM

APTS

$\mathrm{BS}^{3}$

CCD

CDNA

CE

DNA

DPN

EDC

ELISA

Fab

$\mathrm{F}(\mathrm{ab})_{2}$

FFE

FITC

FLIP

FRAP

GC-SPR

$\mathrm{HC} 80 \mathrm{~m}$

HTS

IEF

IgG

iSPR

$\mathrm{k}_{\mathrm{a}}$

$k_{d}$

$K_{D}$ atomic force microscopy

8-aminopyrene-1,3,6-trisulfonic acid trisodium salt

suberic acid bis(3-sulfo-N-hydroxysuccinimide ester) sodium salt charged-coupled device complementary deoxyribonucleic acid capillary electrophoresis deoxyribonucleic acid dip pen nanolithography 1-Ethyl-3-(3-dimethylaminopropyl) carbodiimide enzyme linked immunosorbent assay fragment of antibody, monovalent fragment of antibody, bivalent free-flow electrophoresis fluorescein isothiocyanate fluorescence loss in photobleaching fluorescence recovery after photobleaching grating-coupled surface plasmon resonance carboxylated hydrogel with $80 \mathrm{~nm}$ thickness high-throughput screening isoelectric focussing immunoglobulin gamma imaging surface plasmon resonance association rate constant dissociation rate constant dissociation constant 


\begin{tabular}{|c|c|}
\hline kDa & kilo Dalton \\
\hline LOC & lab-on-a-chip \\
\hline LOD & limit of detection \\
\hline MES & morpholinoethane sulfonic acid \\
\hline MS & mass spectrometry \\
\hline NHS & $N$-hydroxysuccinimide \\
\hline PAGE & polyacrylamide gel electrophoresis \\
\hline PBS & phosphate buffered saline \\
\hline PBST & phosphate buffered saline / tween 20 \\
\hline PDMS & polydimethylsiloxane \\
\hline PEG & polyethylene glycol \\
\hline $\mathrm{pH}$ & power of hydrogen \\
\hline PI & isoelectric point \\
\hline RA & rheumatoid arthritis \\
\hline RIA & radioimmunoassay \\
\hline RNA & ribonucleic acid \\
\hline ROI & region of interest \\
\hline SDS & sodium dodecyl sulfate \\
\hline SECM & scanning electrochemical microscopy \\
\hline SPFS & surface plasmon field-enhanced fluorescence spectroscopy \\
\hline SPR & surface plasmon resonance \\
\hline ssDNA & single stranded deoxyribonucleic acid \\
\hline SU-8 & negative photoresist \\
\hline WC-SPR & waveguide-coupled surface plasmon resosnace \\
\hline 2-D & two dimensions \\
\hline
\end{tabular}




\section{List of publications}

\section{Papers}

J.B. Beusink, G. Krishnamoorthy, E.T. Carlen, A. van den Berg, R.B.M. Schasfoort. Ratiodiluted ligand density assay for single analyte kinetic analysis. Manuscript in preparation.

J.B. Beusink, A.M.C. Lokate, G.A.J. Besselink, G.A.J. Pruijn, R.B.M. Schasfoort. Anglescanning SPR imaging for detection of biomolecular interactions on microarray. Biosensors and Bioelectronics. 2008, 23, 839-844.

A.M.C. Lokate, J.B. Beusink, G.A.J. Besselink, G.A.J. Pruijn, R.B.M. Schasfoort. One-step multiplex biomolecular interaction monitoring by scanning SPR imaging. Journal of the American Chemical Society. 2007, 129, 14013-14018.

\section{Book contributions}

R.B.M. Schasfoort, A.M.C. Lokate, J.B. Beusink, G.J.M. Pruijn, G.H.M. Engbers. Measurement of the analysis cycle: Scanning SPR microarray imaging of autoimmune diseases. Handbook of surface plasmon resonance. 2008, RSC publishing, ISBN: 978-085404-267-8.

G. Krishnamoorthy, J.B. Beusink, E.T. Carlen, S. Schlautmann, A. van den Berg, R.B.M. Schasfoort (2007, October 9). Integrated Lab-on-a-Chip and SPR imaging for label-free microarray biosensing. Paris, France, $\mu$ TAS 2007, the $11^{\text {th }}$ international conference on miniaturized systems for chemistry and life sciences. ISBN:978-0-9798064-0-7 (2 volume set).

J.B. Beusink, R.B.M. Schasfoort. Genomics 2030 part of everyday life. 2005, STT/Beweton, The Hague, The Netherlands. ISBN: 90-809613-2-9. 


\section{Oral presentations}

J.B. Beusink, R.B.M. Schasfoort (2007, October, 24). Label free biosensing; kinetics of multiple biomolecular interactions. Leuven, Belgium, Frontiers annual meeting

J.B. Beusink, R.B.M. Schasfoort (2007, June, 6). Autoantibody detection in serum samples by SPR imaging. Edmonton, Canada, visited the group of Marc McDermott.

J.B. Beusink, A.M.C. Lokate, G.A.J. Besselink, G.J.M. Pruijn, R.B.M. Schasfoort (2007, March 21). Autoantibody detection in serum samples by SPR imaging. Zermatt, Switzerland, workshop 'Exploring new frontiers in BIO/NANO'.

J.B. Beusink, A.M.C. Lokate, G.A.J. Besselink, G.J.M. Pruijn, R.B.M. Schasfoort (2006, November 15). Automated SPR imaging of peptide microarrays for detection of Rheumatoid Arthritis autoantibodies. Montreux, Switzerland, NanoTech2006- $10^{\text {th }}$ Annual European Conference on Micro \& Nanoscale Technologies for the Biosciences.

J.B. Beusink, G.A.J. Besselink, R.B.M. Schasfoort (2006, October 25). SPR imaging for protein microarray analysis. Sicily, Italy, Frontiers annual meeting.

J.B. Beusink, G.A.J. Besselink, R.B.M. Schasfoort (2006, September 28). Antibody binding to protein microarrays using fluorescence microscopy and SPR imaging. Enschede, The Netherlands, MESA+ annual meeting.

J.B. Beusink, G.A.J. Besselink, R.B.M. Schasfoort (2004, September 30). Development of a protein array using a microfluidic spotting device. Enschede, The Netherlands, MESA+ annual meeting. 


\section{Posters}

S. Chen, M. Jin, G. Krishnamoorthy, J.B. Beusink, M.B.M. Harink, M. Nasir, E.T. Carlen, A. van den Berg (2008, September 23). Label-free nanobiosensing. Enschede, The Netherlands, MESA+ annual meeting.

J.B. Beusink, G. Krishnamoorthy, A.M.C. Lokate, R.B.M. Schasfoort, A. van den Berg (2008, April 7-9) SPR imaging - biomolecular interactions without a trace. Boekelo, The Netherlands, LFB2008, first label free biosensing meeting.

J.B. Beusink, G. Krishnamoorthy, A.M.C. Lokate, R.B.M. Schasfoort, A.van den Berg (2007, September 11) SPR imaging - biomolecular interactions without a trace. Leuven, Belgium, Frontiers annual meeting.

G. Krishnamoorthy, J.B. Beusink, E.T. Carlen, S. Schlautmann, A. van den Berg, R.B.M. Schasfoort (2007, October 9). Integrated Lab-on-a-Chip and SPR imaging for label-free microarray biosensing. Paris, France, $\mu$ TAS 2007, the $11^{\text {th }}$ international conference on miniaturized systems for chemistry and life sciences.

J.B. Beusink, G. Krishnamoorthy, A.M.C. Lokate, R.B.M. Schasfoort, A.van den Berg (2007, September 11) SPR imaging - biomolecular interactions without a trace. Enschede, The Netherlands, MESA+ annual meeting.

A.M.C. Lokate, J.B. Beusink, G.A.J. Besselink, G.A.J. Pruijn, R.B.M. Schasfoort (2006, November 8). Automated SPR imaging of peptide microarrays for multi-analyte detection of Rheumatoid Arthritis. Enschede, The Netherlands, Dutch forum.

J.B. Beusink, G.A.J. Besselink, R.B.M. Schasfoort (2006, November 2). SPR imaging for protein microarrays for detection of antibody binding. Amsterdam, The Netherlands, AMT$2^{\text {nd }}$ annual meeting in Advances in Microarray Technologies. 
J.B. Beusink, G.A.J. Besselink, R.B.M. Schasfoort (2006, September 28). SPR imaging for protein microarrays for detection of antibody binding. Enschede, The Netherlands, MESA+ annual meeting.

J.B. Beusink, G.A.J. Besselink, A.J. Tüdős, R.B.M. Schasfoort (2005, November 14-16). A novel PDMS based approach for the fabrication of protein microarrays. Montreux, Switzerland, NanoTech2005- $9^{\text {th }}$ Annual European Conference on Micro \& Nanoscale Technologies for the Biosciences.

R. Verdoold, M.B.M. Harink, J.B. Beusink, G.A.J. Besselink, R.B.M. Schasfoort (2005, March 31- April 1). Techniques for creating microarrays for Surface Plasmon Resonance-based protein assays. Wageningen, The Netherlands, Sense of contact. 


\section{Acknowledgements}

Nearly 5 years ago I started working in the Biochip group, a small but very dynamic group. The switch from molecular genetics to biosensor technology, and having to deal with physicist rather than biologist was much more challenging than I expected. Nevertheless, I managed to turn the initially blank pages of this book into a colorful representation of what kept me busy these years.

As a scientist you are not on your own, you are part of a lab inhabited by many other scientists. It is like in nature, a good organization is in symbiosis. This part of my thesis is devoted to convey my gratitude to those who helped me, MANY THANKS TO YOU ALL!!!! Sure I could stop writing now, however, I would like to thank some of you in person.

Richard Schasfoort, for giving me this great opportunity of scientific and personal growth and believing in me and my project. Over the years I learned to appreciate the iSPR, luckily it was too heavy during the days I did not. After a thorough investigation I came to the conclusion it is "Bianca-proof", and I wish you all the best in making it into a great success. I want to thank all the present and past members of the Biochip group for their help and moral support over the years. You created a very nice working atmosphere, also outside the working hours when we turned into the "Beerchip group". Many thanks for all the good times spend in the Penthouse and downtown with your great company!

Hans de Boer, without your listening ear I would have not survived. Discussing problems always led to interesting and ingenious solutions. Stefan Schlautmann, the PDMS work would have not been the same without you. Thanks for the help in designing and making the masks. Geert Besselink, you introduced me to the iSPR during your stay at IBIS. The one person who did not fear proteins, thanks for supervising me. Anna Tudos, shortly I was not the only women in the Biochip group and your view of the scientific world around me was very refreshing.

The Biochip-Phd students: Dietrich Kohlheyer, your amazing movies and animations often scared me- "Would I ever be able to impress anybody with my results?" Thanks to you I also managed to have a cool movie! Dawid Zalewski, you proved there are more sides of a person. You always managed to make me smile using one of your funny faces or actions. Arnoud Thijssen, the time we shared the office was short but simply great and filled with many interesting discussions about the various topics of life. Ganeshram Krishnamoorthy, 
the last year we shared the office. I learned a lot from your patience in analyzing the kinetics data, thanks for showing me how to do it. And good luck finishing your PhD. Angelique Segbers, officially you were stationed in Nijmegen but in the end we spend quite some time together in the lab in Enschede. Thanks for all the good times and the work we managed to accomplish together.

The Biochip-students: Verena Stimberg, the many laughs we had in the lab drove some group members crazy. But the road-trip we made through the Canadian Rockies' was absolutely unforgettable, many thanks! Björn Harink, over the years you proved to be much more serious and sensitive than I expected. Thanks for all the good times and conversations, and good luck with your PhD. Remco Verdoold, you had so many amazing stories to tell. Sandeep Unnikrishnan, you introduced me to the rich and colorful culture of India.

Vinod Subramaniam, you and your group provided an amazing scientific environment in your new lab facility in the Zuidhorst. Many thank for all the understanding and support. Sylvia Winters, you are so much more than just a secretary! You made me feel very welcome and I always enjoyed the non-scientific breaks we had together. All the BPE group members, thanks for the hospitality and the time spend working together and during the social gatherings.

Albert van den Berg, I really enjoyed the work discussions we had. Although I felt like an outsider in your lab-on-a-chip group, you made me feel welcome and provided the right supervision I needed to finish my thesis. Edwin Carlen, I really enjoyed your interesting sense of humor. Many thanks for your critical questions and help with my writing, due to you I managed to improve the quality of my thesis a lot! Eddy de Weerd, we had many discussions about how to manufacture PDMS devices in an easy way. The chocolates combined with good company always made it worth to step in your office. Severine le Gac, our trip by night train to Zermatt was so much fun. Erik Staijen, I really enjoyed your company and all the good conversations and laughs we had. I wish you all the best with B4G! Rogier Veenhuis, it was so nice to find someone trying to finish and working late just like me. Thanks for always being in a good mood! And all the other BIOS members, Ad, Arpita, Bertjan, Catlijn, Daniel, Egbert, Erik F, Evelien, Floor, Georgette, Hermine, Iris, Jan E, Jan vN, Johan, Lingling, Loes, Mathieu, Mingliang, Muhammad, Paul, Rerngchai, Robertjan, Songyue, Wouter $\mathrm{O}$, Wouter $\mathrm{S}$, thanks for the help and the good times. 
Then I have to thank my friends who have helped me through these challenging years in various ways. By listening to me, helping me or just being there to bring me back with both my feet on the solid ground. Juliane Teapal, we met as you were a master student in the BIOS group. Soon we realized we had much more in common then we imagined. Thanks for all the fun and good times. But moreover for listening to all the things I had to say, and supporting me the way I needed it. I really liked talking over a nice coffee in the morning. Thanks for being such a good friend! Mink Hoexum, I really enjoyed our dinners together. You made me relax and enjoy good company in such a peaceful manner. Unfortunately we did not have enough time during the last year to see each other as often as before. But no matter where you are, you are on my mind. Mireille van Damme en Marjolein Wildwater, we met while being colleagues in Utrecht and you have been my friends from A'dam. I really missed having you around on a daily basis during the first years of my Phd. Therefore I really enjoyed all the times when we met up for tapas and rose in the Jordaan. I look forward to see us, the three musketiers, going for tapas together again. Miss you girls! Sandra van Kanten, you made me become more sportive by dragging me to the gym. I always enjoyed the good conversations we had together, also with you Herman. Agnes Klooster, we go back quite some time as we already met without knowing it - when we were baptized. Thanks you for having been there always, and for all the nice weekends we spend together these last years. What is better than de-stressing with good company! Thanks for the support in the next step in my life and being such an amazing friend!

Last but not least my family. My little bro, Jan Willem. The many times you took care of Noa, Kuma and Eek made me able to travel around quite a bit. I really enjoyed the salsa classes we took together combined with those nice dinners. Thanks for all the support and good times spend together. I'll be there for you, as you have been there for me too! My little sister, Jolanda. Who is no longer little and already has a wonderful family of her own, her husband Jeroen en son Dim. I'm so proud of you! Thanks for all the support during the past years. To my grandparents, I'm finally done studying but l'll never be done learning. Thank you mom and dad for supporting me and listening to the many frustrations I had to deal with over the years. You gave me the freedom to make my own decisions in life. Without your love and support I would not have been the person I am today! I love you!

Bianca. 
